\title{
Translating Xd-C Programs to MSVL Programs
}

\author{
Meng Wang, Cong Tian*, Nan Zhang*, Zhenhua Duan*, Chenguang Yao \\ ICTT and ISN Lab, Xidian University, Xi'an, 710071, China
}

\begin{abstract}
$\mathrm{C}$ language is one of the most popular languages for software systems. In order to verify safety, reliability and security properties of such systems written in $\mathrm{C}$, a tool UMC4M for runtime verification at code level based on Modeling, Simulation and Verification Language (MSVL) and its compiler MC is employed. To do so, a C program $P$ has to be translated to an MSVL program $M$ and the negation of a desired property $Q$ is also translated to an MSVL program M', then "M and M" is compiled and executed armed with MC. Whether $P$ violates $Q$ is checked by evaluating whether there exists an acceptable execution of new MSVL program " $M$ and $M$ "." Therefore, how to translate a $C$ program to an MSVL program is a critical issue. However, in general, $\mathrm{C}$ is of complicated structures with goto statement. In this paper, we confine the syntax of $\mathrm{C}$ in a suitable subset called $\mathrm{Xd}-\mathrm{C}$ without loss of expressiveness. Further, we present a translation algorithm from an Xd-C program to an MSVL program based on translation algorithms for expressions and statements. Moreover, the equivalences between expressions and statements involved in Xd-C and MSVL programs are inductively proved. Subsequently, the equivalence between the original Xd-C program and the translated MSVL program is also proved. In addition, the proposed approach has been implemented by a tool called $C 2 M$. A benchmark of experiments including 13 real-world Xd-C programs is conducted. The results show that $C 2 M$ works effectively.
\end{abstract}

Keywords: C language, MSVL, translation, model checking, verification

\section{Introduction}

Software systems written in C language are more than 13\% 1, second popular one, in the world since $\mathrm{C}$ can be used to implement a complex system in a flexible way. In order to verify properties of safety, reliability and security of such systems, many researchers focus on model checking [1, 2]. For the

\footnotetext{
*Corresponding authors: Cong Tian (ctian@mail.xidian.edu.cn), Nan Zhang (nanzhang@xidian.edu.cn) and Zhenhua Duan (zhhduan@mail.xidian.edu.cn). This research is supported by the National Natural Science Foundation of China under Grant Nos. 61420106004,61732013 and 61751207.

${ }^{1}$ https://www.tiobe.com/tiobe-index/
} 
purpose of employing conventional model checkers such as NuSMV [3] and SPIN [4, an abstract model has to be extracted from a $\mathrm{C}$ program, and a desired property is specified by an LTL [5] or CTL [6] formula. Then the model checkers check whether the abstract model satisfying the property is valid. However, as software systems become larger and more complex, it is difficult to acquire a model which is consistent with the original program.

In recent years, verifying software systems at code level has attracted more attentions [7, 8, 9, 10, 11, 12]. Tools like, SLAM [13, BLAST [14, CPAChecker [15] and CBMC [16], support only safety property verification. They insert assertions into $\mathrm{C}$ source code and then the verification is carried out by checking the reachability of error labels. In order to verify more temporal properties such as liveness, Ultimate LTLAutomizer [12] and T2 [17 extend the software model checking approach by reducing the verification problem to fair termination checking. To do that, a program to be verified written in $\mathrm{C}$ is first translated to an intermediate form, and then a desired property can be verified based on automata-theoretic approach for verification. However, all these tools suffer from the state-explosion problem. Further, since there are no execution details of programs, the verification result is not always accurate, that is, sometimes false positives (i.e., potential errors may be reported where there are none) or false negatives (i.e., errors are not reported) may be produced.

As a lightweight verification technique, runtime verification checks whether a run of a system to be verified satisfies a given property by monitoring the execution of the system. It alleviates the state-explosion problem since a single execution path is checked each time. With this approach, a system to be verified is described in a conventional program while a desired property is expressed in a formal specification language. Therefore, extracting events from the executing system and sending them to monitors can generate a large runtime overhead. A better solution is to implement the system and monitor within the same logical framework. The runtime verification tool UMC4M [18, 19] takes a program $M$ written in a Modeling, Simulation and Verification Language (MSVL) [20, 21, 22, 23] and a desired property $P$ specified by a Propositional Projection Temporal Logic (PPTL) [24, 25] formula as input, and converts temporal property verification as a dynamic program execution task. With this tool, the negation of the desired property is translated to an MSVL program M', then whether $\mathrm{M}$ violates $P$ is checked by evaluating whether there exists an acceptable execution of new MSVL program "M and M" ". Therefore, verification of MSVL programs can be carried out in the same logical framework.

In order to verify $\mathrm{C}$ programs by means of UMC4M, they have to be rewritten to MSVL programs. In this paper, we confine the syntax of $\mathrm{C}$ language in a suitable subset called $\mathrm{Xd}-\mathrm{C}$ and propose an algorithm to automatically translate $\mathrm{Xd}-\mathrm{C}$ programs to MSVL programs. Xd-C features most of the data types and statements of $\mathrm{C}$, including all arithmetic types, arrays, pointers and struct types, and all statements except goto statement, while MSVL data types and statements include all Xd-C data types and statements. Therefore, an Xd-C program can be translated to an equivalent MSVL program in an automatic way. In fact, we can treat that all variables in the MSVL program are framed, and the trans- 
lation is in one-to-one manner. The time complexity of the translation is linear $(O(n))$, where $n$ is the number of statements in an $\mathrm{Xd}-\mathrm{C}$ program. To prove the equivalence between the original Xd-C and translated MSVL programs, we present the operational semantics of Xd-C and MSVL. The operational semantics of Xd-C is similar to that of another subset of $\mathrm{C}$ language proposed by Blazy et al., called Clight [26]. It is presented as a big-step operational semantics and characterizes both termination and divergence behaviors. Whereas in MSVL, the evaluation rules for left-value and right-value arithmetic expressions, and Boolean expressions are borrowed from [27]. The semantic equivalence rules regarding a program, the transition rules within a state and interval transition rules are also formalized [22, 27, 28]. Further, based on operational semantics of Xd-C and MSVL, we prove equivalences between expressions and statements by means of structural induction and rule induction, respectively.

The contributions of this paper are three-fold:

(1) We present algorithms to translate declarations, expressions and statements from Xd-C to MSVL. An example bzip2 [29, a compression program to compress and decompress input files, is used to show how the algorithms work.

(2) The equivalence between an original $\mathrm{Xd}-\mathrm{C}$ program and the translated MSVL program is proved based on operational semantics of $\mathrm{Xd}-\mathrm{C}$ and MSVL.

(3) We have implemented a translator $C 2 M$ and conducted a benchmark of experiments including 13 real-world Xd-C programs.

The remainder of this paper is organized as follows. In Section 2, $\mathrm{Xd}-\mathrm{C}$ is briefly introduced. Further, MSVL is introduced in Section 3 . Section 4 presents an algorithm for translating Xd-C programs to MSVL programs. Subsequently, in Section 5 , the equivalences between expressions and statements in $\mathrm{Xd}-\mathrm{C}$ and MSVL are proved, respectively. Moreover, the equivalence between Xd-C and translated MSVL programs is also proved. An implementation of the proposed approach is presented and the evaluation is conducted in Section 6. Section 7 concludes the paper.

\section{The Restricted C Fragment: Xd-C}

The restricted $\mathrm{C}$ fragment called $\mathrm{Xd}-\mathrm{C}$ is confined in a subset of ANSI-C (C89 standard version). It consists of often-used types, expressions and statements of $\mathrm{C}$ language. $\mathrm{Xd}-\mathrm{C}$ is similar to Clight [26] but more than Clight.

\subsection{Types}

The supported types in Xd-C include arithmetic types (char, int, float and double in various sizes and signedness), pointer, void pointer, function pointer and struct types. However, union type, static local variables and type qualifiers such as const, restrict and volatile are not allowed in Xd-C. As storage-class 
specifiers, typedef definitions have been expanded away during parsing and typechecking. The syntax of $\mathrm{Xd}-\mathrm{C}$ types is given as follows:

$$
\begin{array}{lrl}
\text { Signedness } & \text { sign } & := \\
\text { int length } & \text { len } & :=\text { short } \mid \text { lonsigned } \\
\text { Types } & \tau & :=\text { int } \mid \text { sign int } \mid \text { len int } \mid \text { sign len int } \\
& & \mid \text { float } \mid \text { double } \mid \text { long double } \mid \text { char } \mid \text { sign char } \\
& & \mid \text { structself } \mid \text { voidp } \mid \text { functp } \mid \tau *
\end{array}
$$

Self-defined Types:

$$
\begin{array}{ll}
\text { structself } & ::=\text { struct } i d_{1}\left\{\left(\tau \text { id } d_{2} ;\right)^{+}\right\} \mid \text {struct } i d_{1} \\
\text { functp } & ::=[\tau \mid \text { void }]\left((\tau,)^{*} \tau\right) * \mid[\tau \mid \text { void }]() * \\
\text { voidp } & ::=\text { void } *
\end{array}
$$

where struct $i d_{1}\left\{\left(\tau i d_{2} ;\right)^{+}\right\}$defines a structure $i d_{1}$ consisting of body $\left(\tau i d_{2} ;\right)^{+}$; $[\tau \mid$ void $]\left((\tau,)^{*} \tau\right) *$ defines a function pointer with each parameter of type $\tau$ and a return value of type $\tau$ or void. $[\tau \mid$ void $]() *$ defines a function pointer with no parameter. Note that $i d$ (possibly with subscriptions) is a string (name) consisting of characters and digits with a character as its head.

\subsection{Expressions}

The expression $e$ in $\mathrm{Xd}-\mathrm{C}$ is inductively defined as follows:

$$
\begin{aligned}
& e::=c|l e| \& l e|(\tau) e| x\left(e_{1}, \ldots, e_{k}\right)\left|o p_{1} e\right| e_{1} o p_{2} e_{2} \mid e_{1} e_{2}: e_{3} \\
& x::=\quad i d|i d[e]| i d\left[e_{1}\right]\left[e_{2}\right]|l e . x| l e \rightarrow x \\
& \text { le }::=x \mid * e \\
& \text { op } 1::=+|-|^{\sim} \mid \text { ! } \quad \text { op } 2::=\text { aop } \mid \text { bop } \mid \text { rop } \mid \text { eop } \mid \text { lop } \\
& \text { aор }::=+|-| *|/| \% \quad \text { bop }::=\quad<<\left.|>>| \&||\right|^{\wedge} \\
& \text { rop }::=\quad \text { eop }::=|<=|>==\mid != \\
& \text { lop }:=\& \& \mid \|
\end{aligned}
$$

where $c$ is an arbitrary constant, $i d$ a variable, $i d[e]$ the eth element of array $i d$ (counting from 0$), i d\left[e_{1}\right]\left[e_{2}\right]$ the element in row $e_{1}$ and column $e_{2}$, le.x member $x$ of structural variable $l e$ and $l e \rightarrow x$ member $x$ of the structural variable that le points to. \&le takes the address of $l e$ and $* e$ is the pointer dereferencing. $x\left(e_{1}, \ldots, e_{k}\right)$ is a function call with arguments $e_{1}, \ldots, e_{k}$ and it does not change the memory state. $(\tau)$ e represents the type cast of $e$ namely converting the value of $e$ to the value in type $\tau$. The type of $e$ before the type cast is a nonpointer type. $o p_{1} e$ is a unary expression including $+e,-e, \sim e$ and !e. $o p_{2}$ represents an binary operator including arithmetic operators aop $(+,-, *, /$ and $\%)$, bitwise operators bop $\left(<<,>>, \&, \mid\right.$ and $\left.{ }^{\wedge}\right)$, relational operators rop $(<,>,<=$ and $>=)$, equality operators eop $(==$ and $!=)$ and logical operators lop $(\& \&$ and $॥)$. Both $e_{1}$ and $e_{2}$ in $e_{1}$ rop $e_{2}$ are of non-pointer types. $e_{1} ? e_{2}: e_{3}$ is a conditional expression indicating that the result is $e_{2}$ if $e_{1}$ is not equal to 0 , and $e_{3}$ otherwise. 


\subsection{Statements}

The following are the elementary statements in Xd-C:

\begin{tabular}{|c|c|c|}
\hline \multirow[t]{11}{*}{ Statements: } & $c s::=$ & null \\
\hline & $\mid l e++$; & post increment \\
\hline & le---; & post decrement \\
\hline & $l e=e$ & assignment \\
\hline & $\operatorname{if}(e)\left\{c s_{1}\right\}$ else $\left\{c s_{2}\right\}$ & conditional \\
\hline & $\mid \operatorname{switch}(e)\{s w\}$ & switch \\
\hline & $\mid$ while $(e)\{c s\}$ & while loop \\
\hline & do $\{c s\}$ while $(e)$ & do loop \\
\hline & $\mid$ for $\left(c s_{1} ; e ; c s_{2}\right)\{c s\}$ & for loop \\
\hline & $\begin{array}{l}\text { |continue; } \\
\text { |break; }\end{array}$ & $\begin{array}{l}\text { next iteration of the current loop } \\
\text { exit from the current loop }\end{array}$ \\
\hline & $\begin{array}{l}\text { |return } e \\
\text { |return; }\end{array}$ & return from the current function \\
\hline \multirow{5}{*}{ Switch cases: } & $\mid c s_{1} ; c s_{2}$ & sequence \\
\hline & $x\left(e_{1}, \ldots, e_{k}\right)$ & function call \\
\hline & $s w::=s w_{1} \mid s w_{2} ; s w_{1}$ & \\
\hline & $s w_{1}::=$ default $: c s$ & default case \\
\hline & $s w_{2}::=$ case $n: c s ; s w_{2}$ & labeled case \\
\hline
\end{tabular}

A null statement performs no operations. A post increment statement le++ means that the value of $l e$ is changed to $l e+1$ while a post decrement statement $l e--$ indicates that the value of $l e$ is changed to $l e-1$. In an assignment statement "le $=e ;$ ", the value of $e$ replaces the value stored in the location designated by $l e$. In a conditional statement if $(e)\left\{c s_{1}\right\}$ else $\left\{c s_{2}\right\}, c s_{1}$ is executed if expression $e$ compares unequal to 0 , and $c s_{2}$ is executed otherwise. In a switch statement $\operatorname{switch}(e)\{s w\}, e$ is the controlling expression, and the expression of each case label shall be an integer constant expression. There are three kinds of iteration statements in Xd-C including while loop, do loop and for loop statements. An iteration statement causes the body of the loop to repeatedly execute until controlling expression $e$ equals 0 . In a while loop statement while $(e)\{c s\}$, the evaluation of $e$ takes place before each execution of $c s$ while in a do loop statement "do $\{c s\}$ while $(e)$;", the evaluation of $e$ takes place after each execution of $c s$. In a for loop statement for $\left(c s_{1} ; e ; c s_{2}\right)\{c s\}, c s_{1}$ executes once at the beginning of the first iteration, $e$ is the condition of the loop, $c s_{2}$ executes at the end of each iteration, and $c s$ is the body of the loop. Jump statements including "continue;", "break;", "return e;" and "return;" are supported in $\mathrm{Xd}-\mathrm{C}$, but not the goto statement. A continue statement shall appear only in the body of a loop. A break statement terminates execution of the smallest enclosing switch or iteration statement. A return statement appears only in the body of a function.

An Xd-C program is composed of a list of declarations, a list of functions 
and a main function. It can be defined as follows:

\begin{tabular}{|c|c|c|c|}
\hline Array & array & $::=$ & $\begin{array}{l}\tau i d[n]|\tau i d[m][n]| \tau i d[n]=\left\{(e,)^{*} e\right\} \\
\left|\tau i d[]=\left\{(e,)^{*} e\right\}\right| \tau i d[m][n]=\left\{(e,)^{*} e\right\} \\
\mid \tau i d[m][n]=\left\{\left(\left\{(e,)^{*} e\right\},\right)^{*}\left\{(e,)^{*} e\right\}\right\}\end{array}$ \\
\hline Structure & structure & $::=$ & struct $i d_{1}\left\{\left(\tau i d_{2} ;\right)^{+}\right\}$ \\
\hline Variable list & varlist & $::=$ & $i d|i d=e|$ varlist, varlist \\
\hline Declaration & $P d$ & $::=$ & $\tau$ varlist $\mid$ array $\mid$ structure \\
\hline Parameter & par & $::=$ & $\mid\left(\tau i d_{2},\right)^{*}\left(\tau i d_{2}\right)$ \\
\hline Function & funct & $::=$ & $\begin{array}{l}{[\tau \mid \text { void }] \text { id } d_{1}(\text { par })\left\{(P d ;)^{*} c s\right\}} \\
\mid \text { extern }[\tau \mid \text { void }] \text { id } d_{1}(\text { par })\end{array}$ \\
\hline Program & $P$ & $::=$ & $\begin{array}{l}(P d ;)^{*}(\text { funct } ;)^{*} \\
\text { int main }(\text { int argc, char } * * \text { argv })\left\{(P d ;)^{*} c\right.\end{array}$ \\
\hline
\end{tabular}

where $\tau i d[n]$ defines a one dimensional array $i d$ having $n$ elements of type $\tau$ while $\tau i d[m][n]$ defines a two dimensional array $i d$ having $m \times n$ elements of type $\tau ; i d=e$ defines an initialization of $i d$ except for structself; $[\tau \mid$ void $] i d_{1}((\tau$ $\left.\left.i d_{2},\right)^{*}\left(\tau i d_{2}\right)\right)\left\{(P d ;)^{*} c s\right\}$ defines a function $i d_{1}$ with each parameter $i d_{2}$ of type $\tau$ and a return value of type $\tau$ or void; $[\tau \mid$ void $] i d_{1}()\left\{(P d ;)^{*} c s\right\}$ defines a function $i d_{1}$ with no parameter.

Summary: As we can see, some constructs and facilities in ANSI-C (C89) are not supported in $\mathrm{Xd}-\mathrm{C}$. In the following, we show a key negative list which $\mathrm{Xd}-\mathrm{C}$ does not support.

(1) goto statement;

(2) union structure;

(3) $++e$ and $--e$ expressions;

(4) $(a=b, b=c, d=(f(x), 0))$ comma statements;

(5) op $=$ compound assignments where op ::=+|-|*|/|\%|>>|<<|\&| $\left.1\right|^{\wedge}$;

(6) struct $A$ a; $a=\{(\operatorname{void}() *) b,(\operatorname{void}() *) c\}$ structure assignments;

(7) $x=y=z$ continuous assignments;

(8) typedef, extern, static, auto and register storage-class specifiers;

(9) const and volatile type qualifiers;

(10) local variables in a block;

(11) nested cases in a switch statement;

(12) assignment expressions such as if $((y=f u n())==x)$;

(13) function pointers pointing to external functions;

(14) functions that accept a variable number of arguments.

In fact, the constructs and facilities in the above negative list except for goto statement can be implemented by $\mathrm{Xd}-\mathrm{C}$ although the implementation might be tedious. Therefore, Xd-C is a reasonable subset of ANSI-C (C89) in practice. 


\subsection{Operational semantics of $X d-C$}

The operational semantics of expressions and statements in $\mathrm{Xd}-\mathrm{C}$ is borrowed from [26] and given in Appendix A. The formal semantics of a large subset of $\mathrm{C}$ language called Clight is presented in [26. Clight features most of the types and operators of $\mathrm{C}$, including all arithmetic types, pointer, struct and union types, as well as all $\mathrm{C}$ control structures except goto statement, while $\mathrm{Xd}-\mathrm{C}$ supports all types, expressions and statements in Clight except union type.

The semantic elements including block references, memory locations, statement outcomes, evaluation environments, memory states, traces, program behaviors and operations over memory states and global environments are defined in Figure 1. Memory location $\ell$ is a pair of a memory block reference $b$ and a byte offset $\delta$ within this block. Statement outcome out indicates how an execution terminates: either normally by running to completion or prematurely via a jump statement. Global environment $G$ maps program-global variables and function names to memory block references, as well as those references corresponding to function pointers to the definitions of functions. Local environment $E$ maps function scoped variables to their memory block reference. Memory state $M$ maps memory block references to bounds and contents. Each memory block has lower and upper bounds $l o, h i$. B describes the program behavior. The basic operations over memory states (alloc, free, load and store) and global environments (functdef, symbol, globalnev and initmem) are also summarized. Note that, for functions returning "option" types, $\lfloor x\rfloor$ corresponds to success with return value $x$, and $\emptyset$ to failure.

The semantics is defined by the following judgements:

$$
\begin{array}{ll}
G, E \vdash l e, M \stackrel{l}{\Rightarrow} \ell & \text { (evaluation of left-value expressions) } \\
G, E \vdash e, M \Rightarrow v & \text { (evaluation of right-value expressions) } \\
G, E \vdash c s, M \stackrel{t}{\Rightarrow} \text { out }, M^{\prime} & \text { (evaluation of statements, terminating case) } \\
G \vdash \operatorname{funct}\left(v_{\text {args }}\right), M \stackrel{t}{\Rightarrow} v, M^{\prime} & \text { (evaluation of function invocations, terminating case) } \\
G, E \vdash \operatorname{cs}, M \stackrel{T}{\Rightarrow} \infty & \text { (evaluation of statements, diverging case) } \\
G \vdash \operatorname{funct}\left(v_{\text {args }}\right), M \stackrel{T}{\Rightarrow} \infty & \text { (evaluation of function invocations, diverging case) } \\
\vdash P \Rightarrow B & \text { (execution of whole programs) }
\end{array}
$$

Each judgement relates a syntactic element and an initial memory state $M$ to the result of executing this syntactic element. For a left-value expression le, the result is a location $\ell$ while for a right-value expression $e$, the result is its value $v$. For the execution of a statement $c s$ in the terminating case, the result is a statement outcome out and an updated memory state $M^{\prime}$ while in the diverging case, the result is $\infty$. For a function call funct $\left(v_{\text {args }}\right)$ in the terminating case, the result is its value $v$ and a memory state $M^{\prime}$ while in the diverging case, the result is $\infty$, where $v_{\text {args }}$ is a list of values of arguments. For the execution of a program $P$, it terminates with trace $t$ and exit code $n$ or diverges with trace $T$. 


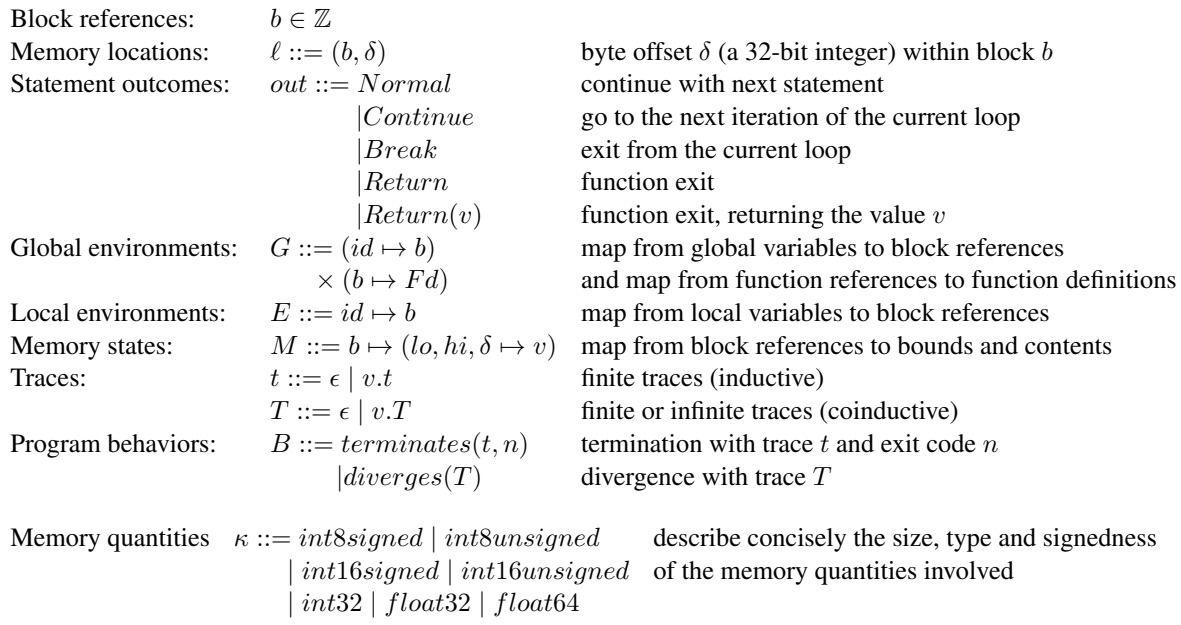

Operations over memory states:

$\operatorname{alloc}(M, l o, h i)=\left(M^{\prime}, b\right)$

Allocate a fresh block of bounds [lo,hi). Return extended memory $M^{\prime}$ and reference $b$ to fresh block.

Store value $v$ into one or several consecutive bytes (as determined by $\kappa$ ) at offset $n$ in block $b$ of

memory state $M$. If successful, return an updated memory state $M^{\prime}$.

Operations over global environments:

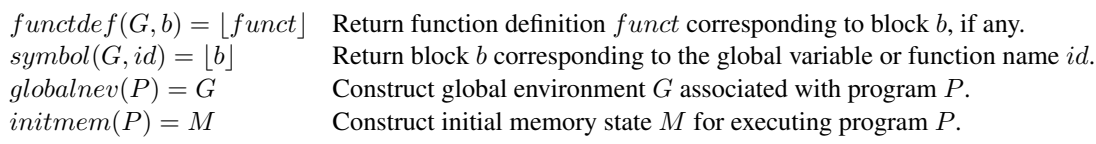

Figure 1: Semantic elements: block references, memory locations, statement outcomes, environments, memory states, etc

\section{MSVL}

MSVL is a Modeling, Simulation and Verification Language [20, 21, 22] which can be used to both model and execute a system. It is a subset of Projection Temporal logic (PTL). There are some statements in MSVL that cannot expressed by Xd-C, thus a suitable subset of MSVL can support Xd$\mathrm{C}$ well. This section briefly introduces the suitable subset of MSVL which is borrowed from [20, 22, 27, 28, 30, 31. 


\subsection{Syntax}

The left-value expression la, right-value expression $r a$ and Boolean expression $b$ in the subset of MSVL are inductively defined as follows:

$$
\begin{aligned}
& v::=i d|i d[r a]| i d\left[r a_{1}\right]\left[r a_{2}\right] \mid \text { la.v } \mid p t \rightarrow v \\
& h::=v \quad g::=v \\
& p t::=l a|\& l a|(\tau) r a\left|r a_{1}+r a_{2}\right| r a_{1}-r a_{1}\left|\operatorname{ext} g\left(r a_{1}, \ldots, r a_{k}\right)\right| \\
& \text { ext } h\left(r a_{1}, \ldots, r a_{k}, R V a l\right) \\
& l a::=v \mid * p t \\
& r a::=c|l a| \& l a|(\tau) r a| m_{1} p_{1} r a \mid r a_{1} \text { mop }_{2} r a_{2} \mid \text { if(b)then } r a_{1} \text { else } r a_{2}|\Theta r a| \\
& \text { ext } g\left(r a_{1}, \ldots, r a_{k}\right) \mid \operatorname{ext} h\left(r a_{1}, \ldots, r a_{k}, R V a l\right) \\
& \operatorname{mop}_{1}::=+|-|^{\sim} \\
& \operatorname{mop}_{2}::=+|-| *|/| \%|<<|>>\left.|\&| 1\right|^{\wedge} \\
& b::=\text { true } \mid \text { false } \mid r a_{1} \text { mrop } r a_{2}|\neg b| b_{1} \wedge b_{2} \mid b_{1} \vee b_{2} \\
& \text { mrop }::=<|>|<=|>=|=\mid !=
\end{aligned}
$$

\begin{tabular}{|c|c|c|}
\hline$m s::=$ & $\begin{array}{l}\text { empty } \\
\mid \text { skip } \\
\mid l a \Leftarrow r a \\
\mid l a:=r a \\
\mid m s_{1} \text { and } m s_{2} \\
\mid \text { next } m s \\
\mid m s_{1} ; m s_{2} \\
\mid \text { if }(b) \operatorname{then}\left\{m s_{1}\right\} \operatorname{else}\left\{m s_{2}\right\} \\
\mid \text { while }(b)\{m s\} \\
\mid h\left(r a_{1}, \ldots, r a_{k}\right) \\
\mid \operatorname{ext} h\left(r a_{1}, \ldots, r a_{k}\right)\end{array}$ & $\begin{array}{l}\text { Termination } \\
\text { Skip } \\
\text { Assignment } \\
\text { Unit Assignment } \\
\text { Conjunction } \\
\text { Next } \\
\text { Sequence } \\
\text { Conditional } \\
\text { While } \\
\text { Function call } \\
\text { External function call }\end{array}$ \\
\hline
\end{tabular}

where $v$ denotes a variable and $p t$ an expression of a pointer type. The explanations of $c, l a, \& l a$ and $(\tau) r a$ are the same as explanations of $c, l e, \& l e$ and $(\tau) e$ in Xd-C, respectively. $g\left(r a_{1}, \ldots, r a_{k}\right)$ is a function call of an external function while ext $h\left(r a_{1}, \ldots, r a_{k}, R V a l\right)$ is an external function call of an MSVL userdefined function, where $R V a l$ is the return value. An external call of function ext $h\left(r a_{1}, \ldots, r a_{k}, R V a l\right)$ means that we concern only the return value of the function but not the interval over which the function is executed. $\odot r a$ stands for the value of $r a$ at the previous state. We assume that all variables used are framed. The following are the elementary statements in the subset of MSVL:

The termination statement "empty" means that the current state is the final state of an interval. "skip" specifies one unit of time over an interval. The assignment " $l a \Leftarrow r a$ " indicates that $l a$ is assigned the value of $r a$ at the current state while "la $:=r a$ " means that the value of $l a$ at the next state equals the current value of $r a$ and the length of the interval is one unit of time. The conjunction statement " $m s_{1}$ and $m s_{2}$ " indicates that $m s_{1}$ and $m s_{2}$ are executed concurrently. "next $m s$ " means that $m s$ will be executed at the next state. 
" $m s_{1} ; m s_{2}$ " means that $m s_{1}$ is executed until its termination from this time point then $m s_{2}$ is executed or $m s_{1}$ is infinitely executed. " $h\left(r a_{1}, \ldots, r a_{k}\right)$ " and "ext $h\left(r a_{1}, \ldots, r a_{k}\right)$ " are internal and external function calls, respectively. The meanings of other statements are the same as Xd-C. Note that all the above statements are defined by PTL formulas in Appendix B.

In addition, data type $\tau$ in MSVL [28] is defined the same as in Xd-C. An MSVL program $P$ can be defined as follows:

\begin{tabular}{|c|c|c|c|}
\hline Array & marray & $::=$ & $\begin{array}{l}\tau i d[n]|\tau i d[m][n]| \tau i d[n] \Leftarrow\left\{(r a,)^{*} r a\right\} \\
\mid \tau i d[m][n] \Leftarrow\left\{(r a,)^{*} r a\right\} \\
\mid \tau i d[m][n] \Leftarrow\left\{\left(\left\{(r a,)^{*} r a\right\},\right)^{*}\left\{(r a,)^{*} r a\right\}\right\}\end{array}$ \\
\hline Structure & mstructure & $::=$ & struct $i d_{1}\left\{\left(\tau i d_{2} \text { and }\right)^{*} \tau i d_{2}\right\}$ \\
\hline Variable list & mvarlist & $::=$ & $i d|i d \Leftarrow r a|$ varlist, varlist \\
\hline Declaration & $m P d$ & $::=$ & $\tau$ mvarlist $\mid$ marray $\mid$ mstructure \\
\hline Function body & $\phi$ & $::=$ & $(m P d ;)^{*} m s$ \\
\hline Function & mfunct & $::=$ & $\begin{array}{l}\text { function } i d_{1}\left(\left(\tau i d_{2},\right)^{*} \tau R V a l\right)\{\phi\} \\
\mid \text { function } i d_{1}\left(\left(\tau i d_{2},\right)^{*} \tau i d_{2}\right)\{\phi\} \\
\text { | function } i d_{1}()\{\phi\}\end{array}$ \\
\hline Program & $P$ & $::=$ & $(m P d ;)^{*}(m f u n c t ;)^{*} m s$ \\
\hline
\end{tabular}

where in a structure definition struct $i d_{1}\left\{\left(\tau i d_{2} \text { and }\right)^{*} \tau i d_{2}\right\}$, and is used to connect each member $i d_{2}$ of struct $i d_{1}$; in a function fragment function $i d_{1}\left(\left(\tau i d_{2},\right)^{*} \tau\right.$ $R V a l)\{\phi\}, R V a l$ is the return value; function $i d_{1}\left(\left(\tau i d_{2},\right)^{*} \tau i d_{2}\right)\{\phi\}$ and function $i d_{1}()\{\phi\}$ define functions without a return value.

\subsection{Operational semantics}

The operational semantics of expressions and statements in the subset of MSVL is borrowed from [22, 27, 28]

\subsubsection{Notation}

In order to directly express programs $p_{1}, \ldots, p_{k}$ connected by $\wedge$ and $\vee$, the following notations are defined.

$$
(1) \wedge\left\{p_{1}, \ldots, p_{k}\right\} \stackrel{\text { def }}{=} p_{1} \wedge \ldots \wedge p_{k}(k \geq 1) \quad(2) \vee\left\{p_{1}, \ldots, p_{k}\right\} \stackrel{\text { def }}{=} p_{1} \vee \ldots \vee p_{k}(k \geq 1)
$$

Let $\mathcal{V}$ denote a set of variables and $D$ the set of all data in type $\tau$ needed by us. A state $s$ is defined as a pair $\left(s^{l}, s^{r}\right)$, where $s^{l}$ is a mapping $\mathcal{V} \longrightarrow \mathbb{Z} \times N_{0}$ and $s^{r}$ a mapping $\mathcal{V} \longrightarrow D$. $\operatorname{Dom}(s)$ is defined as $\operatorname{Dom}(s)=\operatorname{Dom}\left(s^{l}\right)=\operatorname{Dom}\left(s^{r}\right)=\mathcal{V}$. The $i$ th state $s_{i}=\left(s_{i}^{l}, s_{i}^{r}\right) . s_{i}^{l}(x)$ denotes the location of variable $x$ in type $\tau$ at state $s_{i}$ and $s_{i}^{r}(x)$ the value of $x$ in type $\tau$ at $s_{i}$. Note that for an array $a[n]$ in type $\tau$, if $s_{i}^{l}(a)=(b, 0), s_{i}^{l}(a[j])=(b, j *$ sizeof $(\tau))$ for $0 \leq j<n$ and for an array $a[m][n]$ in type $\tau$, if $s_{i}^{l}(a)=(b, 0), s_{i}^{l}(a[j][k])=(b,(j * n+k) * \operatorname{sizeof}(\tau))$ for $0 \leq j<m$ and $0 \leq k<n$. Let $\sigma=\left\langle s_{0}, \ldots\right\rangle$ denote an interval. Over an interval $\sigma$, if a variable $x$ is not released, $s_{i}^{l}(x)=s_{i+1}^{l}(x)$, for $i \geq 0$; otherwise, $x$ is removed from the state.

We have two types of configurations, one for expressions, and the other for programs. A configuration of a program $p$ is a quadruple $\left(p, \sigma_{i-1}, s_{i}, i\right)$, where $\sigma_{i-1}=\left\langle s_{0}, \ldots, s_{i-1}\right\rangle(i>0)$ records information of all states, $s_{i}$ is the current 
state and $i$ counts the number of states in $\sigma_{i-1}$. Further, let the initial configuration be $c_{0}=\left(p, \epsilon, s_{0}, 0\right)$. When a program $p$ is terminating, it is reduced to true and the state is written as $\emptyset$. So the final configuration is $c_{f}=(\operatorname{true}, \sigma, \emptyset,|\sigma|+1)$ if it can terminate, otherwise, there will always be a transition from the current state to the next one. Let $\hookrightarrow$ denote the congruence relation between configurations. $c \stackrel{*}{\longmapsto} c^{\prime}$ implies that $c$ is transformed to $c^{\prime}$ by zero or several steps within a state. Notation $\rightarrow$ is a binary relation between two configurations with different states. $c \stackrel{*}{\rightarrow} c^{\prime}$ implies that $c$ is transformed to $c^{\prime}$ after zero or several states and $c \stackrel{+}{\rightarrow} c^{\prime}$ implies that $c$ is transformed to $c^{\prime}$ after at least one state.

Similarly, for an arithmetic (or Boolean) expression $a$, the configuration is $\left(a, \sigma_{i-1}, s_{i}, i\right)$. The evaluation of a left-value is of the form $\left(l a, \sigma_{i-1}, s_{i}, i\right) \stackrel{l}{\Rightarrow}$ $(b, \delta)$, which means that the location of $l a$ is $(b, \delta)$ at state $s_{i}$. The evaluation of a right-value is of the form $\left(r a, \sigma_{i-1}, s_{i}, i\right) \Downarrow n$, which means that the value of $r a$ is $n$ at state $s_{i}$.

For accessing the locations and values of variables at a state, the following notations are used. Let $n$ be a value in $D, x$ and $y$ variables. $\left(s_{i}^{l}, s_{i}^{r}[n / x]\right)$ means that the location of $x$ is not changed and the value of $x$ is changed to $n$, and other variables are not changed at $s_{i}$. Thus, we have,

$$
\left(s_{i}^{l}, s_{i}^{r}[n / x]\right)(y)= \begin{cases}\left(s_{i}^{l}(y), n\right) & y=x \\ \left(s_{i}^{l}(y), s_{i}^{r}(y)\right) & y \neq x\end{cases}
$$

\subsubsection{Evaluation of expressions}

Rules in Tables 1 and 2 are evaluation rules of arithmetic expressions for leftvalues and right-values, respectively. $\operatorname{sizeof}(\tau)$ returns the storage size of type $\tau$ and type $(a)$ the type of expression $a$. field_offset $(v, \varphi)$ returns the byte offset of the field named $v$ in a struct whose field list is $\varphi$ or $\emptyset$ if $v$ does not appear in $\varphi$. $p \operatorname{tr}(b, \delta)$ denotes a pointer value pointing to $(b, \delta)$. Rule L1 deals with variables, L2 and L3 elements of arrays, L4 and L5 members of structure variables and L6 pointer dereferencing. Rule R1 tackles with constants. R2 deals with expressions which can also appear in left-value position. R3 handles expressions with the address-of operator, R4 type cast operators, R5 - R8 arithmetic operators and R9 the previous $(\odot)$ operator.

Rules B1 - B6 in Table 3 tackle with Boolean expressions.

\subsubsection{State reduction}

The semantic equivalence rules regarding programs are formalized in Table 4. Rule SKIP is concerned with statement skip and $\bigcirc$ empty specifies one unit of time over an interval. UASS handles unit assignment statement $l a:=r a$. la is assigned by $r a$ at the next state and it takes one unit of time. The conjunction statement $m s_{1}$ and $m s_{2}$ can be expressed by $\wedge\left\{m s_{1}, m s_{2}\right\}$. We define more as more $\stackrel{\text { def }}{=} \bigcirc$ true. $\bigcirc m s$ implies that $m s$ will be executed at the next state and more means that the current interval is not yet over. $\square m s$ is handled by rule ALW depending on more or empty encountered in programs. In order to keep consistence of operational semantics with the Xd-C sequential statement 
Table 1: Evaluation rules of arithmetic expressions for left-values

\begin{tabular}{|c|c|}
\hline L1 & $\left(i d, \sigma_{i-1}, s_{i}, i\right) \stackrel{l}{\Rightarrow} s_{i}^{l}(i d)$ \\
\hline I? & $\left(r a, \sigma_{i-1}, s_{i}, i\right) \Downarrow n \quad\left(i d, \sigma_{i-1}, s_{i}, i\right) \stackrel{l}{\Rightarrow}(b, 0)$ \\
\hline & $\left(i d[r a], \sigma_{i-1}, s_{i}, i\right) \stackrel{l}{\Rightarrow}(b, n * \operatorname{sizeof}(\tau))$ \\
\hline I 3 & $\left(r a_{1}, \sigma_{i-1}, s_{i}, i\right) \Downarrow n_{1} \quad\left(r a_{2}, \sigma_{i-1}, s_{i}, i\right) \Downarrow n_{2} \quad\left(i d, \sigma_{i-1}, s_{i}, i\right) \stackrel{l}{\Rightarrow}(b, 0)$ \\
\hline I4 & $\begin{array}{l}\qquad\left(i d\left[r a_{1}\right]\left[r a_{2}\right], \sigma_{i-1}, s_{i}, i\right) \stackrel{l}{\Rightarrow}\left(b,\left(n_{1} * n+n_{2}\right) * \operatorname{sizeof}(\tau)\right) \\
\text { where } n \text { is the number of elements in each row of } i d\left[r a_{1}\right]\left[r a_{2}\right] \text { and } \\
\tau \text { is the type of } i d\left[r a_{1}\right]\left[r a_{2}\right] \\
\qquad \begin{aligned}\left(l a, \sigma_{i-1}, s_{i}, i\right) \stackrel{l}{\Rightarrow}(b, \delta) \quad \text { type }(l a)=\text { struct } i d^{\prime}\{\varphi\} \\
\text { field_offset }(v, \varphi)=\left\lfloor\delta^{\prime}\right\rfloor\end{aligned}\end{array}$ \\
\hline L4 & $\left(\right.$ la.v, $\left.\sigma_{i-1}, s_{i}, i\right) \stackrel{l}{\Rightarrow}\left(b, \delta+\delta^{\prime}\right)$ \\
\hline I 5 & $\begin{array}{c}\left(p t, \sigma_{i-1}, s_{i}, i\right) \Downarrow p \operatorname{ptr}(b, \delta) \quad \text { type }(p t)=\text { struct } i d^{\prime}\{\varphi\} * \\
\text { field_offset }(v, \varphi)=\left\lfloor\delta^{\prime}\right\rfloor\end{array}$ \\
\hline LU & $\begin{aligned} &\left(p t \rightarrow v, \sigma_{i-1}, s_{i}, i\right) \stackrel{l}{\Rightarrow}\left(b, \delta+\delta^{\prime}\right) \\
& \underline{\left(p t, \sigma_{i-1}, s_{i}, i\right) \Downarrow p \operatorname{tr}(b, \delta)}\end{aligned}$ \\
\hline & $\overline{\left(* p t, \sigma_{i-1}, s_{i}, i\right) \stackrel{l}{\Rightarrow}(b, \delta)}$ \\
\hline
\end{tabular}

Table 2: Evaluation rules of arithmetic expressions for right-values

\begin{tabular}{|c|c|}
\hline $\mathrm{R} 1$ & $\left(c, \sigma_{i-1}, s_{i}, i\right) \Downarrow c$ \\
\hline B2 & $\left(l a, \sigma_{i-1}, s_{i}, i\right) \stackrel{l}{\Rightarrow} s_{i}^{l}(x)$ \\
\hline $\mathrm{n} 2$ & $\overline{\left(l a, \sigma_{i-1}, s_{i}, i\right) \Downarrow s_{i}^{r}(x)}$ \\
\hline B3 & $\left(l a, \sigma_{i-1}, s_{i}, i\right) \stackrel{l}{\Rightarrow}(b, \delta)$ \\
\hline & $\begin{aligned} &\left(\& l a, \sigma_{i-1}, s_{i}, i\right) \Downarrow p \operatorname{tr}(b, \delta) \\
&\left(r a, \sigma_{i-1}, s_{i}, i\right) \Downarrow n_{1}\end{aligned}$ \\
\hline $\mathrm{R} 4$ & 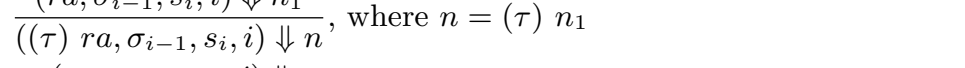 \\
\hline R5 & $\frac{\left(r a_{1}, \sigma_{i-1}, s_{i}, i\right) \Downarrow n_{1}}{\left(m o p_{1} r a_{1}, \sigma_{i-1}, s_{i}, i\right) \Downarrow n}$, where $n=\operatorname{mop}_{1} n_{1}$ \\
\hline R6 & $\frac{\left(r a_{1}, \sigma_{i-1}, s_{i}, i\right) \Downarrow n_{1} \quad\left(r a_{2}, \sigma_{i-1}, s_{i}, i\right) \Downarrow n_{2}}{\left(r a_{1} m o p_{2} r a_{2}, \sigma_{i-1}, s_{i}, i\right) \Downarrow n}$, where $n=n_{1} \operatorname{mop}_{2} n_{2}$ \\
\hline R7 & $\frac{\left(b, \sigma_{i-1}, s_{i}, i\right) \Downarrow \text { true } \quad\left(r a_{1}, \sigma_{i-1}, s_{i}, i\right) \Downarrow n_{1}}{\left(i f(b) \text { then } r a_{1} \text { else } r a_{2}, \sigma_{i-1}, s_{i}, i\right) \Downarrow n_{1}}$ \\
\hline $\mathrm{R} 8$ & $\frac{\left(b, \sigma_{i-1}, s_{i}, i\right) \Downarrow \text { false } \quad\left(r a_{2}, \sigma_{i-1}, s_{i}, i\right) \Downarrow n_{2}}{\left(\text { if }(b) \text { then } r a_{1} \text { else } r a_{2}, \sigma_{i-1}, s_{i}, i\right) \Downarrow n_{2}}$ \\
\hline R9 & $\frac{\left(r a, \sigma_{i-m-1}, s_{i-m}, i-m\right) \Downarrow n}{\left(\bigodot^{m} r a, \sigma_{i-1}, s_{i}, i\right) \Downarrow n}$, where $m \leq i$ \\
\hline
\end{tabular}

$r ; m s_{2}$ in which $r$ could execute over an infinite interval, in this paper, we use weak chop ';' instead of strong chop '; ' as most of time in MSVL programs. As a matter of fact, the two operators can be defined by each other. Formally, $r ; m s_{2} \stackrel{\text { def }}{=}\left(r ; s_{s} m s_{2}\right) \vee(r \wedge \square$ more $)$ and $r ; s_{s} \stackrel{\text { def }}{=}\left(r \wedge \diamond \varepsilon ; m s_{2}\right)$. Thus, rule CHOP deals with $\left(r ; m s_{2}\right)$ in light of the structure of program $r$ in four forms. In the case of $\left(r \equiv \wedge\left\{w, m s_{1}\right\}\right)$, and $w$ being a state program or true, $\left(r ; m s_{2}\right)$ is 
Table 3: Evaluation rules of Boolean expressions

\begin{tabular}{|c|c|c|}
\hline B1 & $\left(\right.$ true $\left., \sigma_{i-1}, s_{i}, i\right) \Downarrow$ true & \\
\hline B2 & (false, $\left.\sigma_{i-1}, s_{i}, i\right) \Downarrow$ false & \\
\hline B3 & $\frac{\left(r a_{1}, \sigma_{i-1}, s_{i}, i\right) \Downarrow n_{1} \quad\left(r a_{2}, \sigma_{i-1}, s_{i}, i\right) \Downarrow n_{2}}{\left(r a_{1} \text { mrop } r a_{2}, \sigma_{i-1}, s_{i}, i\right) \Downarrow t} t$ & $= \begin{cases}\text { true } & \text { if } n_{1} \text { mrop } n_{2} \\
\text { false } & \text { otherwise }\end{cases}$ \\
\hline B4 & $\frac{\left(b, \sigma_{i-1}, s_{i}, i\right) \Downarrow \text { true }}{\left(\neg b, \sigma_{i-1}, s_{i}, i\right) \Downarrow \text { false }} \quad \frac{\left(b, \sigma_{i-1}, s_{i}, i\right) \Downarrow \text { false }}{\left(\neg b, \sigma_{i-1}, s_{i}, i\right) \Downarrow \text { true }}$ & \\
\hline B5 & $\frac{\left(b_{1}, \sigma_{i-1}, s_{i}, i\right) \Downarrow t_{1} \quad\left(b_{2}, \sigma_{i-1}, s_{i}, i\right) \Downarrow t_{2}}{\left(b_{1} \wedge b_{2}, \sigma_{i-1}, s_{i}, i\right) \Downarrow t}, t=\{$ & $\begin{array}{l}\text { if } b_{1}=\text { true } \\
\text { and } b_{2}=\text { true } \\
\text { otherwise }\end{array}$ \\
\hline B6 & $\frac{\left(b_{1}, \sigma_{i-1}, s_{i}, i\right) \Downarrow t_{1} \quad\left(b_{2}, \sigma_{i-1}, s_{i}, i\right) \Downarrow t_{2}}{\left(b_{1} \vee b_{2}, \sigma_{i-1}, s_{i}, i\right) \Downarrow t}, t=\{$ & $\begin{array}{l}\text { if } b_{1}=\text { true } \\
\text { or } b_{2}=\text { true } \\
\text { otherwise }\end{array}$ \\
\hline
\end{tabular}

reduced to $\left(\wedge\left\{w, m s_{1} ; m s_{2}\right\}\right)$; in the case of $\left(r \equiv \bigcirc m s_{1}\right),\left(r ; m s_{2}\right)$ is transformed to $\left(\bigcirc\left(m s_{1} ; m s_{2}\right)\right)$; in the case of $(r \equiv$ empty $),\left(r ; m s_{2}\right)$ is reduced to $m s_{2}$; and in case of ( $r \equiv \square$ more $),\left(r ; m s_{2}\right)$ is reduced to $\square$ more. Rule IF transforms the conditional statement to its equivalent program according to the definition. Rule WHL transforms the while statement to an equivalent conditional statement.

Table 4: Semantic equivalence rules of framed programs

\begin{tabular}{|c|l|}
\hline SKIP & skip $\equiv \bigcirc$ empty \\
UASS & If $\left(l a, \sigma_{i-1}, s_{i}, i\right) \stackrel{l}{\Rightarrow} s_{i}^{l}(x)$ and $\left(r a, \sigma_{i-1}, s_{i}, i\right) \Downarrow n$ then \\
& $l a:=r a \equiv \bigcirc(x \Leftarrow n \wedge$ empty $)$ \\
AND & $m s_{1}$ and $m s_{2} \equiv \wedge\left\{m s_{1}, m s_{2}\right\}$ \\
NEXT & next $m s \equiv \wedge\{\bigcirc m s, \square$ more $\}$ \\
ALW & $(1) \wedge\{\square m s$, empty $\} \equiv \wedge\{m s$, empty $\}$ \\
& $(2) \wedge\{\square m s$, more $\} \equiv \wedge\{m s, \bigcirc \square m s\}$ \\
CHOP & $(1) \wedge\left\{w, m s_{1}\right\} ; m s_{2} \equiv \wedge\left\{w, m s_{1} ; m s_{2}\right\}$ \\
& $(2) \bigcirc m s_{1} ; m s_{2} \equiv \bigcirc\left(m s_{1} ; m s_{2}\right)$ \\
& $(3)$ empty; $m s_{2} \equiv m s_{2}$ \\
IF & if $(b)$ then $\left\{m s_{1}\right\}$ else $\left\{m s_{2}\right\} \equiv\left(b \wedge m s_{1}\right) \vee\left(\neg b \wedge m s_{2}\right)$ \\
WHL & while $(b)\{m s\} \equiv \operatorname{if}(b) \operatorname{then}\{m s \wedge \operatorname{more} ;$ while $(b)\{m s\}\}$ else $\{$ empty $\}$ \\
\hline
\end{tabular}

Semantic equivalence rules regarding true and false are listed in Table 5 . We use $p$ to represent an MSVL program.

Table 5: Semantic equivalence rules of truth values

\begin{tabular}{|l|l|l|l|l|l|}
\hline $\mathrm{F} 1$ & $\wedge\{$ false,$p\} \equiv$ false & $\mathrm{F} 2$ & $\vee\{p$, false $\} \equiv p$ & $\mathrm{~F} 3$ & $\wedge\{p, \neg p\} \equiv$ false \\
$\mathrm{T} 1$ & $\wedge\{p$, true $\} \equiv p$ & $\mathrm{~T} 2$ & $\vee\{p$, true $\} \equiv \operatorname{true}$ & $\mathrm{T} 3$ & $\vee\{p, \neg p\} \equiv$ true \\
\hline
\end{tabular}

The following rules are concerned with assignments.

MIN1 If $\exists j, 1 \leq j \leq n,\left(l a_{j}, \sigma_{i-1}, s_{i}, i\right) \stackrel{l}{\Rightarrow} s_{i}^{l}\left(x_{j}\right)$ and $\left(r a_{j}, \sigma_{i-1}, s_{i}, i\right) \Downarrow n_{j}$, then $\left(\wedge\left\{p, \wedge_{k=1}^{n}\left\{l a_{k} \Leftarrow r a_{k}\right\}\right\}, \sigma_{i-1}, s_{i}, i\right) \longmapsto$ 
$\left(\wedge\left\{p, \wedge_{k=1, k \neq j}^{n}\left\{l a_{k}\left[n_{j} / x_{j}\right] \Leftarrow r a_{k}\left[n_{j} / x_{j}\right]\right\}\right\}, \sigma_{i-1},\left(s_{i}^{l}, s_{i}^{r}\left[n_{j} / x_{j}\right]\right), i\right)$.

If $l a_{j}$ can be evaluated to the location of a variable $x_{j}$ and $r a_{j}$ a constant $n_{j}$, then conjunct $l a_{j} \Leftarrow r a_{j}$ is eliminated from the program in the configuration, where $x_{j}$ is set to $n_{j}$ at state $s_{i}$. $l a_{k}\left[n_{j} / x_{j}\right]\left(r a_{k}\left[n_{j} / x_{j}\right]\right)$ means that variable $x_{j}$ is replaced by value $n_{j}$ in $l a_{k}\left(r a_{k}\right)$ for $1 \leq k \leq n$ and $k \neq j$.

MIN2 If $\left(\bigodot x, \sigma_{i-1}, s_{i}, i\right) \Downarrow n(i \geq 1)$ and there is no state component $l a \Leftarrow r a$ in $p$, where $\left(l a, \sigma_{i-1}, s_{i}, i\right) \stackrel{l}{\Rightarrow} s_{i}^{l}(x)$, then $\left(p, \sigma_{i-1}, s_{i}, i\right) \longmapsto\left(p, \sigma_{i-1},\left(s_{i}^{l}, s_{i}^{r}[n / x]\right), i\right)$.

If there is no assignment to variable $x$ at the current state, $x$ keeps its previous value.

Actually, once all of the variables involved in the current state have been set, the remained subprogram is of the forms, $\bigcirc \mathrm{ms}$ or empty. Rule TR1 in Table 6 deals with the former and rule TR2 the latter. Concretely, the transition of $\left(\bigcirc m s, \sigma_{i-1}, s_{i}, i\right)$ means that $m s$ will be executed at next state $s_{i+1}$, and current state $s_{i}$ needs to be appended to $\sigma_{i-1}$. So $i$, the number of states in $\sigma_{i-1}$, need plus one. The transition of (empty, $\left.\sigma_{i-1}, s_{i}, i\right)$ means that $s_{i}$ is appended to $\sigma_{i-1}$ and the final configuration $\left(\right.$ true $\left., \sigma_{i-1} \cdot\left\langle s_{i}\right\rangle, \emptyset, i+1\right)$ is reached.

Table 6: Interval transition rules

\begin{tabular}{|l|l|}
\hline TR1 & $\left(\bigcirc m s, \sigma_{i-1}, s_{i}, i\right) \rightarrow\left(m s, \sigma_{i}, s_{i+1}, i+1\right)$ \\
TR2 & $\left(\right.$ empty $\left., \sigma_{i-1}, s_{i}, i\right) \rightarrow\left(\right.$ true $\left., \sigma_{i}, \emptyset, i+1\right)$ \\
\hline
\end{tabular}

\subsubsection{Type declaration statement}

The set of basic data types $\mathcal{T}_{b}$ is defined as follows:

$$
\begin{aligned}
\mathcal{T}_{b} \stackrel{\text { def }}{=} & \left\{\text { int, float, } \operatorname{char}, \operatorname{int}\langle\rangle, \text { float }\langle\rangle, \operatorname{char}\langle\rangle, \operatorname{int}\left[n_{0}\right], \operatorname{int}\left[n_{0}\right]\left[n_{1}\right],\right. \\
& \text { float } \left.\left[n_{0}\right], \text { float }\left[n_{0}\right]\left[n_{1}\right], \operatorname{char}\left[n_{0}\right], \operatorname{char}\left[n_{0}\right]\left[n_{1}\right]\right\} .
\end{aligned}
$$

where $n_{0}, n_{1} \in N_{0}$. Note that when an array is declared, the number of elements of the array needs to be specified. For each basic data type $T \in \mathcal{T}_{b}$, a point type $T *$ is introduced and the set of the pointer types are $\mathcal{T}_{p b}=\left\{T * \mid T \in \mathcal{T}_{b}\right\}$. Let $\mathcal{T}_{s}$ denote a countable set of all possible names of struct types. For each struct type $S \in \mathcal{T}_{s}, S *$ denotes the pointer type to $S$ and the set of pointer types to struct types is $\mathcal{T}_{p s}=\left\{S * \mid S \in \mathcal{T}_{s}\right\}$. Let $\mathcal{T}^{d} \stackrel{\text { def }}{=} \mathcal{T}_{b} \cup \mathcal{T}_{p b} \cup \mathcal{T}_{s} \cup \mathcal{T}_{p s}$ be the union of basic data types, struct types and their corresponding pointer types. We define predicates $I S_{T}(\cdot)$, which means "is of type $T$ ", for each type $T \in \mathcal{T}^{d}$.

$$
I S_{T}: T \longrightarrow B \text { for } T \in \mathcal{T}^{d}
$$

For every type $T \in \mathcal{T}^{d}$, the formula $I S_{T}(v)$ represents $v$ is a variable of type $T$.

Using these predicates, we define the type declaration statements as a derived PTL formula.

$$
T x \stackrel{\text { def }}{=} \square I S_{T}(x), \quad \text { for } T \in \mathcal{T}_{b} \cup \mathcal{T}_{p b}
$$


Suppose a struct $S$ is defined with types of its members being $S_{1} *, \ldots, S_{n} * \in$ $\mathcal{T}_{p s}(n \geq 0)$. Such a struct definition $S$ is called legal if each struct $S_{i}(1 \leq$ $i \leq n)$ is defined no later than the definition of $S$ and illegal otherwise. We call a program with illegal struct definitions unhealthy. To deal with unhealthy programs, a special proposition $\mu$ meaning "unhealthy" is introduced. Moreover, in order to make sure that the values of a struct type should be consistent with the struct definition in terms of members and their types, for each struct $S$, a system variable mem $_{S}$ is used. We use notation $F P F\left(E_{1}, E_{2}\right)$ to denote the set of all finite partial functions from a set $E_{1}$ to another set $E_{2}$, i.e., all finite subsets of $E_{1} \times E_{2}$ that are partial functions. Formally,

$F P F\left(E_{1}, E_{2}\right) \stackrel{\text { def }}{=}\left\{E \mid E \subset E_{1} \times E_{2}, E\right.$ is finite and $(u, v),\left(u, v^{\prime}\right) \in E$ implies $\left.v=v^{\prime}\right\}$.

Variable mem $_{S}$ takes a special type $M E M T Y P E \stackrel{\text { def }}{=} F P F\left(\mathcal{V}, \mathcal{T}^{d} \backslash \mathcal{T}_{s}\right)$. The interpretation of mem $_{S}$ is of the form $\left\{\left(a_{1}, T_{1}\right), \ldots,\left(a_{k}, T_{k}\right)\right\}(k \geq 1)$. We define the struct definition as follows:

$$
\begin{aligned}
& \text { struct } S\left\{T_{1} a_{1} \text { and } \ldots \text { and } T_{k} a_{k}\right\} \stackrel{\text { def }}{=} \\
& \square\left(r_{S} \wedge \operatorname{mem}_{S}=\left\{\left(a_{1}, T_{1}\right), \ldots,\left(a_{k}, T_{k}\right)\right\}\right) \wedge\left(r_{S_{1}} \wedge \ldots \wedge r_{S_{n}} \vee \mu\right)
\end{aligned}
$$

where $\left\{S_{1} *, \ldots, S_{n} *\right\}=\left\{T_{1}, \ldots, T_{k}\right\} \cap \mathcal{T}_{p s}$ is the set of struct pointer types in $T_{1}, \ldots, T_{k}$ and $r_{S}$ (resp. $r_{S_{i}}$ ) denotes whether $S$ (resp. $S_{i}$ ) is defined or not.

We call a variable $x$ is consistent with struct $S$ in terms of members, if it has exactly the members and their types defined by $S$. To check the consistency of $x$ with $S$ in terms of members, we define a predicate $C o n$ as follows:

$$
\text { Con : } \begin{aligned}
& \bigcup_{S \in \mathcal{T}_{s}}(S \times M E M T Y P E \longrightarrow B) \\
& (v, S),\left\{\left(a_{1}, T_{1}\right), \ldots,\left(a_{k}, T_{k}\right)\right\} \mapsto \text { true, if } v \text { is of the form } \\
& \left\{\left(a_{1},\left(v_{1}, T_{1}\right)\right), \ldots,\left(a_{k},\left(v_{k}, T_{k}\right)\right)\right\} ; \\
& (v, S),\left\{\left(a_{1}, T_{1}\right), \ldots,\left(a_{k}, T_{k}\right)\right\} \mapsto \text { false, otherwise. }
\end{aligned}
$$

$\operatorname{Con}\left(x\right.$, mem $\left._{S}\right)$ indicates $x$ is consistent with $S$ in terms of members. To define variable declarations, we first extend the definition of unhealthy programs. A variable declaration $S x$ or $S * x$ is called legal if a struct definition struct $S\{\ldots\}$ is no later than the variable declaration and illegal otherwise. A program with illegal struct definitions or variable declarations is called unhealthy. Proposition $\mu$ is still used to mean "unhealthy". Then the struct variable and struct pointer variable declarations can be specified as follows:

$$
\begin{aligned}
& S x \stackrel{\text { def }}{=} r_{S} \wedge \square\left(I S_{S}(x) \wedge \operatorname{Con}\left(x, \text { mem }_{S}\right)\right) \vee \neg r_{S} \wedge \mu, \\
& S * x \stackrel{\text { def }}{=} \quad r_{S} \wedge \square I S_{S *}(x) \vee \neg r_{S} \wedge \mu .
\end{aligned}
$$

\subsubsection{Function}

Two kinds of functions can be used in MSVL: external functions, written in other programming languages such as $\mathrm{C}$, and user-defined functions written in MSVL. There are also two kinds of function calls: external call and internal call. For an external call, the interval over which the callee function is executed 
is ignored, while for an internal call, the interval over which the callee function is executed is inserted and concatenated with the main interval over which the caller function is executed. External functions can only be invoked as an external call while user-defined MSVL functions can be invoked as either an external call or internal call. As in $\mathrm{C}$ language, function calls can appear in expressions and statements in MSVL programs. The evaluation rules of function calls in expressions and semantic equivalence rules of function calls in statements are given in the following parts.

Evaluation rules of function calls in expressions All function calls appearing in expressions are external function calls. Such function could be an MSVL user-defined or external function. However it must satisfy that the function does not change any memory units or any external variables whose scopes are not limited to the function. Let $\sigma^{\prime}=\left\langle s_{0}^{\prime}, \ldots, s_{\left|\sigma^{\prime}\right|}^{\prime}\right\rangle$ be an interval over which the function is executed and $s_{0}^{\prime}=s_{i}$.

(1) $f$ is an MSVL user-defined function defined as follows:

$$
\text { function } f\left(\tau_{1} v_{1}, \ldots, \tau_{k} v_{k}, \tau R V a l\right)\{\phi\}
$$

The following is the evaluation rule of function call ext $f\left(r a_{1}, \ldots, r a_{k}, R V a l\right)$ :

$$
\begin{array}{ll} 
& \left(r a_{1}, \sigma_{i-1}, s_{i}, i\right) \Downarrow n_{1}, \ldots,\left(r a_{k}, \sigma_{i-1}, s_{i}, i\right) \Downarrow n_{k}, \\
\text { R10 } \quad \frac{\left(\phi \wedge \bigwedge_{j=1}^{k} v_{j} \Leftarrow n_{j}, \epsilon, s_{0}^{\prime}, 0\right) \stackrel{*}{\rightarrow}\left(\text { true }, \sigma^{\prime}, \emptyset,\left|\sigma^{\prime}\right|+1\right)}{\left(\operatorname{ext} f\left(r a_{1}, \ldots, r a_{k}, R V a l\right), \sigma_{i-1}, s_{i}, i\right) \Downarrow s_{\left|\sigma^{\prime}\right|}^{\prime r}(R V a l)}
\end{array}
$$

(2) $g$ is an external function and the evaluation rule of ext $g\left(r a_{1}, \ldots, r a_{k}\right)$ is given as follows:

$$
\mathrm{R} 11 \quad \frac{\left(r a_{1}, \sigma_{i-1}, s_{i}, i\right) \Downarrow n_{1}, \ldots,\left(r a_{k}, \sigma_{i-1}, s_{i}, i\right) \Downarrow n_{k}}{\left(\operatorname{ext} g\left(r a_{1}, \ldots, r a_{k}\right), \sigma_{i-1}, s_{i}, i\right) \Downarrow g\left(n_{1}, \ldots, n_{k}\right)}
$$

Semantic equivalence rules of function calls in statements

(1) The execution of an internal call of a user-defined MSVL function in statements is actually substituted by the execution of the body of the function with the arguments. $f$ is an MSVL user-defined function defined as follows:

$$
\text { function } f\left(\left(\tau_{j} v_{j},\right)^{*}(\tau R V a l)^{?}\right)\{m d c l ; m s\}
$$

where $m d c l$ is a list of declarations $\left(m d c l=(m P d ;)^{*}\right) ;(\tau R V a l)^{?}$ denotes an optional occurrence of $\tau R V a l$. The semantic equivalence rule of internal call statement $f\left(\left(r a_{j},\right)^{*}(R V a l)^{?}\right)$ is given as follows:

$$
\begin{aligned}
& \text { FUN } f\left(\left(r a_{j},\right)^{*}(R V a l)^{?}\right) \equiv\left(\left(\tau_{j} v_{j} \Leftarrow r a_{j} \wedge\right)^{*} m d c l\right) ; m s \text {; } \\
& \bigcirc\left(\text { ext } m \text { free }\left(\left(v_{j},\right)^{*}(R V a l,)^{?} m d c l\right) \wedge \text { empty }\right)
\end{aligned}
$$

where $m f r e e\left(\left(v_{j},\right)^{*}(R V a l,)^{?} m d c l\right)$, which is an external function, releases the memory of variables $v_{j}$ and $R V a l$, and variables declared in $m d c l$. 
(2) For the execution of each external function call in an MSVL program, only the information upon the beginning and ending points of the execution of the callee function is kept.

1) For any MSVL user-defined function, rule EXT1 of the external function call ext $f\left(\left(r a_{j},\right)^{*}(R V a l)^{?}\right)$ is given as follows:

EXT1. If $\sigma^{\prime}=\left\langle s_{0}^{\prime}, \ldots, s_{n}^{\prime}\right\rangle, s_{0}^{\prime}=s_{i}$ and $\left(\left(\left(\tau_{j} v_{j} \Leftarrow r a_{j} \wedge\right)^{*} m d c l\right) ; m s, \epsilon, s_{0}^{\prime}, 0\right) \stackrel{+}{\rightarrow}$ (true, $\left.\sigma^{\prime}, \emptyset, n+1\right)$, then $\left(\wedge\left\{\bigcirc p\right.\right.$, ext $\left.\left.f\left(\left(r a_{j},\right)^{*}(R V a l)^{?}\right)\right\}, \sigma_{i-1}, s_{i}, i\right) \rightarrow(p$, $\left.\sigma_{i}, s_{i+1}, i+1\right)$ and $s_{i+1}=s_{n}^{\prime}$.

2) For any call of external functions, the function has been actually parameterized with arguments, which can be regarded as the initial state of executing the function. Each assignment in the external function could be considered as the cause of state transitions. By executing the function, a finite state sequence can be generated and it is called a model of the function call. Then rules EXT2 and EXT3 of ext $g\left(r a_{1}, \ldots, r a_{k}\right)$ are given as follows:

EXT2. If for each $1 \leq j \leq k,\left(r a_{j}, \sigma_{i-1}, s_{i}, i\right) \Downarrow n_{j}$ and $\left\langle s_{i}\right\rangle$ is a model of $g\left(n_{1}, \ldots, n_{k}\right)$, then $\left(\wedge\left\{\bigcirc p\right.\right.$, ext $\left.\left.g\left(r a_{1}, \ldots, r a_{k}\right)\right\}, \sigma_{i-1}, s_{i}, i\right) \rightarrow\left(p, \sigma_{i}, s_{i+1}, i+\right.$ $1)$ and $s_{i+1}=s_{i}$. That means $g\left(r a_{1}, \ldots, r a_{k}\right)$ executes at a single state $s_{i}$, and state $s_{i}$ is inserted and concatenated with the main interval $\sigma_{i}$.

EXT3. If for each $1 \leq j \leq k,\left(r a_{j}, \sigma_{i-1}, s_{i}, i\right) \Downarrow n_{j}, s_{0}^{\prime}=s_{i}$ and $\sigma^{\prime}=\left\langle s_{0}^{\prime}, \ldots, s_{n}^{\prime}\right\rangle$ is a model of $g\left(n_{1}, \ldots, n_{k}\right)$, then $\left(\wedge\left\{\bigcirc p\right.\right.$, ext $\left.\left.g\left(r a_{1}, \ldots, r a_{k}\right)\right\}, \sigma_{i-1}, s_{i}, i\right) \rightarrow$ $\left(p, \sigma_{i}, s_{i+1}, i+1\right)$ and $s_{i+1}=s_{n}^{\prime}$. That means $g\left(r a_{1}, \ldots, r a_{k}\right)$ executes over an interval $\sigma^{\prime}$, and the final state $s_{n}^{\prime}$ over $\sigma^{\prime}$ is inserted and concatenated with the main interval $\sigma_{i}$.

\section{Translation from $\mathrm{Xd}-\mathrm{C}$ to MSVL}

In this section, an algorithm for translating an Xd-C program to an MSVL program is presented. Further, an example is given to show how the algorithm works.

\subsection{Translation Algorithm}

An Xd-C program is composed of a list of declarations $(P d ;)^{*}$ and functions (funct; )* (including main function). Thus, as shown in Algorithm 4.1, translating an Xd-C program to an MSVL program is actually translating declarations and functions from Xd-C to MSVL.

Algorithm 4.2 translates Xd-C declarations to MSVL declarations. For a variable declaration $\tau$ varlist, varlist is translated to an MSVL variable list using $\operatorname{VLTr}\left(\right.$ varlist). For an array initialization $\tau i d_{c}[]=\left\{(e,)^{*} e\right\}, \operatorname{count}\left((e,)^{*} e\right)$ is used to count the number of elements in array $i d_{c}$ and each element $e$ is translated to an MSVL expression by $\operatorname{ExTr}(e)$. For initialization of an array whose number of elements is specified, we just need to translate each element $e$ to an MSVL expression by $\operatorname{Ex} \operatorname{Tr}(e)$. For a structure definition struct $i d_{c 1}\left\{\left(\tau i d_{c 2} ;\right)^{*} \tau i d_{c 2}\right\}$, and is used to replace ';'. 

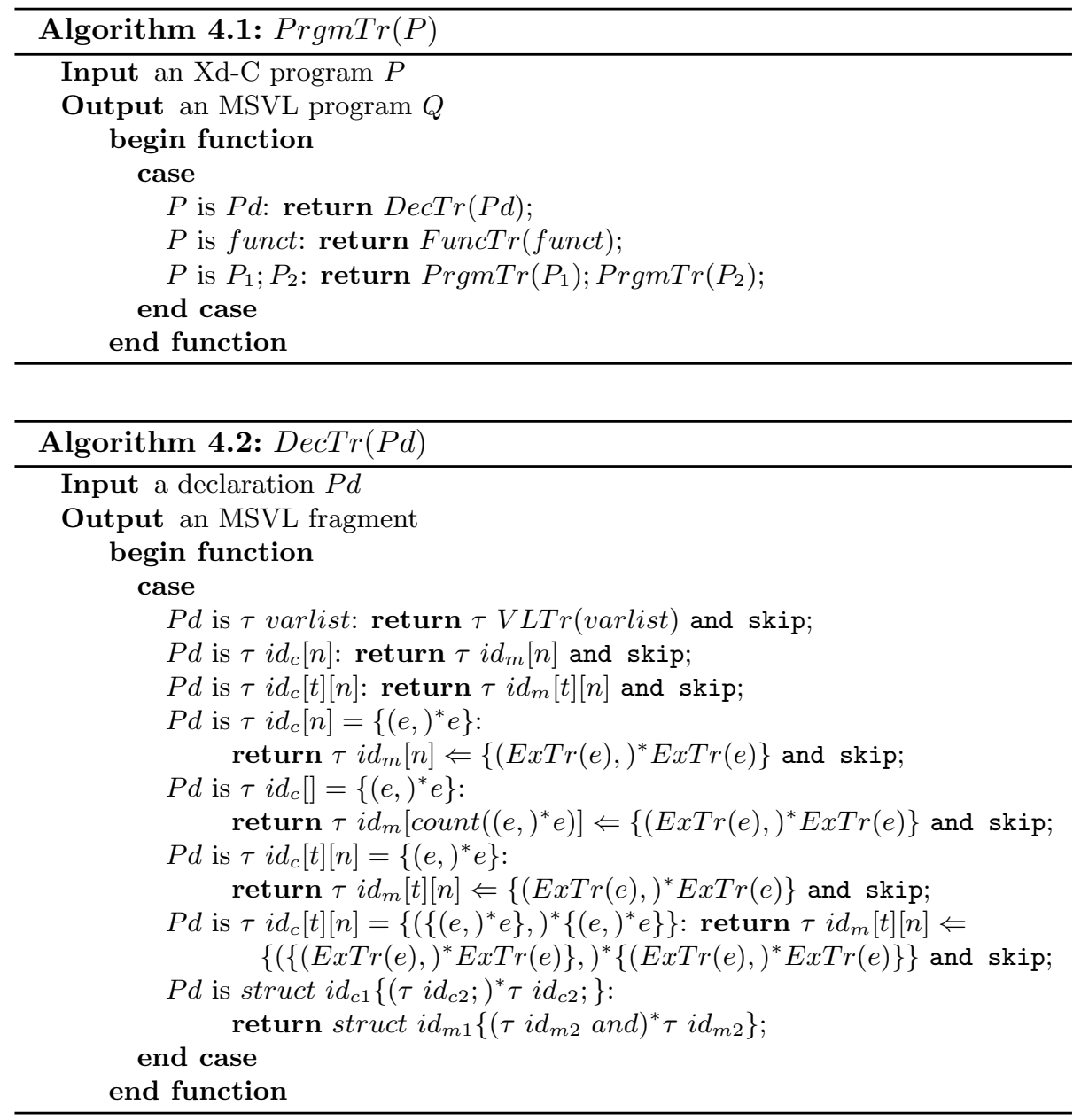

Algorithm 4.3 translates a variable list Varlst from Xd-C to MSVL. A variable $i d_{c}$ is directly translated to variable $i d_{m}$ in MSVL and a variable initialization $i d_{c}=e$ is translated to $i d_{m} \Leftarrow \operatorname{ExTr}(e)$.

Algorithm 4.4 shows how to translate each expression from Xd-C to MSVL. A constant $c$ and a variable $i d_{c}$ can directly be translated to $c$ and $i d_{m}$ in MSVL while for other expressions such as $i d_{c}[e], x\left(e_{1}, \ldots, e_{k}\right),(\tau) e, o p_{1} e$ and $e_{1}$ op $e_{2}$, sub-expressions $e_{1}, e_{2}, \ldots, e_{k}, e$ and $x$ are translated to their corresponding MSVL expressions. Operations $==, \& \&$ and $\|$ are translated to $=$, and and or, respectively. Note that since a toolkit can only recognize strings of ordinary symbols, we replace $\neg, \wedge$ and $\vee$ by !, and and or, respectively. Expression $e_{1} ? e_{2}: e_{3}$ is translated to if $\left(\operatorname{Ex} \operatorname{Tr}\left(e_{1}\right)\right)$ then $\operatorname{Ex} \operatorname{Tr}\left(e_{2}\right)$ else $\operatorname{Ex} \operatorname{Tr}\left(e_{3}\right)$.

A function funct can be translated to an MSVL function using Algorithm 4.5. It translates variable declarations and statements from Xd-C to MSVL by 

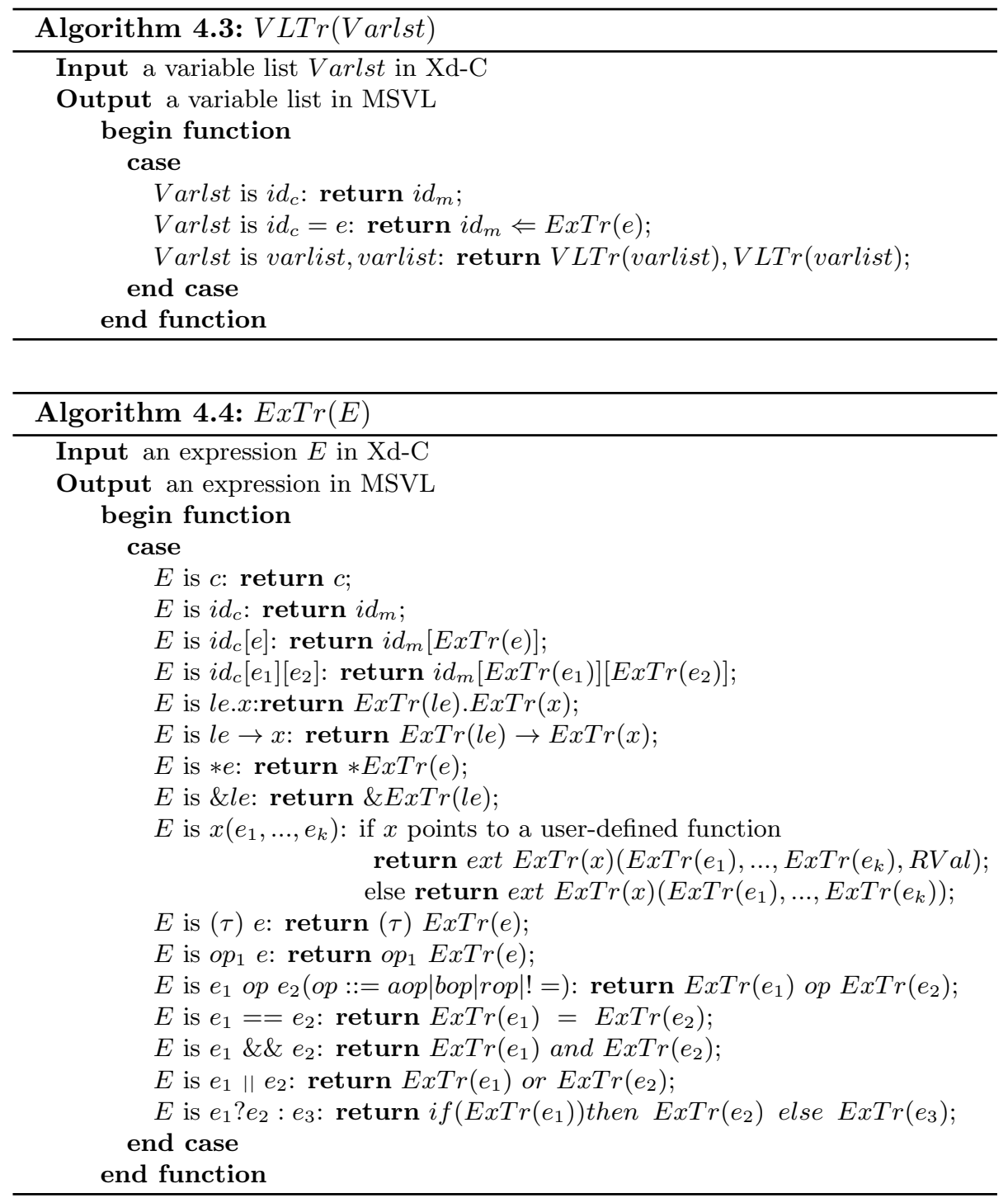

means of DecTr and StmtTr, respectively. Algorithm4.6 is presented to translate each Xd-C statement to an MSVL statement. A null statement is translated to MSVL statement empty. Simple assignment $l e=e$, post increment le++ and post decrement $l e--$ are translated to MSVL unit assignment statements $\operatorname{ExTr}(l e):=\operatorname{Ex} \operatorname{Tr}(e), \operatorname{Ex} \operatorname{Tr}(l e):=\operatorname{Ex} \operatorname{Tr}(l e)+1$ and $\operatorname{ExTr}(l e):=\operatorname{Ex} \operatorname{Tr}(l e)-$ 1 , respectively. A conditional statement is translated to MSVL conditional statement if $(E x \operatorname{Tr}(e))$ then $\left\{\operatorname{Stmt} \operatorname{Tr}\left(c s_{1}\right)\right\}$ else $\left\{\operatorname{Stmt} \operatorname{Tr}\left(c s_{2}\right)\right\}$. Sequential, switch, while loop, do loop and for loop statements are translated to MSVL statements by Algorithms ChopTr, SwitchTr, WhileTr, DoTr and ForTr, respectively. 


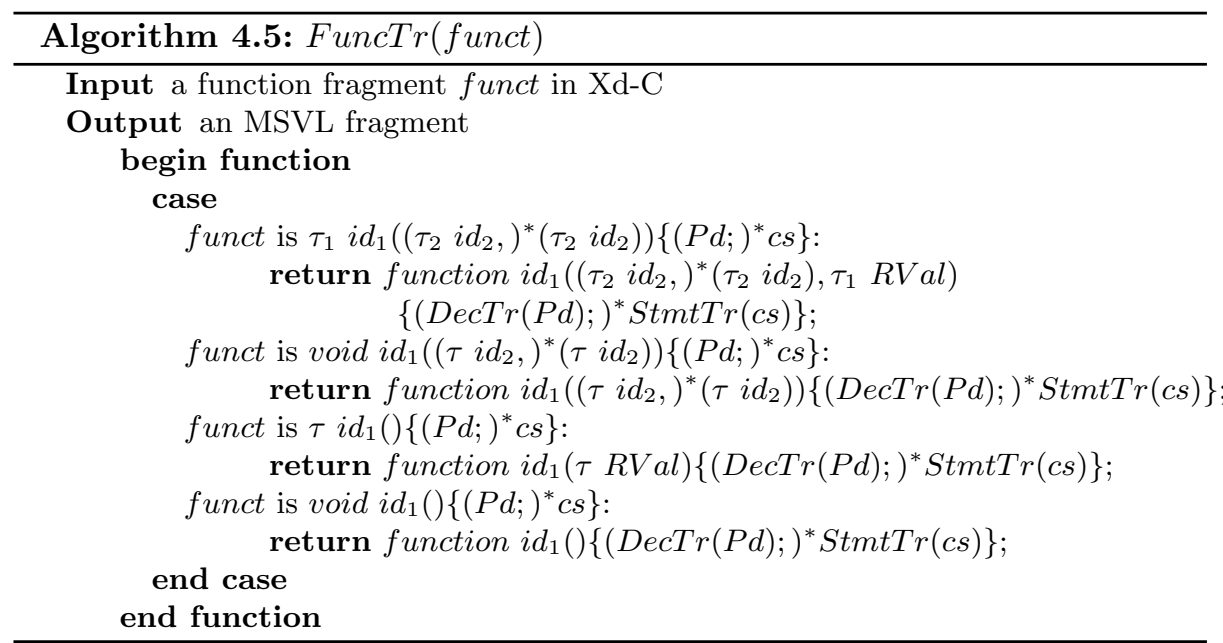

In order to translate continue, break and return in an Xd-C program to MSVL statements, variables continue, break and return are introduced as key variables in MSVL to handle continue, break and return statements in the $\mathrm{Xd}-\mathrm{C}$ program. The translation of a function call statement " $x\left(e_{1}, \ldots, e_{k}\right)$;" is divided into three cases:

(1) For a function call of a user-defined function without a return value, all sub-expressions $e_{1}, \ldots, e_{k}$ and $x$ are translated to MSVL expressions by ExTr.

(2) For a function call of a user-defined function with a return value, an extra argument $R V a l$ storing the return value of the function call is introduced.

(3) For a function call of an external function, a key word ext is added before the function call which represents the function call is an external call.

A sequential statement " $c s_{1} ; c s_{2}$;" is translated to an MSVL statement by Algorithm 4.7 in four cases. If there is no break, return or continue in $c s_{1}$, sub-statements $c s_{1}$ and $c s_{2}$ are translated to MSVL statements using StmtTr. If there is break, return or continue in $c s_{1}$, the sequential statement is translated to "Stmt $\operatorname{Tr}\left(c s_{1}\right)$;if $($ break $=0)$ then $\left\{\operatorname{Stmt} \operatorname{Tr}\left(c s_{2}\right)\right\}$ else $\{$ empty\}", "Stmt $\operatorname{Tr}\left(c s_{1}\right) ;$ if $($ return $=0)$ then $\left\{\operatorname{Stmt} \operatorname{Tr}\left(c s_{2}\right)\right\}$ else\{empty\}" or "StmtTr( $\left.c s_{1}\right)$; if $($ continue $=0)$ then $\left\{\operatorname{Stmt} \operatorname{Tr}\left(c s_{2}\right)\right\}$ else $\{$ empty $\} "$.

Algorithm 4.8 translates each case of a switch statement to an MSVL conditional statement. For a switch statement, if the value of $e$ is $n$ and no break or return statement occurs before it, case $n: c s$ is chosen to execute; for the case following case $n$ (including the default case), if there is no break or return before it, the case is also executed.

Algorithm 4.9 translates a while loop statement while $(e)\{c s\}$ to an MSVL statement according to whether there is break, return or continue. If there 

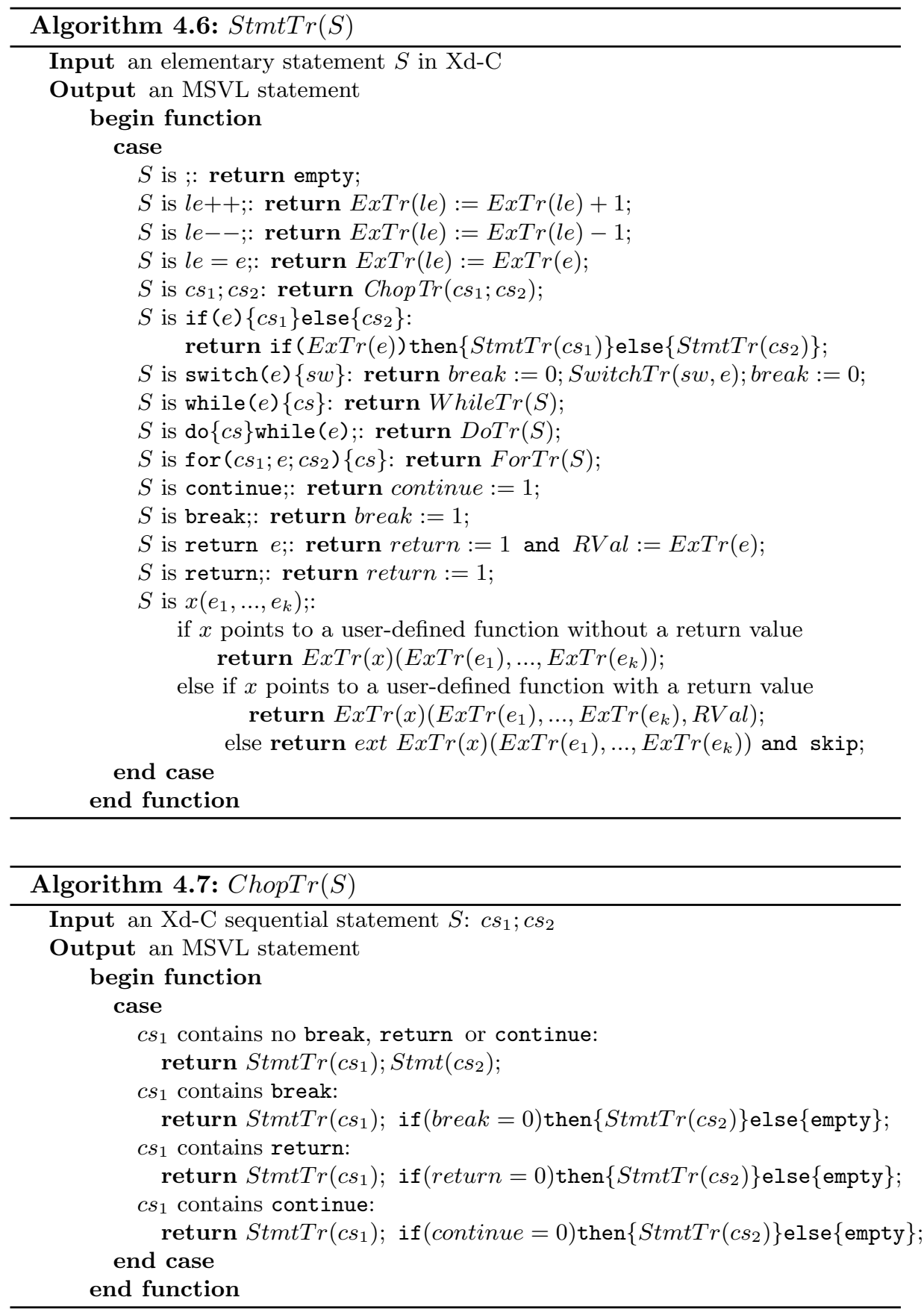

is no break, return or continue statement in $c s$, it is directly translated to a 
while statement in MSVL. If there is break or return in $c s$, the value of break or return should also be concerned in the condition of the while statement. If there is a continue statement in $c s$, the value of continue should be set to 0 at the end of each loop. The translation procedures of do loop and for loop statements are similar to the translation of while loop and are shown in Algorithm 4.10 and 4.11 , respectively.
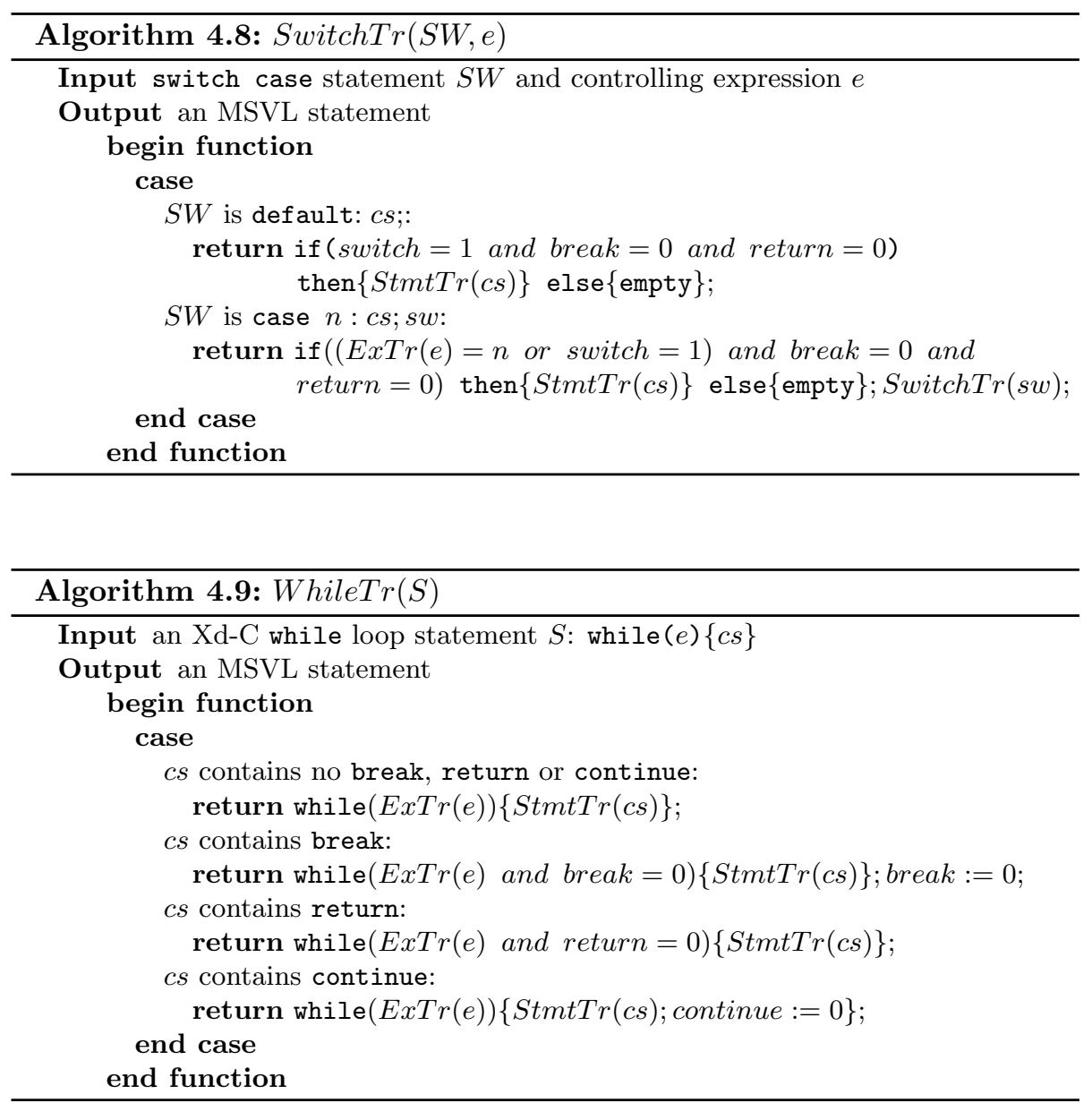

\subsection{An Example}

In this section, an application bzip2 [29] is used to show how an Xd-C program is translated to an MSVL program. bzip2 is a compression program to compress and decompress input files. As shown in Fig, 2 , the left-hand side is the core of a function generateMTFValues in bzip2, including most kinds of Xd-C statements and the right-hand side is the translated MSVL program by using 

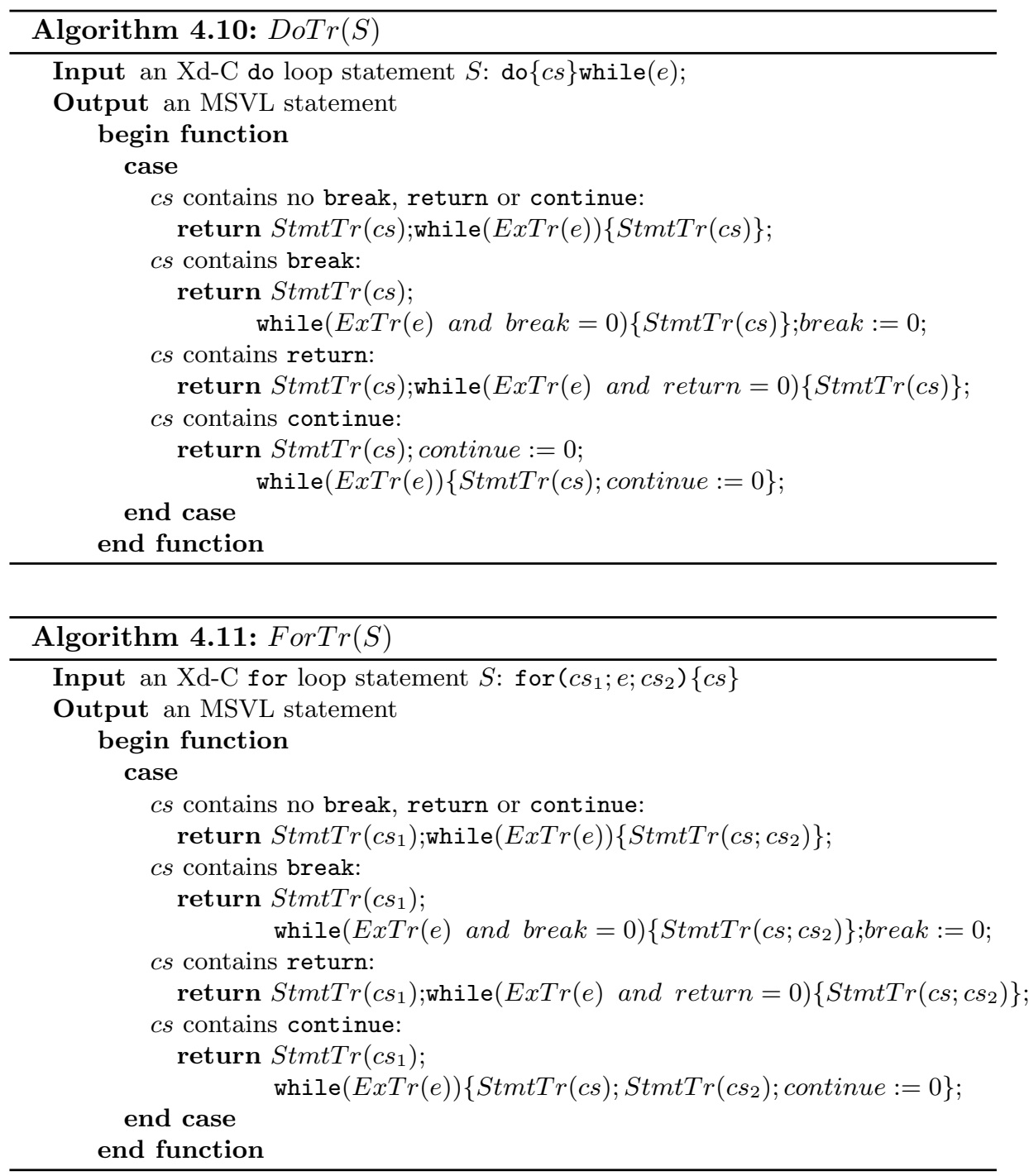

the translation algorithms. Various kinds of Xd-C statements in the program are translated to their equivalent MSVL statements as follows:

(1) Function definition statement void generateMTFValues ()$\{\ldots\}$ can directly be translated to MSVL function definition statement function generateMTFValues ()$\{\ldots\}$.

(2) Variable declaration statement "unsigned char yy[256];" is translated to unsigned char $y y[256]$ and skip.

(3) Simple assignment statement " $i=0$;" is directly translated to MSVL unit 


\section{Proof of Equivalence}

Since the types in MSVL are the same as in Xd-C fragments, we only need to prove the equivalence between expressions and statements involved in $\mathrm{Xd}-\mathrm{C}$ and MSVL programs.

In order to account for differences in allocation patterns between Xd-C and MSVL programs, a function $\alpha$ is used to denote a memory injection 32. It is an injective function from Xd-C block reference $b$ to $\left(b^{\prime}, \delta^{\prime}\right)$, which means that block $b$ in the Xd-C memory state corresponds to block $b^{\prime}$ and offset $\delta^{\prime}$ in the MSVL memory state.

Based on a memory injection $\alpha$, an equivalence relation between an Xd-C value $v$ and an MSVL value $n$, written by $\alpha \vdash v \sim n$, is defined as follows:

(1) $\alpha \vdash c \sim c$, where $v=n=c$.

(2) $\quad \alpha \vdash p \operatorname{tr}(b, i) \sim \operatorname{ptr}\left(b^{\prime}, i^{\prime}\right)$, where $v=p \operatorname{tr}(b, i)$ and $n=p \operatorname{tr}\left(b^{\prime}, i^{\prime}\right)$, if and only if there exists $\delta \in N_{0}$ such that $\alpha(b)=\left(b^{\prime}, \delta\right)$ and $i^{\prime}=i+\delta$.

Rule (1) means that a constant $c$ in Xd-C is equivalent to $c$ in MSVL. For example, $\alpha \vdash 1 \sim 1$ and $\alpha \vdash 1.2 \sim 1.2$. Rule $(2)$ means that a pointer value $\operatorname{ptr}(b, j)$ in $\mathrm{Xd}-\mathrm{C}$ is equivalent to a pointer value $\operatorname{ptr}\left(b^{\prime}, j^{\prime}\right)$ in MSVL. For example, we assume the location of a variable $x$ in an $\mathrm{Xd}-\mathrm{C}$ program is $(0 x f f f 0000,0)$. If $\alpha(0 x f f f f 0000)=(0 x f f f f a a a a, 8)$ which indicates that an Xd-C block 0xffff0000 corresponds to an MVSL block 0xffffaaaa and offset 8 , we have $\alpha \vdash \operatorname{ptr}(0 \mathrm{xffff0000,0)} \sim \operatorname{ptr}(0 \mathrm{xffffaaaa}, 8)$.

Lemma 1. For a given $\alpha$, any $b, b^{\prime} \in \mathbb{Z}$ and $i, i^{\prime}, j, j^{\prime} \in N_{0}$, if $\alpha \vdash p \operatorname{tr}(b, i) \sim$ $\operatorname{ptr}\left(b^{\prime}, i^{\prime}\right)$ and $j=j^{\prime}$, then $\alpha \vdash p \operatorname{tr}(b, i+j) \sim \operatorname{ptr}\left(b^{\prime}, i^{\prime}+j^{\prime}\right)$.

\section{Proof:}

$$
\begin{aligned}
& (1) \quad \alpha \vdash p \operatorname{tr}(b, i) \sim p \operatorname{tr}\left(b^{\prime}, i^{\prime}\right) \wedge j=j^{\prime} \\
& (2) \Longrightarrow \alpha(b)=\left(b^{\prime}, \delta\right) \wedge i^{\prime}=i+\delta \wedge j=j^{\prime} \\
& (3) \Longrightarrow \alpha(b)=\left(b^{\prime}, \delta\right) \wedge i^{\prime}+j^{\prime}=i+j+\delta \\
& (4) \Longrightarrow \quad \Longrightarrow \vdash p \operatorname{tr}(b, i+j) \sim p \operatorname{tr}\left(b^{\prime}, i^{\prime}+j^{\prime}\right)
\end{aligned}
$$

Let $\mathbb{M}$ denote the set of all memory states $M$ in Xd-C programs and $\mathbb{S}$ the set of all states $s$ in MSVL programs. The equivalences of states, expressions and statements are respectively defined as follows.

Definition 1. (State Equivalence) For a given memory injection $\alpha$, an $X d-C$ memory state $M$ is equivalent to an MSVL state $s$, denoted by $\alpha \vdash M \sim s$, if and only if the following condition holds:

For any variable $x_{c} \in \operatorname{Dom}(G \cup E)$ in an $X d$-C program, the corresponding variable $x_{m} \in \operatorname{Dom}(s) \backslash\{$ break, continue, return, RVal $\}$ in an MSVL program, $b, b^{\prime} \in \mathbb{Z}, j, j^{\prime} \in N_{0}$ and $v, n \in D$, if $\left(G, E \vdash x_{c}, M \stackrel{l}{\Rightarrow}(b, j)\right)$ and $(G, E \vdash$ $\left.x_{c}, M \Rightarrow v\right)$ in the $X d$-C program, as well as $s^{l}\left(x_{m}\right)=\left(b^{\prime}, j^{\prime}\right)$ and $s^{r}\left(x_{m}\right)=n$ in the MSVL program, then $\alpha \vdash p \operatorname{tr}(b, j) \sim \operatorname{ptr}\left(b^{\prime}, j^{\prime}\right)$ and $\alpha \vdash v \sim n$. 
Definition 1 defines the equivalence between states $M$ and $s$. Intuitively, $M$ is equivalent to $s$ means that for each variable in both Xd-C and MSVL programs, the locations and values of the variable are equivalent, respectively. The following is an example of state equivalence.

Example 1. In an Xd-C program, there are two declared variables $x_{c}$ and $y_{c}$. At state $M$, the location of $x_{c}$ is $(0 x f f f f 0000,0)$ with value 1 , while the location of $y_{c}$ is $(0 x f f f f 1111,0)$ with value $\operatorname{ptr}(0 \mathrm{xffff} 0000,0)$. That is, $x_{c}=1$ and $y_{c}=\& x_{c}$. In the corresponding MSVL program, $x_{m}$ and $y_{m}$ are also declared variables. At state $s$, the location of $x_{m}$ is $(0 \mathrm{xffffaaaa}, 0)$ with value 1 , while the location of $y_{m}$ is $(0 x f f f f 3333,0)$ with value $\operatorname{ptr}(0 \mathrm{xffffaaaa}, 0)$. We also have $x_{m}=1$ and $y_{m}=\& x_{m}$. Thus, for a given memory injection $\alpha$ satisfying $\alpha(0 x f f f f 0000)=(0 x f f f f a a a a, 0)$ and $\alpha(0 x f f f f 1111)=(0 x f f f 3333,0)$, we have $\alpha \vdash$ $p \operatorname{tr}(0 x \mathrm{xfff} 0000,0) \sim \operatorname{ptr}(0 \mathrm{xffffaaaa}, 0), \alpha \vdash 1 \sim 1$ and $\alpha \vdash p \operatorname{tr}(0 \mathrm{xffff1111,0}) \sim$ $p \operatorname{tr}(0 x f f f f 3333,0)$. Therefore, the locations and values of the two variables between $M$ and $s$ are equivalent, respectively. Hence, $\alpha \vdash M \sim s$.

Definition 2. (Left-value Expression Equivalence) For a given memory injection $\alpha$, an $X d$ - $C$ left-value expression $e$ is equivalent to an MSVL left-value expression $a$, denoted by $\alpha \vdash e \sim_{l} a$, if and only if for any $M, s, b, j, b^{\prime}$, $j^{\prime}$ and $\sigma$, if $\alpha \vdash M \sim s,(G, E \vdash e, M \stackrel{l}{\Rightarrow}(b, j))$ in the $X d-C$ program and $(a, \sigma, s,|\sigma|+1) \stackrel{l}{\Rightarrow}\left(b^{\prime}, j^{\prime}\right)$ in the MSVL program, then $\alpha \vdash \operatorname{ptr}(b, j) \sim \operatorname{ptr}\left(b^{\prime}, j^{\prime}\right)$.

Intuitively, the equivalence between left-value expressions is merely that the equivalence of expression locations in Xd-C and MSVL. Further, if the locations of expressions are equivalent, the expressions stand for the same variable and their values are equivalent. For instance, the following example illustrates the situation.

Example 2. As in Example 1, in an Xd-C program, $* y_{c}$ is a left-value expression and $\left(G, E \vdash * y_{c}, M \stackrel{l}{\Rightarrow}(0 x f f f f 0000,0)\right)$, while in the corresponding MSVL program, $\left(* y_{m}, \sigma, s,|\sigma|+1\right) \stackrel{l}{\Rightarrow}(0 x$ ffffaaaa, 0$)$. Since $\alpha \vdash p \operatorname{tr}(0 \mathrm{xffff} 0000,0) \sim$ $\operatorname{ptr}(0 x f f f f a a a a, 0)$, we have $\alpha \vdash * y_{c} \sim_{l} * y_{m}$. Here, $* y_{c}$ and $* y_{m}$ stand for variables $x_{c}$ and $x_{m}$, respectively. In fact, the values of $* y_{c}$ and $* y_{m}$ are equivalent.

Definition 3. (Right-value Expression Equivalence) For a given memory injection $\alpha$, an $X d-C$ right-value expression $e$ is equivalent to an MSVL right-value expression $a$, denoted by $\alpha \vdash e \sim_{r} a$, if and only if for any $M, s, v, n$ and $\sigma$, if $\alpha \vdash M \sim s,(G, E \vdash e, M \Rightarrow v)$ in the Xd-C program and $(a, \sigma, s,|\sigma|+1) \Downarrow n$ in the MSVL program, then $\alpha \vdash v \sim n$.

As a matter of fact, the equivalence between right-value expressions is really the equivalence between the expression values in Xd-C and MSVL under the condition of state equivalence. The following is an example of the equivalence relation.

Example 3. In an Xd-C program, $\left(x_{c}>0\right) ? 2: 3$ is a right-value expression with $x_{c}=1$, hence, $\left(G, E \vdash\left(x_{c}>0\right) ? 2: 3, M \Rightarrow 2\right)$. Whereas in the corresponding MSVL program, $x_{m}=1$ and $\left(\right.$ if $\left(x_{m}>0\right)$ then 2 else $\left.3, \sigma, s,|\sigma|+1\right) \Downarrow 2$. Since $\alpha \vdash 2 \sim 2$, we have $\alpha \vdash\left(x_{c}>0\right) ? 2: 3 \sim_{r}$ if $\left(x_{m}>0\right)$ then 2 else 3 . 
Definition 4. (Expression Equivalence) For a given memory injection $\alpha$, an $X d-C$ expression e is equivalent to an MSVL expression a, denoted by $\alpha \vdash e \sim_{e}$ $a$, if and only if either $e$ and $a$ are both left-value expressions and $\alpha \vdash e \sim_{l} a$, or $e$ and $a$ are both only right-value expressions and $\alpha \vdash e \sim_{r} a$.

Definition 5. (Terminating Statement Equivalence) For a given memory injection $\alpha$, a terminating statement cs in an $X d-C$ program is equivalent to a terminating statement $m s$ in an $M S V L$ program, denoted by $\alpha \vdash c s \sim_{t} m s$, if and only if for any $M, s_{i}$, out and $M^{\prime}$, if $\alpha \vdash M \sim s_{i}$ and $\left(G, E \vdash c s, M \stackrel{t}{\Rightarrow}\right.$ out,$\left.M^{\prime}\right)$ in the $X d$ - $C$ program, then there exists $\sigma \in \Gamma$ such that $\left(m s, \sigma_{i-1}, s_{i}, i\right) \stackrel{*}{\rightarrow}$ (true, $\sigma, \emptyset,|\sigma|+1)$ in the $M S V L$ program and $\alpha \vdash M^{\prime} \sim s_{|\sigma|}$.

The equivalence between terminating statements in Xd-C and MSVL indicates that if the initial states are equivalent, then after executing the programs, the final states are equivalent. For instance, Example \& shows the equivalence relation between Xd-C and MSVL statements.

Example 4. As in Example 1, in an Xd-C program, " $x_{c}=2$;" is a terminating statement and $\left(G, E \vdash x_{c}=2 ;, M \Rightarrow\right.$ out,$\left.M^{\prime}\right)$. At state $M^{\prime}$, the value of $x_{c}$ is changed to 2. The locations of $x_{c}$ and $y_{c}$, and the value of $y_{c}$ are not changed. Whereas in the corresponding MSVL program, $s_{i}=s$ and $\left(x_{m}:=\right.$ $\left.2, \sigma_{i-1}, s_{i}, i\right) \stackrel{*}{\rightarrow}($ true $, \sigma, \emptyset,|\sigma|+1)$. At state $s_{|\sigma|}, s_{|\sigma|}^{r}\left(x_{m}\right)=2, s_{|\sigma|}^{l}\left(x_{m}\right)=$ $s_{i}^{l}\left(x_{m}\right), s_{|\sigma|}^{l}\left(y_{m}\right)=s_{i}^{l}\left(y_{m}\right)$ and $s_{|\sigma|}^{r}\left(y_{m}\right)=s_{i}^{r}\left(y_{m}\right)$. Hence, $\alpha \vdash M^{\prime} \sim s_{|\sigma|}$. Consequently, we have $\alpha \vdash x_{c}=2 ; \sim_{t} x_{m}:=2$.

Definition 6. ( $X d-C$ Statement Equivalence) An $X d-C$ statement cs is equivalent to $c s^{\prime}$ executed from state $M$, denoted by $(c s, M) \cong\left(c s^{\prime}, M\right)$, if and only if two intervals $M_{\sigma}=\left(M_{0}, M_{1}, \ldots\right)$ and $M_{\sigma}^{\prime}=\left(M_{0}^{\prime}, M_{1}^{\prime}, \ldots\right)$ generated by respectively executing $\mathrm{cs}$ and $c s^{\prime}$ from $M$ are equivalent, that is, $M_{i}=M_{i}^{\prime}$ for $i \geq 0$.

Lemma 2. (C_loop) In an Xd-C program, if $(G, E \vdash e, M \Rightarrow$ true $)$ and $(G, E \vdash$ $c s, M \stackrel{t}{\Rightarrow}$ Normal, $\left.M_{1}\right)$, then (while $\left.(e)\{c s\}, M\right) \cong(c s ;$ while $(e)\{c s\}, M)$.

Proof: Suppose $M_{\sigma}$ and $M_{\sigma}^{\prime}$ are generated by executing while $(e)\{c s\}$ and "cs; while $(e)\{c s\}$ " from $M$, respectively. Since $(G, E \vdash e, M \Rightarrow t r u e)$ and $(G, E \vdash$ $c s, M \stackrel{t}{\Rightarrow} N$ ormal, $M_{1}$ ), a prefix $\left(M, M_{1}\right)$ (rule $\left.T 13\right)$ of $M_{\sigma}$ is generated while the same prefix $\left(M, M_{1}\right)$ (rule $\left.T 7\right)$ of $M_{\sigma}^{\prime}$ is also generated. Therefore, $M_{\sigma}$ and $M_{\sigma}^{\prime}$ are equivalent. According to Definition 6 $(\operatorname{while}(e)\{c s\}, M) \cong(c s ; \operatorname{while}(e)$ $\{c s\}, M)$.

Definition 7. (Diverging Statement Equivalence) For a given memory injection $\alpha$, a diverging statement $c s$ in an $X d-C$ program is equivalent to a diverging statement $m s$ in an MSVL program, denoted by $\alpha \vdash c s \sim_{d} m s$, if and only if for any $M$ and $s_{i}$, if $\alpha \vdash M \sim s_{i}$ and $(G, E \vdash c s, M \stackrel{T}{\Rightarrow} \infty)$ in the 
$X d-C$ program, then cs at state $M$ and $m s$ at state $s_{i}$ can both be rewritten as an infinite sequence of terminating statements $(c s, M) \cong\left(c s_{1} ; c s_{2} ; \ldots, M\right)$ and $\left(m s, \sigma_{i-1}, s_{i}, i\right) \stackrel{*}{\longmapsto}\left(m s_{1} ; m s_{2} ; \ldots, \sigma_{i-1}, s_{i}, i\right)$, and $\alpha \vdash c s_{j} \sim_{t} m s_{j}$ for all $j \geq 1$.

The following is an example of diverging statement equivalence.

Example 5. In an Xd-C program, the initial value of variable $x_{c}$ is 1 . "while $\left(x_{c}>0\right)\left\{x_{c}++;\right\}$ " is a diverging statement and (while $\left(x_{c}>0\right)\left\{x_{c}++;\right\}$, $M) \cong\left(x_{c}++; x_{c}++; \ldots, M\right)$. Whereas in the corresponding MSVL program, the initial value of variable $x_{m}$ is also 1 . (while $\left(x_{m}>0\right)\left\{x_{m}:=x_{m}+\right.$ $\left.1\}, \sigma_{i-1}, s_{i}, i\right) \stackrel{*}{\longmapsto}\left(x_{m}:=x_{m}+1 ; x_{m}:=x_{m}+1 ; \ldots, \sigma_{i-1}, s_{i}, i\right)$. Since $\alpha \vdash$ $x_{c}++; \sim_{t} x_{m}:=x_{m}+1$, we have $\alpha \vdash$ while $\left(x_{c}>0\right)\left\{x_{c}++;\right\} \sim_{d}$ while $\left(x_{m}>\right.$ $0)\left\{x_{m}:=x_{m}+1\right\}$.

Definition 8. (Statement Equivalence) For a given memory injection $\alpha$, a statement cs in an Xd-C program is equivalent to a statement ms in an MSVL program, denoted by $\alpha \vdash c s \sim_{s} m s$, if and only if either $\alpha \vdash c s \sim_{t} m s$ or $\alpha \vdash c s \sim_{d} m s$.

\subsection{Proof of expression equivalence}

Theorem 1. Suppose an $X d-C$ expression e is transformed to an $M S V L$ expression a by Algorithm 4.4 along with converting an $X d$-C program to an $M S V L$

program. That is, $a=\operatorname{Ex} \operatorname{Tr}(e)$. For a given $\alpha$, any $M \in \mathbb{M}$ and $s \in \mathbb{S}$, if $\alpha \vdash M \sim s$, then $\alpha \vdash e \sim_{e} a$.

Proof: The proof proceeds by induction on the structure of expressions.

Base:

1. For a constant $c$, the conclusion is trivially true.

2. For a variable $i d_{c}, \operatorname{Ex} \operatorname{Tr}\left(i d_{c}\right)=i d_{m}$. Here, $i d_{c}$ and $i d_{m}$ are left-value expressions.

$$
\begin{aligned}
& \text { (1) } \quad \alpha \vdash M \sim s \\
& \text { (2) } \Longrightarrow \quad \forall b, b^{\prime}, j, j^{\prime} .\left(G, E \vdash i d_{c}, M \stackrel{l}{\Rightarrow}(b, j)\right) \wedge s^{l}\left(i d_{m}\right)=\left(b^{\prime}, j^{\prime}\right) \\
& \rightarrow \alpha \vdash \operatorname{ptr}(b, j) \sim \operatorname{ptr}\left(b^{\prime}, j^{\prime}\right) \quad \text { Definition 1 (1) } \\
& \text { (3) } \quad\left(i d_{m}, \sigma, s,|\sigma|+1\right) \stackrel{l}{\Rightarrow} s^{l}\left(i d_{m}\right) \\
& \text { (4) } \Longrightarrow \quad \forall b, b^{\prime}, j, j^{\prime} .\left(G, E \vdash i d_{c}, M \stackrel{l}{\Rightarrow}(b, j)\right) \wedge\left(i d_{m}, \sigma, s,|\sigma|+1\right) \stackrel{l}{\Rightarrow}\left(b^{\prime}, j^{\prime}\right) \\
& \rightarrow \alpha \vdash \operatorname{ptr}(b, j) \sim \operatorname{ptr}\left(b^{\prime}, j^{\prime}\right) \\
& \text { (5) } \Longleftrightarrow \alpha \vdash i d_{c} \sim_{l} i d_{m} \quad \text { Definition 2 (4) } \\
& \text { (6) } \Longrightarrow \alpha \vdash i d_{c} \sim_{e} i d_{m}
\end{aligned}
$$

Induction:

3. For $i d_{c}[e]$ of type $\tau, \operatorname{Ex} \operatorname{Tr}\left(i d_{c}[e]\right)=i d_{m}[r a]$, where $r a=\operatorname{Ex} \operatorname{Tr}(e), i d_{c}$, $i d_{m}, i d_{c}[e]$ and $i d_{m}[\mathrm{ra}]$ are all left-value expressions while $e$ and $r a$ are 
both right-value expressions.

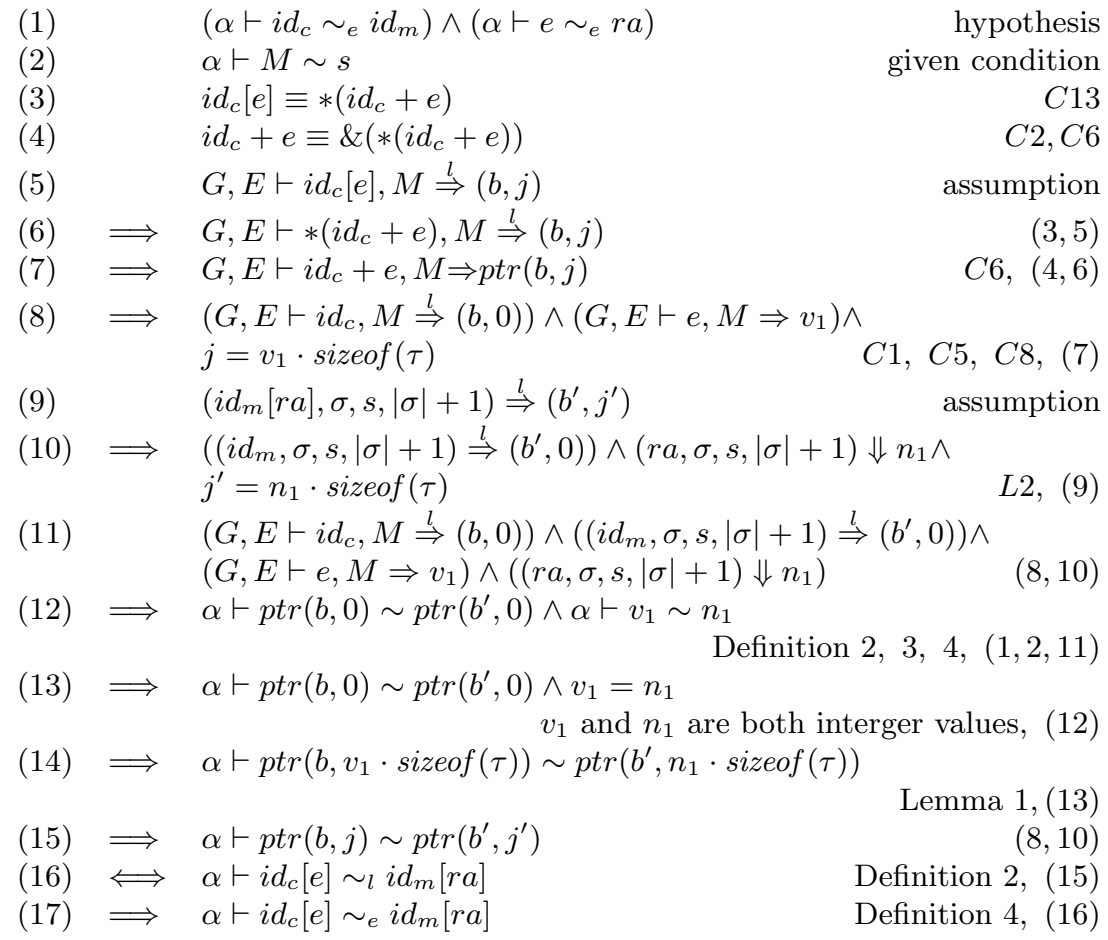

In a similar way, it can be proved that the conclusion is true for $i d_{c}\left[e_{1}\right]\left[e_{2}\right]$.

4. For le.x of type $\tau, \operatorname{ExTr}(l e . x)=l a . y$, where $l a=\operatorname{ExTr}(l e)$ and $y=$ $\operatorname{Ex} \operatorname{Tr}(x), l e, l a, l e . x$ and la.y are all left-value expressions. When translating a member $x$ of a struct variable to an expression in MSVL using ExTr, we do the following: if $x$ is the $k$ th member of a struct $S_{c}$ with filed list $\varphi$ in the $\mathrm{Xd}-\mathrm{C}$ program, it is translated to the $k$ th member of a struct $S_{m}$ with filed list $\varphi$ in the MSVL program. We assume $\tau_{i}$ is the type of $i$ th member of struct $S_{c}$ for $0 \leq i<k$. Therefore, field_offset $(x, \varphi)=$ field_offset $(y, \varphi)=\operatorname{sizeof}\left(\tau_{1}\right)+\ldots+\operatorname{sizeof}\left(\tau_{k-1}\right)$. For convenience, we denote $\delta^{\prime}=$ field_offset $(x, \varphi)$ in the following.

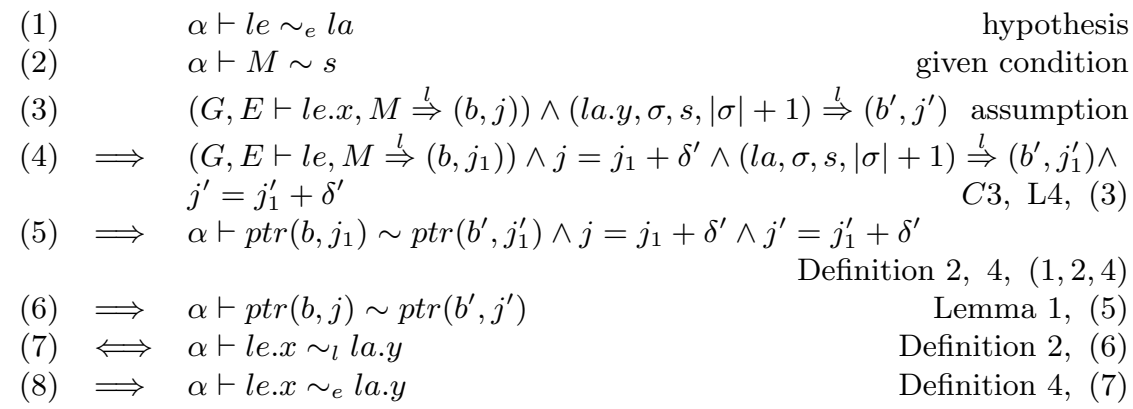

Similarly, we can prove that the conclusion is true for $l e \rightarrow x$. 
5. For $* e, E x \operatorname{Tr}(* e)=* p t$, where $p t=\operatorname{Ex} \operatorname{Tr}(e), * e$ and $* p t$ are both leftvalue expressions while $e$ and $p t$ are both right-value expressions.
(1) $\quad \alpha \vdash e \sim_{e} p t$
hypothesis
(2) $\quad \alpha \vdash M \sim s$
given condition
(3) $\quad e \equiv \&(* e) \wedge p t \equiv \&(* p t)$
$C 2, C 6$
(4) $\quad(G, E \vdash * e, M \stackrel{l}{\Rightarrow}(b, j)) \wedge(* p t, \sigma, s,|\sigma|+1) \stackrel{l}{\Rightarrow}\left(b^{\prime}, j^{\prime}\right) \quad$ assumption
$(5) \quad \Longrightarrow \quad(G, E \vdash e, M \Rightarrow p \operatorname{tr}(b, j)) \wedge(p t, \sigma, s,|\sigma|+1) \Downarrow p \operatorname{tr}\left(b^{\prime}, j^{\prime}\right)$
C6, R3, $(3,4)$
(6) $\Longrightarrow \quad \alpha \vdash p \operatorname{tr}(b, j) \sim p \operatorname{tr}\left(b^{\prime}, j^{\prime}\right)$
Definition $2,3,4,(1,2,5)$
(7) $\Longleftrightarrow \alpha \vdash * e \sim_{l} * p t$
(8) $\Longrightarrow \alpha \vdash * e \sim_{e} * p t$

6. For \&le, ExTr(\&le) $=\& l a$, where $l a=E x \operatorname{Tr}(l e)$, \&le and \&la are both right-value expressions while $l e$ and $l a$ are both left-value expressions.

(1) $\quad \alpha \vdash l e \sim_{e} l a$

(2) $\quad \alpha \vdash M \sim s$

hypothesis

$l e \equiv *(\& l e) \wedge l a \equiv *(\& l a)$

given condition

$(G, E \vdash \& l e, M \Rightarrow p \operatorname{tr}(b, j)) \wedge(\& l a, \sigma, s,|\sigma|+1) \Downarrow p \operatorname{tr}\left(b^{\prime}, j^{\prime}\right)$

$C 2, C 6$

$(5) \quad \Longrightarrow \quad(G, E \vdash l e, M \stackrel{l}{\Rightarrow}(b, j)) \wedge\left((l a, \sigma, s,|\sigma|+1) \stackrel{l}{\Rightarrow}\left(b^{\prime}, j^{\prime}\right)\right)$

(6) $\Longrightarrow \alpha \vdash p \operatorname{tr}(b, j) \sim \operatorname{ptr}\left(b^{\prime}, j^{\prime}\right)$

$(7) \Longleftrightarrow \alpha \vdash \& l e \sim_{r}$ \&la

(8) $\Longrightarrow \alpha \vdash \& l e \sim_{e} \& l a$

Definition

Definition $3 .(6)$

Definition 4 , (7)

7. For $(\tau) e, \operatorname{Ex} \operatorname{Tr}((\tau) e)=(\tau) r a$, where $r a=\operatorname{Ex} \operatorname{Tr}(e), e, r a,(\tau) e$ and $(\tau) r a$ are all right-value expressions.
(1) $\quad \alpha \vdash e \sim_{e} r a$
(2) $\quad \alpha \vdash M \sim s$
hypothesis
(3) $\quad(G, E \vdash(\tau) e, M \Rightarrow v) \wedge((\tau) r a, \sigma, s,|\sigma|+1) \Downarrow n$ given condition
$\left(G, E \vdash e, M \Rightarrow v_{1}\right) \wedge v=(\tau) v_{1} \wedge\left((r a, \sigma, s,|\sigma|+1) \| n_{1}\right) \wedge n_{n}=(\tau) n_{1}$
(5) $\Longrightarrow \alpha \vdash v_{1} \sim n_{1} \wedge v=(\tau) v_{1} \wedge n=(\tau) n_{1} \quad$ Definition 2. 31. 34. R4, (3)
(6) $\Longrightarrow \quad v_{1}=n_{1} \wedge v=(\tau) v_{1} \wedge n=(\tau) n_{1}$
(7) $\Longrightarrow \quad v=n$
$v_{1}$ and $n_{1}$ are both non-pointer values, (5)
(8) $\Longrightarrow \alpha \vdash v \sim n$
definition of $\alpha \vdash v \sim n$, (7)
$(9) \Longleftrightarrow \alpha \vdash(\tau) e \sim_{r}(\tau) r a$
Definition 3 (8)
$(10) \Longrightarrow \alpha \vdash(\tau) e \sim_{e}(\tau) r a$
Definition 4 (9)

In a similar way, $\alpha \vdash o p_{1} e_{1} \sim_{e} o p_{1} \operatorname{ExTr}\left(e_{1}\right)$ can be proved.

8. For $e_{1}$ aop $e_{2}, \operatorname{ExTr}\left(e_{1}\right.$ aop $\left.e_{2}\right)=r a_{1}$ aop $r a_{2}$, where $r a_{1}=\operatorname{Ex} \operatorname{Tr}\left(e_{1}\right)$, $r a_{2}=\operatorname{Ex} \operatorname{Tr}\left(e_{2}\right)$ and all expressions are right-value expressions. To prove the conclusion, the following three cases need to be taken into account. 
Case 1: $e_{1}, e_{2}, r a_{1}$ and $r a_{2}$ are of non-pointer type.

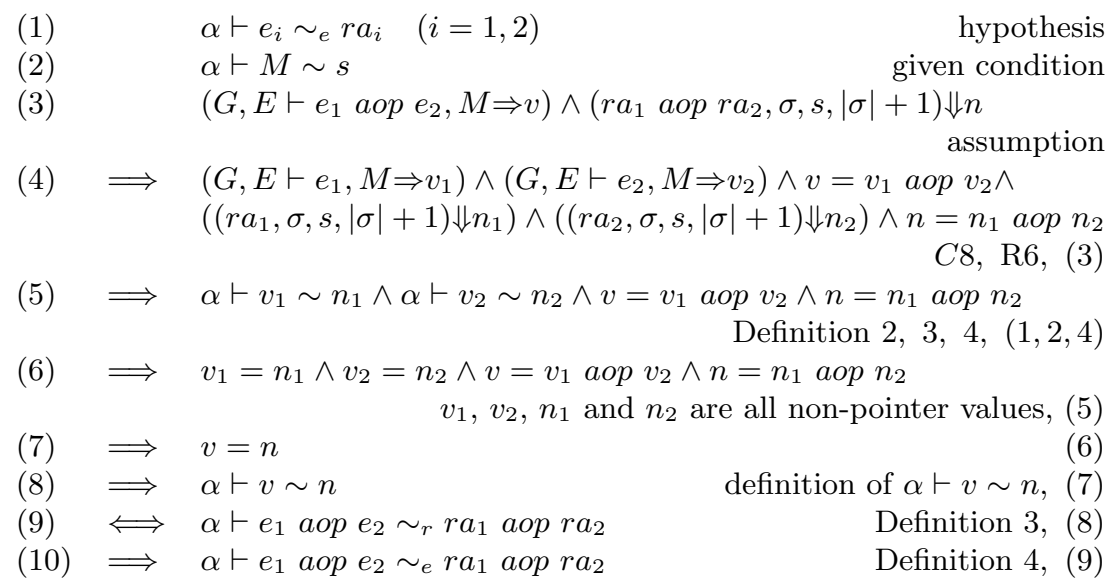

Case 2: both $e_{1}$ and $r a_{1}$ are of pointer type $\tau *$ while both $e_{2}$ and $r a_{2}$ are of integer type. Here, aop $=+\mid-$.

$$
\begin{aligned}
& \quad \alpha \vdash e_{i} \sim_{e} r a_{i} \quad(i=1,2) \quad \text { hypothesis } \\
& \text { (2) } \alpha \vdash M \sim s \quad \text { given condition } \\
& \text { (3) } G, E \vdash e_{1} \text { aop } e_{2}, M \Rightarrow p \operatorname{tr}(b, j) \quad \text { assumption }
\end{aligned}
$$

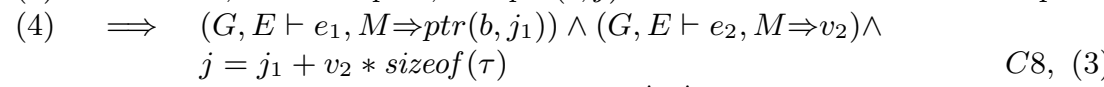

$$
\begin{aligned}
& \text { (5) } \quad\left(r a_{1} \text { aop } r a_{2}, \sigma, s,|\sigma|+1\right) \Downarrow p \operatorname{tr}\left(b^{\prime}, j^{\prime}\right) \quad \text { assumption } \\
& (6) \quad \Longrightarrow \quad\left(r a_{1}, \sigma, s,|\sigma|+1\right) \Downarrow p \operatorname{tr}\left(b^{\prime}, j_{1}^{\prime}\right) \wedge\left(r a_{2}, \sigma, s,|\sigma|+1\right) \Downarrow n_{2} \wedge \\
& j^{\prime}=j_{1}^{\prime}+n_{2} * \operatorname{sizeof}(\tau) \\
& \text { (7) } \quad\left(G, E \vdash e_{1}, M \Rightarrow p \operatorname{tr}\left(b, j_{1}\right)\right) \wedge\left(G, E \vdash e_{2}, M \Rightarrow v_{2}\right) \wedge \\
& \left(r a_{1}, \sigma, s,|\sigma|+1\right) \Downarrow p \operatorname{tr}\left(b^{\prime}, j_{1}^{\prime}\right) \wedge\left(r a_{2}, \sigma, s,|\sigma|+1\right) \Downarrow n_{2} \\
& \text { (8) } \Longrightarrow \quad \alpha \vdash \operatorname{ptr}\left(b, j_{1}\right) \sim \operatorname{ptr}\left(b^{\prime}, j_{1}\right) \wedge \alpha \vdash v_{2} \sim n_{2} \\
& (9) \quad \Longrightarrow \quad \alpha \vdash p \operatorname{tr}\left(b, j_{1}\right) \sim p \operatorname{tr}\left(b^{\prime}, j_{1}\right) \wedge v_{2}=n_{2} \\
& \text { Definition 2, 3, 4, }(1,2,7) \\
& v_{2} \text { and } n_{2} \text { are both non-pointer values, (8) } \\
& (10) \quad \Longrightarrow \quad \alpha \vdash \operatorname{ptr}\left(b, j_{1}+v_{2} * \operatorname{sizeof}(\tau)\right) \sim \operatorname{ptr}\left(b, j_{1}^{\prime}+n_{2} * \operatorname{sizeof}(\tau)\right) \\
& (11) \quad \Longrightarrow \quad \alpha \vdash p \operatorname{tr}(b, j) \sim p \operatorname{tr}\left(b^{\prime}, j^{\prime}\right) \\
& \text { Lemma 1, (9) } \\
& (4,6,10) \\
& (12) \Longleftrightarrow \alpha \vdash e_{1} \text { aоp } e_{2} \sim_{r} r a_{1} \text { aop } r a_{2} \quad \text { Definition 3. (11) } \\
& (13) \Longrightarrow \alpha \vdash e_{1} \text { aop } e_{2} \sim_{e} r a_{1} \text { aop ra } \quad \text { Definition (12) }
\end{aligned}
$$

Case 3: both $e_{2}$ and $r a_{2}$ are of pointer type $\tau *$ while both $e_{1}$ and $r a_{1}$ are of integer type. The proof is similar to Case 2.

For $e_{1}$ rop $e_{2}, e_{1} \& \& e_{2}$ and $e_{1} \| e_{2}$, similar proofs can be given.

9. For $e_{1} ? e_{2}: e_{3}, \operatorname{ExTr}\left(e_{1} ? e_{2}: e_{3}\right)=i f(b)$ then $r a_{2}$ else $r a_{3}$, where $b=$ $\operatorname{ExTr}\left(e_{1}\right), r a_{2}=\operatorname{ExTr}\left(e_{2}\right), r a_{3}=\operatorname{Ex} \operatorname{Tr}\left(e_{3}\right)$ and all expressions are right- 
value expressions.

(1) $\quad\left(\alpha \vdash e_{1} \sim_{e} b\right) \wedge\left(\alpha \vdash e_{2} \sim_{e} r a_{2}\right) \wedge\left(\alpha \vdash e_{3} \sim_{e} r a_{3}\right) \quad$ hypothesis

(2) $\quad \alpha \vdash M \sim s \quad$ given condition

(3) $\left(G, E \vdash e_{1} ? e_{2}: e_{3}, M \Rightarrow v\right) \wedge\left(i f(b)\right.$ then $r a_{2}$ else $\left.r a_{3}, \sigma, s,|\sigma|+1\right) \Downarrow n$

$(4) \Longrightarrow\left(G, E \vdash e_{1}, M \Rightarrow v_{1}\right) \wedge\left(v_{1}=\operatorname{true} \wedge\left(G, E \vdash e_{2}, M \Rightarrow v\right) \vee\right.$ $v_{1}=$ false $\left.\wedge\left(G, E \vdash e_{3}, M \Rightarrow v\right)\right) \wedge(b, \sigma, s,|\sigma|+1) \Downarrow t \wedge(t=$ true $\wedge$ $\left(r a_{2}, \sigma, s,|\sigma|+1\right) \Downarrow n \vee t=$ false $\left.\wedge\left(r_{3}, \sigma, s,|\sigma|+1\right) \Downarrow n\right)$

$C 9, C 10, \mathrm{R} 7, \mathrm{R} 8,(3)$

$(5) \Longrightarrow v_{1}=t=$ true $\wedge \alpha \vdash n \sim v \vee v_{1}=t=$ false $\wedge \alpha \vdash n \sim v$

(6) $\Longrightarrow \alpha \vdash v \sim n$

Definition $2,3,4,(1,2,4)$

$(7) \Longleftrightarrow \alpha \vdash e_{1} ? e_{2}: e_{3} \sim_{r}$ if $(b)$ then $r a_{2}$ else $r a_{3}$

(8) $\Longrightarrow \quad \alpha \vdash e_{1} ? e_{2}: e_{3} \sim_{e}$ if $(b)$ then $r a_{2}$ else $r a_{3}$

10. For a function call $x\left(e_{1}, \ldots, e_{k}\right)$, if $x$ points to a user-defined function, $\operatorname{ExTr}\left(x\left(e_{1}, \ldots, e_{k}\right)\right)=\operatorname{ext} f\left(r a_{1}, \ldots, r a_{k}, R V a l\right)$ otherwise $\operatorname{ExTr}\left(x\left(e_{1}, \ldots\right.\right.$, $\left.\left.e_{k}\right)\right)=\operatorname{ext} f\left(r a_{1}, \ldots, r a_{k}\right)$, where $r a_{i}=\operatorname{ExTr}\left(e_{i}\right)$ for $1 \leq i \leq k, f=$ $\operatorname{Ex} \operatorname{Tr}(x)$ and all expressions are right-value expressions. The following two cases need to be considered.

Case 1: $x\left(e_{1}, \ldots, e_{k}\right)$ is a call of an external function extern $\tau i d(p a r)$, where $\operatorname{par}=\left(\tau_{1} y_{1}, \ldots, \tau_{k} y_{k}\right)$. By induction hypothesis, $f$ is also a call of extern $\tau$ id (par) but in the form ext $f\left(r a_{1}, \ldots, r a_{k}\right)$.

$$
\begin{array}{lll}
(1) & & \alpha \vdash e_{i} \sim_{e} r a_{i} \quad(i=1, \ldots, k) \\
(2) & & \alpha \vdash M \sim s \\
(3) & & G, E \vdash x\left(e_{1}, \ldots, e_{k}\right), M \Rightarrow v \\
(4) \quad \Longrightarrow \quad & G, E \vdash x\left(e_{1}, \ldots, e_{k}\right), M \stackrel{+}{\Rightarrow} v, M
\end{array}
$$

hypothesis given condition

$(5) \Longrightarrow \bigwedge_{i=1}^{k}\left(G, E \vdash e_{i}, M \Rightarrow v_{i}\right) \wedge\left(G \vdash i d\left(v_{1}, \ldots, v_{k}\right), M \stackrel{t}{\Rightarrow} v, M\right)$

(6) $\Longrightarrow \quad \bigwedge_{i=1}^{k}\left(G, E \vdash e_{i}, M \Rightarrow v_{i}\right) \wedge v=i d\left(v_{1}, \ldots, v_{k}\right)$

(7) $\quad\left(\right.$ ext $\left.f\left(r a_{1}, \ldots, r a_{k}\right), \sigma, s,|\sigma|+1\right) \Downarrow n$

(8) $\Longrightarrow \quad \bigwedge_{i=1}^{k}\left(r a_{i}, s, \sigma,|\sigma|+1\right) \Downarrow n_{i} \wedge n=i d\left(n_{1}, \ldots, n_{k}\right)$ assumption

$$
\bigwedge_{i=1}^{k}\left(G, E \vdash e_{i}, M \Rightarrow v_{i}\right) \wedge v=i d\left(v_{1}, \ldots, v_{k}\right) \wedge
$$$$
\bigwedge_{i=1}^{k}\left(r a_{i}, s, \sigma,|\sigma|+1\right) \Downarrow n_{i} \wedge n=i d\left(n_{1}, \ldots, n_{k}\right)
$$

(10) $\Longrightarrow \quad \bigwedge_{i=1}^{k} \alpha \vdash v_{i} \sim n_{i} \wedge v=i d\left(v_{1}, \ldots, v_{k}\right) \wedge n=i d\left(n_{1}, \ldots, n_{k}\right)$

(11) $\Longrightarrow \alpha \vdash v \sim n$

Definition 2, 3, $4,(1,2,9)$

$(12) \Longleftrightarrow \alpha \vdash x\left(e_{1}, \ldots, e_{k}\right) \sim_{r} \operatorname{ext} f\left(r a_{1}, \ldots, r a_{k}\right)$

$(13) \Longrightarrow \alpha \vdash x\left(e_{1}, \ldots, e_{k}\right) \sim_{e}$ ext $f\left(r a_{1}, \ldots, r a_{k}\right)$

Case 2: $x$ points to a user-defined function $\tau i d($ par $)\{d c l ; c s\}$, where par $=$ $\left(\tau_{1} y_{1}, \ldots, \tau_{k} y_{k}\right)$ and $d c l ; c s$ is the body of the function. By induction hypothesis, $f$ points to function $i d\left(\tau_{1} y_{1}, \ldots, \tau_{k} y_{k}, \tau R V a l\right)\{\phi\}$, where $\phi$ 
is translated from $d c l ; c s(\phi=S t m t T r(d c l ; c s)$ see Algorithm 4.6).

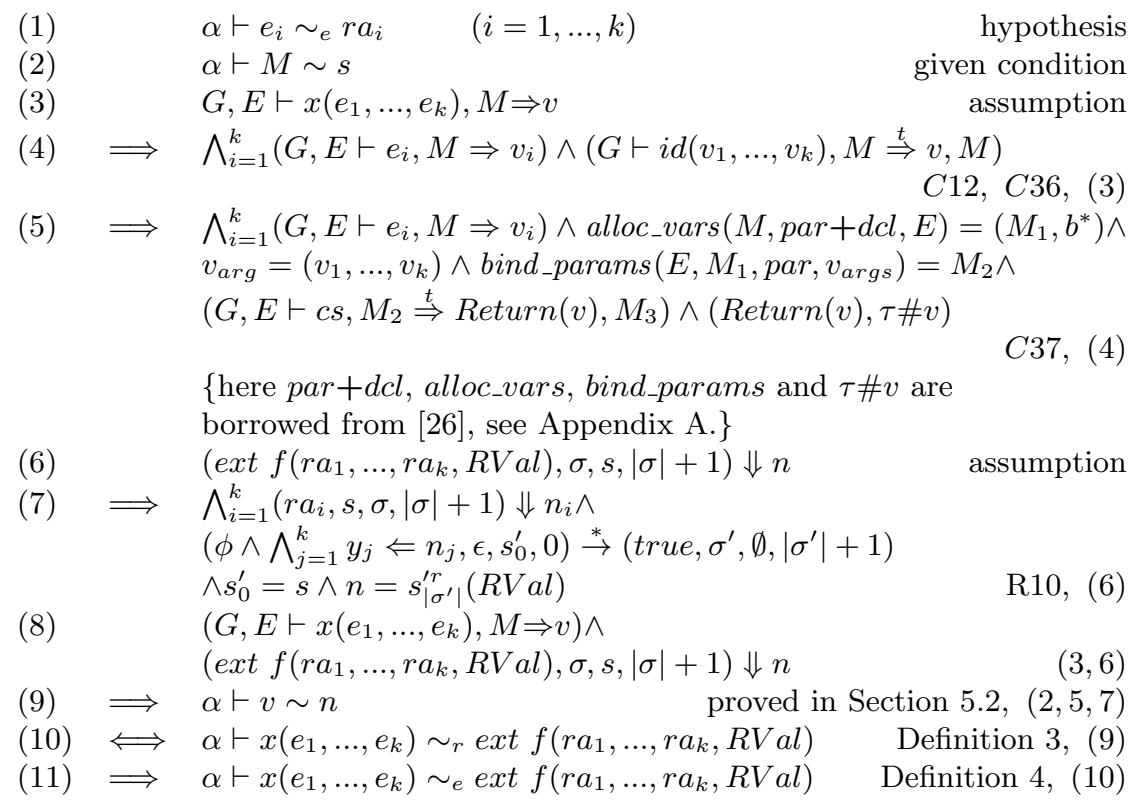

\subsection{Proof of statement equivalence}

Theorem 2. Suppose an Xd-C statement cs is transformed to an MSVL statement $m s$ by Algorithm 4.6 along with transforming an $X d-C$ program to an $M S V L$ program. That is, ms $=\operatorname{Stmt} \operatorname{Tr}(c s)$. For a given $\alpha$, any $M \in \mathbb{M}$ and $s_{i} \in \mathbb{S}$, if $\alpha \vdash M \sim s_{i}$, then $\alpha \vdash c s \sim_{s} m s$.

Proof: The proof proceeds by induction on the rules of operational semantics of statements. To do so, we consider two cases: terminating and diverging statement equivalences. That is,

(1) Terminating case:

$$
G, E \vdash c s, M \stackrel{t}{\Rightarrow}\left(\text { out }, M^{\prime}\right) \Longrightarrow P\left(c s, M, M^{\prime}, \text { out }\right)
$$

where

$$
\begin{array}{rlrl}
P\left(c s, M, M^{\prime}, \text { out }\right) \Longleftrightarrow & \left(\alpha \vdash M \sim s_{i} \Longrightarrow\right. \\
& \left(m s, \sigma_{i-1}, s_{i}, i\right) \stackrel{*}{\rightarrow}(\text { true }, \sigma, \emptyset,|\sigma|+1) \wedge \quad \\
& \left.\alpha \vdash M^{\prime} \sim s_{|\sigma|}\right) & \\
& & \text { TER }
\end{array}
$$

(2) Diverging case:

$$
G, E \vdash c s, M \stackrel{T}{\Rightarrow} \infty \Longrightarrow P^{\prime}(c s, M)
$$


where

$$
\begin{aligned}
P^{\prime}(c s, M) \Longleftrightarrow & \left(\alpha \vdash M \sim s_{i} \Longrightarrow(c s, M) \cong\left(c s_{1} ; c s_{2} ; \ldots, M\right) \wedge\right. \\
& \left(m s, \sigma_{i-1}, s_{i}, i\right) \longmapsto\left(m s_{1} ; m s_{2} ; \ldots, \sigma_{i-1}, s_{i}, i\right) \\
& \left.\wedge \bigwedge_{j=1}^{\infty}\left(\alpha \vdash c s_{j} \sim_{t} m s_{j}\right)\right)
\end{aligned}
$$

Base: 1:

1. For rule $T 1$ w.r.t. a null statement ';', the conclusion is trivially true.

2. For rule T2 w.r.t. "break;", $\operatorname{StmtTr}($ break; ) = "break $:=1 "$.

$$
\begin{gathered}
(3) \Longrightarrow \quad \forall y \in \operatorname{Dom}\left(s_{i+1}\right) \backslash\{\text { break, continue, return, } R \text { Val }\} \\
s_{i+1}^{r}(y)=s_{i}^{r}(y) \wedge s_{i+1}^{l}(y)=s_{i}^{l}(y)
\end{gathered}
$$$$
\text { (break } \left.:=1, \sigma_{i-1}, s_{i}, i\right)
$$$$
\longmapsto\left(\bigcirc(\text { break } \Leftarrow 1 \wedge \text { empty }), \sigma_{i-1}, s_{i}, i\right)
$$$$
\rightarrow\left(\text { break } \Leftarrow 1 \wedge \text { empty, } \sigma_{i}, s_{i+1}, i+1\right)
$$$$
\longmapsto\left(\text { empty, } \sigma_{i},\left(s_{i+1}^{l}, s_{i+1}^{r}[1 / \text { break }]\right), i+1\right)
$$$$
\left.\rightarrow \text { (true, } \sigma_{i+1}, \emptyset, i+2\right)
$$

MIN1

$\forall y \in \operatorname{Dom}\left(s_{i}\right) \backslash\{$ break, continue, return, $R$ Val $\}$

$$
\text { (empty, } \sigma_{i}, s_{i+1}, i+1 \text { ) }
$$

(5) $\Longrightarrow \alpha \vdash M \sim s_{i+1}$

(6) $\Longleftrightarrow P($ break; $, M, M$, Break)

given condition Definition 1, $(3,4)$

TER, $(1,5)$

Note that, $s_{i} \neq s_{i+1}$ in the above proof even so $\alpha \vdash M \sim s_{i}$ and $\alpha \vdash M \sim$ $s_{i+1}$ under the consideration without variables break, continue, return and $R V a l$.

In the same way, we can prove that the conclusions are all true for rules $T 3$, T4 and T5 w.r.t. "continue;", "return;" and "return $e$;", respectively.

3. For rule $T 6$ w.r.t. "le $=e ; ", \operatorname{StmtTr}(l e=e ;)=" l a:=r a "$, where $l a=$ 
$\operatorname{ExTr}(l e)$ and $r a=\operatorname{ExTr}(e)$.

$$
\begin{array}{ll}
(1) & \alpha \vdash l e \sim_{e} l a \\
(2) & \alpha \vdash e \sim_{e} r a \\
(3) & \alpha \vdash M \sim s_{i} \\
(4) & (G, E \vdash l e, M \stackrel{l}{\Rightarrow}(b, j)) \wedge\left(l a, \sigma_{i-1}, s_{i}, i\right) \stackrel{l}{\Rightarrow}\left(b^{\prime}, j^{\prime}\right) \\
(5) \quad \Longrightarrow \quad & \alpha \vdash \operatorname{tr}(b, j) \sim \operatorname{tr}\left(b^{\prime}, j^{\prime}\right) \\
(6) & (G, E \vdash e, M \Rightarrow v) \wedge\left(r a, \sigma_{i-1}, s_{i}, i\right) \Downarrow n
\end{array}
$$

(7) $\Longrightarrow \alpha \vdash v \sim n$

assumption

In the MSVL program, we have
(8) $\quad s_{i}^{l}\left(x_{m}\right)=\left(b^{\prime}, j^{\prime}\right)$
(9) $\quad\left(l a:=r a, \sigma_{i-1}, s_{i}, i\right)$

$$
\longmapsto\left(\bigcirc\left(x_{m} \Leftarrow n \wedge \text { empty }\right), \sigma_{i-1}, s_{i}, i\right) \quad \text { UASS, (8) }
$$$$
\rightarrow\left(\left(x_{m} \Leftarrow n \wedge \text { empty }\right), \sigma_{i}, s_{i+1}, i+1\right)
$$
TR1$$
\left.\longmapsto \text { (empty, } \sigma_{i},\left(s_{i+1}^{l}, s_{i+1}^{r}\left[n / x_{m}\right]\right), i+1\right) \quad \text { MIN1 }
$$$$
\text { (10) } \Longrightarrow \quad s_{i+1}^{l}\left(x_{m}\right)=\left(b^{\prime}, j^{\prime}\right) \wedge s_{i+1}^{r}\left(x_{m}\right)=n
$$

Whereas in the Xd-C program, we have

$(11) \quad\left(\alpha \vdash M \sim s_{i}\right) \wedge s_{i}^{l}\left(x_{m}\right)=\left(b^{\prime}, j^{\prime}\right) \wedge\left(\alpha \vdash \operatorname{ptr}(b, j) \sim \operatorname{ptr}\left(b^{\prime}, j^{\prime}\right)\right)$

$(12) \quad \Longrightarrow \quad G, E \vdash x_{c}, M \stackrel{l}{\Rightarrow}(b, j) \quad$ Definition 1 $\alpha$ is an injective function

(13) storeval (type $(l e), M,(b, j), v)=M^{\prime} \square$ assumption

$(14) \quad \Longrightarrow \quad\left(G, E \vdash x_{c}, M^{\prime} \stackrel{l}{\Rightarrow}(b, j)\right) \wedge$ loadval $\left(\operatorname{type}\left(x_{c}\right), M^{\prime},(b, j)\right)=v$

\{here storeval and loadval are borrowed from [26, see Appendix A.\}

$(15) \Longrightarrow\left(G, E \vdash x_{c}, M^{\prime} \stackrel{l}{\Rightarrow}(b, j)\right) \wedge\left(G, E \vdash x_{c}, M^{\prime} \Rightarrow v\right)$

For other variables in the Xd-C and MSVL programs, their locations and variables are not changed. Thus,

$(16) \quad \Longrightarrow \quad\left(l a:=r a, \sigma_{i-1}, s_{i}, i\right) \stackrel{*}{\rightarrow}\left(\right.$ true $\left., \sigma_{i+1}, \emptyset, i+2\right)$

TR2, (9)

$(17) \Longrightarrow \alpha \vdash M^{\prime} \sim s_{i+1}$

$(18) \Longleftrightarrow P\left(l e=e ;, M, M^{\prime}\right.$, Normal $)$

TER, $(16,17)$

Note that, (16) tells us the MSVL program eventually terminates and (17) indicates that final states $M^{\prime}$ and $s_{i+1}$ are equivalent.

Induction:

4. For rule $T 7$ w.r.t. " $c s_{1} ; c s_{2}$ ", $\operatorname{StmtTr}\left(c s_{1} ; c s_{2}\right)=$ " $m s_{1} ; m s_{2}$ ", if there is no break, return or continue in $c s_{1}$, where $m s_{1}=\operatorname{Stmt} \operatorname{Tr}\left(c s_{1}\right)$ and $m s_{2}=\operatorname{StmtTr}\left(c s_{2}\right)$.

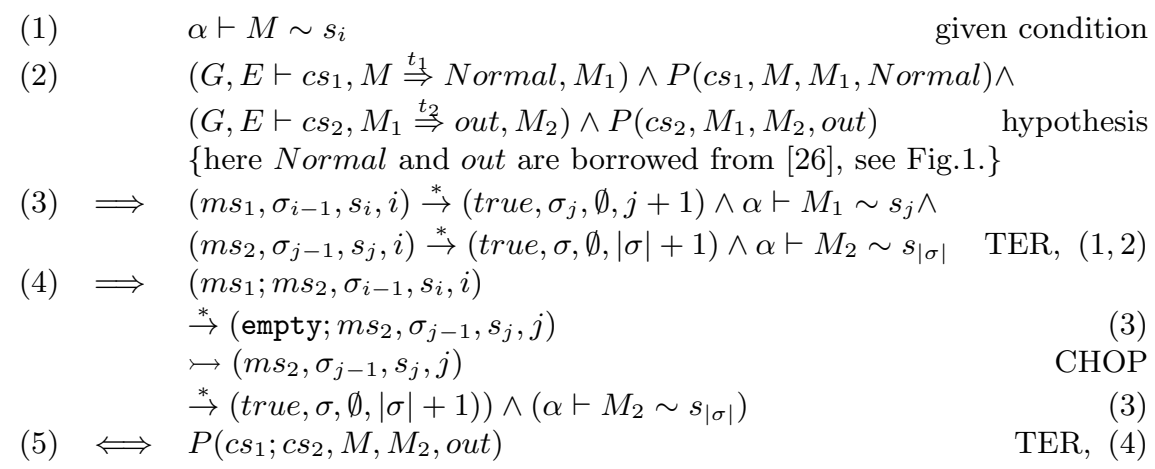


For this rule, if there are break, return and continue in $c s_{1}$, the conclusions can be proved in a similar way.

5. For rule $T 8$ w.r.t. " $c s_{1} ; c s_{2}$ ", $\operatorname{Stmt} \operatorname{Tr}\left(c s_{1} ; c s_{2}\right)=$ " $m s_{1}$; if $($ break $=0)$ then $\left\{m s_{2}\right\}$ else $\left\{\right.$ empty\}", if there is a "break;" statement in $c s_{1}$, where $m s_{1}=\operatorname{Stmt} \operatorname{Tr}\left(c s_{1}\right)$ and $m s_{2}=\operatorname{Stmt} \operatorname{Tr}\left(c s_{2}\right)$.

$$
\begin{aligned}
& \text { (1) } \quad \alpha \vdash M \sim s_{i} \\
& \text { Break, } \left.M^{\prime}\right) \wedge P\left(c_{1}, M, M^{\prime}, \text { Break }\right) \quad \text { hypothesis } \\
& (3) \Longleftrightarrow\left(m s_{1}, \sigma_{i-1}, s_{i}, i\right) \stackrel{*}{\rightarrow}\left(\text { true }, \sigma_{j}, \emptyset, j+1\right) \wedge \alpha \vdash M^{\prime} \sim s_{j} \quad \text { TER, }(1,2) \\
& \text { (4) } \Longrightarrow \quad\left(\left(m s_{1} ; \text { if }(\text { break }=0) \operatorname{then}\left\{m s_{2}\right\} \text { else }\{\text { empty }\}, \sigma_{i-1}, s_{i}, i\right)\right. \\
& \stackrel{*}{\rightarrow}\left(\wedge\{\text { empty }, \text { break } \Leftarrow 1\} ; \text { if }(\text { break }=0) \text { then }\left\{m s_{2}\right\} \text { else }\{\text { empty }\},\right. \\
& \left.\sigma_{j-1}, s_{j}, j\right) \\
& \longmapsto \text { (empty; if }(\text { break }=0) \text { then }\left\{m s_{2}\right\} \text { else }\{\text { empty }\}, \sigma_{j-1}, s_{j}[1 / \text { break }] \\
& \text { j) } \\
& \longrightarrow\left(\text { if }(\text { break }=0) \text { then }\left\{m s_{2}\right\} \text { else }\{\text { empty }\}, \sigma_{j-1}, s_{j}, j\right) \quad \text { CHOP } \\
& \longmapsto\left(\left(\text { break }=0 \wedge m s_{2}\right) \vee(\neg \text { break }=0 \wedge \text { empty }), \sigma_{j-1}, s_{j}, j\right) \quad \text { IF } \\
& \longmapsto\left(\text { empty }, \sigma_{j-1}, s_{j}, j\right) \\
& \left.\rightarrow\left(\text { true }, \sigma_{j}, \emptyset, j+1\right)\right) \wedge\left(\alpha \vdash M^{\prime} \sim s_{j}\right) \\
& \text { (5) } \Longleftrightarrow P\left(c s_{1} ; c s_{2}, M, M^{\prime}, \text { Break }\right) \\
& \text { B3, B4, F1, T1, F2 }
\end{aligned}
$$

In a similar way, it can be proved that

$$
\begin{aligned}
& \left(G, E \vdash c s_{1}, M \stackrel{l}{\Rightarrow} \text { out }, M^{\prime}\right) \wedge \text { out } \in\{\text { Return }, \operatorname{Return}(v), \text { Continue }\} \wedge \\
& P\left(c s_{1}, M, M^{\prime}, \text { out }\right) \\
\Longrightarrow \quad & P\left(c s_{1} ; c s_{2}, M, M^{\prime}, \text { out }\right)
\end{aligned}
$$

6. For rule $T 9$ w.r.t. "if $(e)\left\{c s_{1}\right\}$ else $\left\{c s_{2}\right\} ", \operatorname{Stmt} \operatorname{Tr}\left(\right.$ if $(e)\left\{c s_{1}\right\}$ else $\left.\left\{c s_{2}\right\}\right)=$ "if $(b) \operatorname{then}\left\{m s_{1}\right\}$ else $\left\{m s_{2}\right\}$ ", where $b=\operatorname{Ex} \operatorname{Tr}(e), m s_{1}=\operatorname{Stmt} \operatorname{Tr}\left(c s_{1}\right)$ and $m s_{2}=\operatorname{Stmt} \operatorname{Tr}\left(c s_{2}\right)$.

$$
\begin{aligned}
& \alpha \vdash e \sim_{e} b \\
& \alpha \vdash M \sim s_{i}
\end{aligned}
$$

$$
(G, E \vdash e, M \Rightarrow \text { true }) \wedge\left(G, E \vdash c s_{1}, M \stackrel{t}{\Rightarrow} \text { out }, M^{\prime}\right) \wedge
$$$$
P\left(c s_{1}, M, M^{\prime} \text {, out }\right)
$$

$\Longleftrightarrow\left(m s_{1}, \sigma_{i-1}, s_{i}, i\right) \stackrel{*}{\rightarrow}($ true $, \sigma, \emptyset,|\sigma|+1) \wedge \alpha \vdash M^{\prime} \sim s_{|\sigma|} \quad$ TER, $(2,3)$

$(5) \Longrightarrow\left(b, \sigma_{i-1}, s_{i}, i\right) \Downarrow$ true Definition $3,4,(1,2,3)$

$(6) \Longrightarrow \quad\left(\left(\operatorname{if}(b) \operatorname{then}\left\{m s_{1}\right\}\right.\right.$ else $\left.\left\{m s_{2}\right\}, \sigma_{i-1}, s_{i}, i\right)$ $\longmapsto\left(\left(b \wedge m s_{1}\right) \vee\left(\neg b \wedge m s_{2}\right), \sigma_{i-1}, s_{i}, i\right)$ $\longmapsto\left(m s_{1}, \sigma_{i-1}, s_{i}, i\right)$

In a similar way, for rule $T 10$ w.r.t. "if $(e)\left\{c s_{1}\right\}$ else $\left\{c s_{2}\right\}$ ", it can be proved that

$$
(G, E \vdash e, M \Rightarrow \text { false }) \wedge\left(G, E \vdash c s_{2}, M \stackrel{t}{\Rightarrow} \text { out }, M^{\prime}\right) \wedge P\left(c s_{2}, M, M^{\prime}, \text { out }\right)
$$
$\Longrightarrow P\left(\operatorname{if}(e)\left\{c s_{1}\right\} \mathrm{else}\left\{c s_{2}\right\}, M, M^{\prime}\right.$, out $)$

7. For rule $T 11$ w.r.t. "while $(e)\{c s\} ", \operatorname{Stmt} \operatorname{Tr}($ while $(e)\{c s\})=$ "while $(b)\{m s\} "$, where $b=\operatorname{Ex} \operatorname{Tr}(e)$ and $m s=\operatorname{Stmt} \operatorname{Tr}(c s)$, if there is no break, return 
or continue in $c s$.

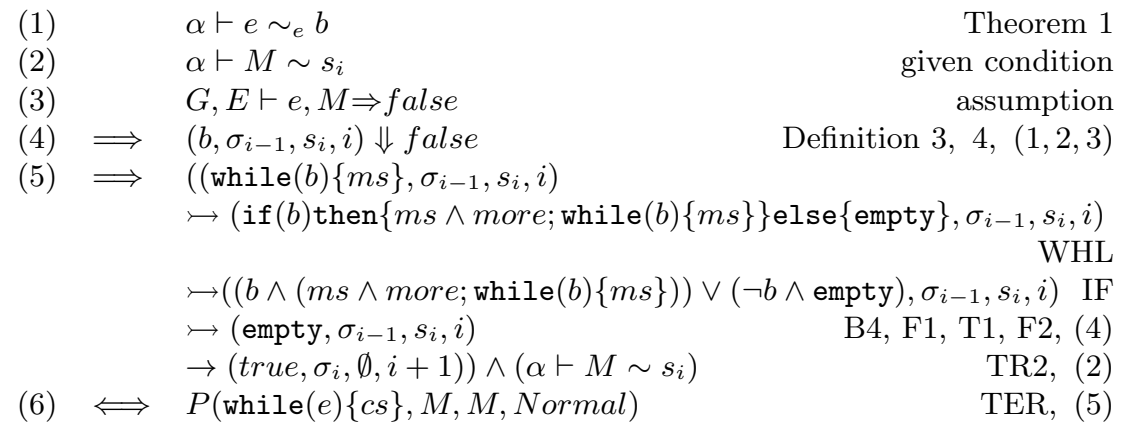

If there are break, return and continue in $c s_{1}$, the conclusions can similarly be proved.

8. For rule $T 12$ w.r.t. "while $(e)\{c s\} ", \operatorname{Stmt} T r($ while $(e)\{c s\})=$ "while $(b$ and break $=0)\{m s\}$; break $:=0 "$, where $b=\operatorname{ExTr}(e)$ and $m s=\operatorname{StmtTr}(c s)$, if there is a "break;" statement in cs. Note that, the initial value of break is 0 .

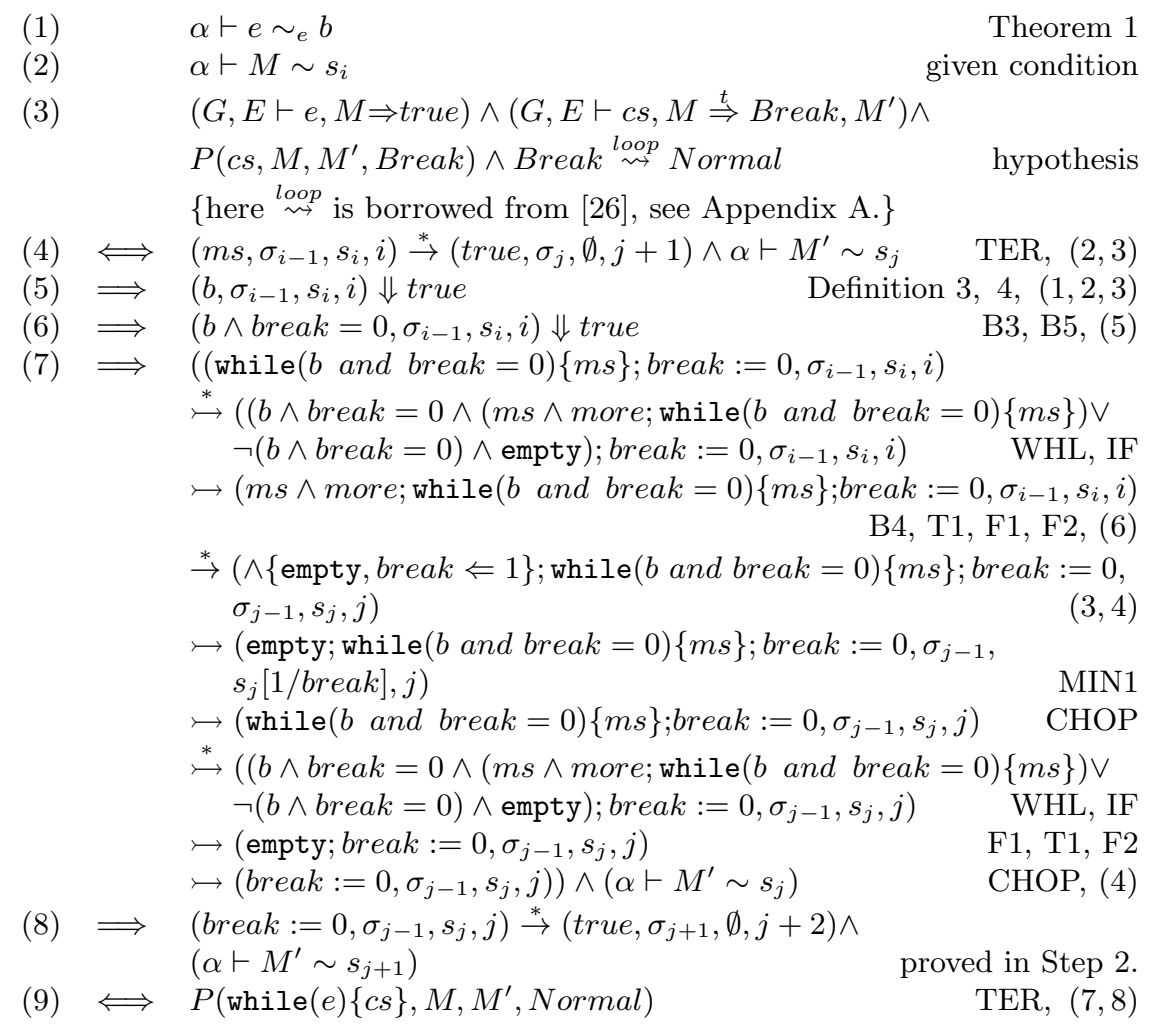


In a similar way, it can be proved that

$$
\begin{aligned}
& (G, E \vdash e, M \Rightarrow \text { true }) \wedge\left(G, E \vdash c s, M \stackrel{t}{\Rightarrow} \text { out }, M^{\prime}\right) \wedge \\
& \text { out } \in\{\text { Return, Return }(v)\} \wedge P\left(\text { cs }, M, M^{\prime}, \text { out }\right) \wedge \text { out } \stackrel{\text { loop }}{\rightsquigarrow} \text { out }{ }^{\prime} \\
\Longrightarrow \quad & P\left(\text { while }(e)\{c s\}, M, M^{\prime}, \text { out }^{\prime}\right)
\end{aligned}
$$

9. For rule $T 13$ w.r.t. "while $(e)\{c s\} ", \operatorname{Stmt} \operatorname{Tr}($ while $(e)\{c s\})=$ "while $(b)\{m s\}$ ", where $b=\operatorname{Ex} \operatorname{Tr}(e)$ and $m s=\operatorname{Stmt} \operatorname{Tr}(c s)$, if there is no break, return or continue in $c s$.

$$
\begin{aligned}
& \alpha \vdash e \sim_{e} b \\
& \alpha \vdash M \sim s_{i} \\
& \text { Theorem } 1 \\
& (G, E \vdash e, M \Rightarrow \text { true }) \wedge\left(G, E \vdash c s, M \stackrel{t_{1}}{\Rightarrow} \text { Normal, } M_{1}\right) \wedge \\
& P\left(c s, M, M_{1}, N o r m a l\right) \wedge\left(G, E \vdash \text { while }(e)\{c s\}, M_{1} \stackrel{t_{2}}{\Rightarrow} \text { out }, M_{2}\right) \wedge \\
& P\left(\text { while }(e)\{c s\}, M_{1}, M_{2}, \text { out }\right) \\
& \text { hypothesis } \\
& (4) \Longleftrightarrow\left(\left(m s, \sigma_{i-1}, s_{i}, i\right) \stackrel{*}{\rightarrow}\left(\text { true }, \sigma_{j}, \emptyset, j+1\right) \wedge \alpha \vdash M_{1} \sim s_{j}\right) \wedge \\
& \left(\text { while }(b)\{m s\}, \sigma_{j-1}, s_{j}, j\right) \stackrel{*}{\rightarrow}(\text { true }, \sigma, \emptyset,|\sigma|+1) \wedge \\
& \left.\alpha \vdash M_{2} \sim s_{|\sigma|}\right) \\
& (5) \quad \Longrightarrow \quad\left(b, \sigma_{i-1}, s_{i}, i\right) \Downarrow \text { true } \\
& (6) \quad \Longrightarrow \quad\left(\left(\text { while }(b)\{m s\}, \sigma_{i-1}, s_{i}, i\right)\right. \\
& \stackrel{*}{\longmapsto}\left((b \wedge(m s \wedge \text { more } ; \text { while }(b)\{m s\})) \vee(\neg b \wedge \text { empty }), \sigma_{i-1}, s_{i}, i\right) \\
& \text { WHL, IF } \\
& \longmapsto\left(m s \wedge \text { more } ; \text { while }(b)\{m s\}, \sigma_{i-1}, s_{i}, i\right) \\
& \text { B4, T1, F1, F2, (5) } \\
& \stackrel{*}{\rightarrow}\left(\text { empty; while }(b)\{m s\}, \sigma_{j-1}, s_{j}, j\right) \\
& \longmapsto\left(\operatorname{while}(b)\{m s\}, \sigma_{j-1}, s_{j}, j\right) \\
& \stackrel{*}{\rightarrow}(\text { true }, \sigma, \emptyset,|\sigma|+1)) \wedge\left(\alpha \vdash M_{2} \sim s_{|\sigma|}\right) \\
& (7) \Longleftrightarrow P\left(\text { while }(e)\{c s\}, M, M_{2}, \text { out }\right)
\end{aligned}
$$

In a similar way, it can be proved that

$$
\begin{aligned}
& (G, E \vdash e, M \Rightarrow \text { true }) \wedge\left(G, E \vdash c s, M \stackrel{t_{1}}{\Rightarrow} \text { Continue }, M_{1}\right) \wedge \\
& P\left(c s, M, M_{1}, \text { Continue }\right) \wedge\left(G, E \vdash \text { while }(e)\{c s\}, M_{1} \stackrel{t_{2}}{\Rightarrow} \text { out }, M_{2}\right) \wedge \\
& P\left(\text { while }(e)\{c s\}, M_{1}, M_{2}, \text { out }\right) \\
\Longrightarrow & P\left(\text { while }(e)\{c s\}, M, M_{2}, \text { out }\right)
\end{aligned}
$$

10. For rules $T 24$ and $T 25$ w.r.t. a function call " $x\left(e_{1}, \ldots, e_{m}\right)$;", $\operatorname{Stmt} \operatorname{Tr}\left(x\left(e_{1}, \ldots\right.\right.$, $\left.\left.e_{m}\right) ;\right)=f\left(r a_{1}, \ldots, r a_{m}, R V a l\right)$, where $f=\operatorname{ExTr}(x)$ and $r a_{k}=\operatorname{Ex} \operatorname{Tr}\left(e_{k}\right)$ for all $1 \leq k \leq m$, if $x$ points to a user-defined function with a return value. We assume $x$ points to $\tau i d($ par $)\{d c l ; c s\}$, where $\operatorname{par}=\left(\tau_{1} y_{1}, \ldots, \tau_{m} y_{m}\right)$. Thus, $f$ points to function $i d\left(\tau_{1} y_{1}, \ldots, \tau_{m} y_{m}, \tau R V a l\right)\{m d c l ; m s\}$ trans- 
lated from $\tau i d(p a r)\{d c l ; c s\}$, where $m d c l=\operatorname{DecTr}(d c l)$ and $m s=\operatorname{Stmt} \operatorname{Tr}(c s)$.

$$
\begin{aligned}
& \alpha \vdash e_{k} \sim_{e} r a_{k} \quad(1 \leq k \leq m) \\
& \alpha \vdash M \sim s_{i} \\
& \bigwedge_{k=1}^{m}\left(G, E \vdash e_{k}, M \Rightarrow v_{k}\right) \wedge v_{\text {args }}=\left(v_{1}, \ldots, v_{m}\right) \wedge \\
& \text { alloc_vars }(M, p a r+d c l, E)=\left(M_{1}, b^{*}\right) \wedge \\
& \text { bind_params }\left(E, M_{1}, \text { par, } v_{\text {args }}\right)=M_{2} \wedge \\
& \left(G, E \vdash c s, M_{2} \stackrel{t}{\Rightarrow} \operatorname{Return}(v), M_{3}\right) \wedge(\operatorname{Return}(v), \tau \# v) \wedge \\
& M_{4}=\operatorname{free}\left(M_{3}, b^{*}\right) \wedge P\left(c s, M_{2}, M_{3}, \operatorname{Return}(v)\right) \quad \text { hypothesis } \\
& \{\text { here alloc_vars, bind_params, Return }(v) \text { and free are borrowed } \\
& \text { from [26], see Appendix A.\} } \\
& \alpha \vdash M_{2} \sim s_{t} \\
& \text { assumption } \\
& (5) \Longrightarrow\left(m s, \sigma_{t-1}, s_{t}, t\right) \stackrel{*}{\rightarrow}\left(\text { true }, \sigma_{j}, \emptyset, j+1\right) \wedge\left(\alpha \vdash M_{3} \sim s_{j}\right) \quad \text { TER, }(3,4) \\
& \left(f\left(r a_{1}, \ldots, r a_{m}, R V a l\right), \sigma_{i-1}, s_{i}, i\right) \\
& \longmapsto\left(i d\left(r a_{1}, \ldots, r a_{m}, R V a l\right), \sigma_{i-1}, s_{i}, i\right) \\
& \longmapsto\left(\left(\wedge_{k=1}^{m} \tau_{k} y_{k} \Leftarrow r a_{k} \wedge m d c l\right) ; m s ; \bigcirc\left(\text { ext } m f r e e\left(y_{1}, \ldots, y_{m}, m d c l\right)\right.\right. \\
& \left.\wedge \text { empty), } \sigma_{i-1}, s_{i}, i\right) \\
& \text { \{here } m \text { free is defined in Section } 3.2 .5 \text { \} } \\
& \stackrel{*}{\rightarrow}\left(m s ; \bigcirc\left(\text { ext } m f r e e\left(y_{1}, \ldots, y_{m}, m d c l\right) \wedge \text { empty }\right), \sigma_{t-1}, s_{t}, t\right)
\end{aligned}
$$

In the Xd-C program, the memory state transfers from $M$ to $M_{2}$. Compared to $M, M_{2}$ allocates memory blocks to variables $y_{1}, \ldots, y_{m}$ and variables in $d c l$, and assigns $v_{k}$ to $y_{k}$ for $1 \leq k \leq m$. Whereas in the MSVL program, the state transfers from $s_{i}$ to $s_{t}$. We assume $\left(r a_{k}, \sigma_{i-1}, s_{i}, i\right) \Downarrow n_{k}$ for all $1 \leq k \leq m$. Thus, $\alpha \vdash v_{k} \sim n_{k}$ due to (1) and (2). Compared to $s_{i}, s_{t}$ also allocates memory blocks to variables $y_{1}, \ldots, y_{m}$ and variables in $m d c l$, and assigns $n_{k}$ to $y_{k}$ for $1 \leq k \leq m$. Hence, $\alpha \vdash M_{2} \sim s_{t}$. Since $\left(G, E \vdash c s, M_{2} \stackrel{t}{\Rightarrow} \operatorname{Return}(v), M_{3}\right)$, "return $e$;" must be executed at the last state of executing $c s$, and $\left(G, E \vdash e, M_{3} \Rightarrow v\right)$. Thus, $R V a l \Leftarrow r a$ must be executed at the last state of executing $m s$, where $r a=\operatorname{ExTr}(e)$. We assume $\left(r a, \sigma_{j-1}, s_{j}, j\right) \Downarrow n$. Accordingly, the reduction continues as follows:

$$
\begin{aligned}
& \left(m s ; \bigcirc\left(\text { ext } m f r e e\left(y_{1}, \ldots, y_{m}, m d c l\right) \wedge \text { empty }\right), \sigma_{t-1}, s_{t}, t\right) \\
& \stackrel{*}{\rightarrow}\left(R V a l \Leftarrow r a \wedge \text { empty; } \bigcirc\left(\text { ext } m f r e e\left(y_{1}, \ldots, y_{m}, m d c l\right) \wedge \text { empty }\right)\right. \text {, } \\
& \left.\sigma_{j-1}, s_{j}, j\right) \\
& \longmapsto\left(\text { empty; } \bigcirc\left(\text { ext } m f r e e\left(y_{1}, \ldots, y_{m}, m d c l\right) \wedge \text { empty }\right), \sigma_{j-1}\right. \text {, } \\
& \left.\left(s_{j}^{l}, s_{j}^{r}[n / R V a l]\right), j\right) \\
& \rightarrow\left(\text { ext } m f r e e\left(y_{1}, \ldots, y_{m}, m d c l\right) \wedge \text { empty }, \sigma_{j}, s_{j+1}, j+1\right) \\
& \rightarrow\left(\text { true }, \sigma_{j+1}, \emptyset, j+2\right)
\end{aligned}
$$

According to (5), we have $\alpha \vdash M_{3} \sim s_{j}$. Further, $\alpha \vdash v \sim s_{j}^{r}(R V a l)$ due to $\alpha \vdash e \sim_{e} r a$. $M_{4}$ just removes $y_{1}, \ldots, y_{m}$ and variables in $d c l$ from $M_{3}$ while $s_{j+1}$ just removes $y_{1}, \ldots, y_{m}$ and variables in $m d c l$ from $s_{j}$. Thus, the locations and values of variables are not changed and $\alpha \vdash M_{4} \sim s_{j+1}$. Therefore,

$$
\begin{aligned}
(8) \Longrightarrow & \left(f\left(r a_{1}, \ldots, r a_{m}, R V a l\right), \sigma_{i-1}, s_{i}, i\right) \stackrel{*}{\rightarrow}\left(\text { true }, \sigma_{j+1}, \emptyset, j+2\right) \wedge \\
& \alpha \vdash M_{4} \sim s_{j+1} \\
(9) \Longrightarrow & P\left(x\left(e_{1}, \ldots, e_{m}\right) ;, M, M_{4}, \text { out }\right)
\end{aligned}
$$


In a similar way, if $x$ points to a user-defined function without a return value, it can be proved that $P\left(x\left(e_{1}, \ldots, e_{m}\right) ;, M, M_{4}\right.$, out $)$ holds.

11. For rules $T 24$ and $T 26$ w.r.t. a function call " $x\left(e_{1}, \ldots, e_{m}\right) ;$;, $\operatorname{Stmt} \operatorname{Tr}\left(x\left(e_{1}, \ldots\right.\right.$, $\left.\left.e_{m}\right) ;\right)=\operatorname{ext} f\left(r a_{1}, \ldots, r a_{m}\right)$, where $f=\operatorname{ExTr}(x)$ and $r a_{k}=\operatorname{ExTr}\left(e_{k}\right)$ for all $1 \leq k \leq m$ and $x$ points to an external function extern $[\tau \mid$ void $]$ id(par $)$, where $\operatorname{par}=\left(\tau_{1} y_{1}, \ldots, \tau_{m} y_{m}\right)$.

$$
\begin{aligned}
& \text { (1) }\left(\wedge\left\{\bigcirc \text { empty, ext } f\left(r a_{1}, \ldots, r a_{m}\right)\right\}, \sigma_{i-1}, s_{i}, i\right) \\
& \rightarrow\left(\text { empty, } \sigma_{i}, s_{i+1}, i+1\right) \quad \text { EXT2 } \\
& \rightarrow\left(\text { true }, \sigma_{i+1}, \emptyset, i+2\right) \wedge s_{i}=s_{i+1} \quad \text { TR2 } \\
& (2) \quad \Longrightarrow \quad\left(\left(\wedge\left\{\bigcirc \text { empty, ext } f\left(r a_{1}, \ldots, r a_{m}\right)\right\}, \sigma_{i-1}, s_{i}, i\right)\right. \\
& \left.\stackrel{*}{\rightarrow}\left(\text { true }, \sigma_{i+1}, \emptyset, i+2\right) \wedge \alpha \vdash M \sim s_{i+1}\right) \\
& (3) \Longleftrightarrow P\left(x\left(e_{1}, \ldots, e_{m}\right), M, M, \text { out }\right)
\end{aligned}
$$

Note that, (1) indicates that $s_{i}=s_{i+1}$ and $\left\langle s_{i}\right\rangle$ is a model of $\operatorname{ext} f\left(n_{1}, \ldots, n_{m}\right)$.

Similar to Step 6, we can prove that for the rules of switch statements, the conclusions are true, and similar to Steps 7, 8 and 9, we can prove for the rules of for loops, the conclusions are true.

Case 2:

Base:

1. For rule $D 1$ w.r.t. "while $($ e) $\{\mathrm{cs}\} ", \operatorname{Stmt} \operatorname{Tr}($ while $(e)\{c s\})=$ "while $(b)\{m s\} "$ ", where $b=\operatorname{Ex} \operatorname{Tr}(e)$ and $m s=\operatorname{Stmt} \operatorname{Tr}(c s)$, if there is no break, return or continue in $c s$. $G, E \vdash$ while $(e)\{c s\}, M \stackrel{T}{\Rightarrow} \infty$.

$(4) \Longrightarrow \quad(\operatorname{while}(e)\{c s\}, M) \cong(c s ; \operatorname{while}(e)\{c s\}, M) \cong(c s ; c s ; \ldots, M)$

$(5) \quad \Longrightarrow \quad\left(b, \sigma_{i-1}, s_{i}, i\right) \Downarrow$ true

$(6) \quad \Longrightarrow \quad\left(\operatorname{while}(b)\{m s\}, \sigma_{i-1}, s_{i}, i\right)$ $\stackrel{*}{\longmapsto}\left(m s ; \operatorname{while}(b)\{m s\}, \sigma_{i-1}, s_{i}, i\right)$

WHL, IF, (5) $\stackrel{*}{\longmapsto}\left(m s ; m s ; \ldots, \sigma_{i-1}, s_{i}, i\right)$

$(7) \Longrightarrow \alpha \vdash c s \sim_{t} m s$

$$
\alpha \vdash M \sim s_{i} \quad \text { given condition }
$$

$(8) \Longleftrightarrow P^{\prime}\left(c s ; c s^{\prime}, M\right)$

WHL, IF, $(1,2,3)$

Case 1

DV, $(4,6,7)$

If there are break, return and continue in $c s$, the conclusions can similarly be proved.

Induction:

2. For rule $D 2$ w.r.t. "cs; $c s^{\prime \prime}$, $\operatorname{Stmt} \operatorname{Tr}\left(c s ; c s^{\prime}\right)=$ " $m s ; m s$ ", where $m s=$ $\operatorname{Stmt} \operatorname{Tr}(c s)$ and $m s^{\prime}=\operatorname{Stmt} \operatorname{Tr}\left(c s^{\prime}\right)$, if there is no break, return or 
continue in $c s .(G, E \vdash c s, M \stackrel{T}{\Rightarrow} \infty)$ and $\left(G, E \vdash c s ; c s^{\prime}, M \stackrel{T}{\Rightarrow} \infty\right)$.

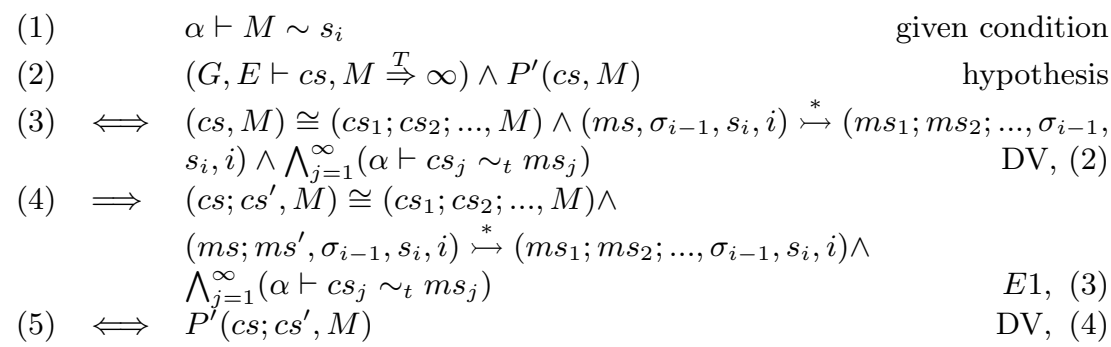

If there are break, return and continue in $c s$, the conclusions can be proved in a similar way.

3. For rule D3 w.r.t. "cs; $c s^{\prime \prime}$, $\operatorname{Stmt} \operatorname{Tr}\left(c s ; c s^{\prime}\right)=$ " $m s ; m s$ ", where $m s=$ $\operatorname{Stmt} \operatorname{Tr}(c s)$ and $m s^{\prime}=\operatorname{Stmt} \operatorname{Tr}\left(c s^{\prime}\right)$, if there is no break, return or continue in cs. $G, E \vdash c s, M \stackrel{t}{\Rightarrow}$ Normal, $M_{1}, G, E \vdash c s^{\prime}, M_{1} \stackrel{T}{\Rightarrow} \infty$ and $G, E \vdash c s ; c s^{\prime}, M_{1} \stackrel{T}{\Rightarrow} \infty$.

$$
\alpha \vdash M \sim s_{i} \quad \text { given condition }
$$

$\left(G, E \vdash c s, M \stackrel{t}{\Rightarrow}\right.$ Normal,$\left.M_{1}\right) \wedge\left(G, E \vdash c s^{\prime}, M_{1} \stackrel{T}{\Rightarrow} \infty\right) \wedge$ $P^{\prime}\left(c s^{\prime}, M_{1}\right)$

(3) $\Longrightarrow P\left(c s, M, M_{1}\right.$, Normal $) \quad$ Terminating Statement Equivalence, (2)

(4) $\Longrightarrow\left(m s, \sigma_{i-1}, s_{i}, i\right) \stackrel{*}{\rightarrow}\left(\right.$ true $\left., \sigma_{t}, \emptyset, t+1\right) \wedge \alpha \vdash M_{1} \sim s_{t} \wedge$ $\left(c s^{\prime}, M_{1}\right) \cong\left(c s_{1} ; c s_{2} ; \ldots, M_{1}\right) \wedge$ $\left(m s^{\prime}, \sigma_{t-1}, s_{t}, t\right) \stackrel{*}{\longmapsto}\left(m s_{1} ; m s_{2} ; \ldots, \sigma_{t-1}, s_{t}, t\right) \wedge$ $\bigwedge_{j=1}^{\infty}\left(\alpha \vdash c s_{j} \sim_{t} m s_{j}\right)$

$(5) \Longrightarrow\left(c s ; c s^{\prime}, M\right) \cong\left(c s ; c s_{1} ; c s_{2} ; \ldots, M\right) \wedge$ $\left(m s ; m s^{\prime}, \sigma_{i-1}, s_{i}, i\right) \stackrel{*}{\longmapsto}\left(m s ; m s_{1} ; m s_{2} ; \ldots, \sigma_{i-1}, s_{i}, i\right) \wedge$ $\bigwedge_{j=1}^{\infty}\left(\alpha \vdash c s_{j} \sim_{t} m s_{j}\right) \wedge \alpha \vdash c s \sim_{t} m s \quad E 2,(3,4)$

(6) $\Longleftrightarrow P^{\prime}\left(c s ; c s^{\prime}, M\right)$

If there are break, return and continue in $c s$, the conclusions are also true.

4. For rule $D 4$ w.r.t. "if $(e)\{c s\}$ else $\left\{c s^{\prime}\right\}$ ", $\operatorname{Stmt} \operatorname{Tr}\left(\right.$ if $(e)\{c s\}$ else $\left.\left\{c s^{\prime}\right\}\right)=$ "if(b)then $\{m s\}$ else $\left\{m s^{\prime}\right\}$ ", where $b=\operatorname{ExTr}(e), m s=\operatorname{Stmt} \operatorname{Tr}(c s)$ and $m s^{\prime}=\operatorname{StmtTr}\left(c s^{\prime}\right) . \quad(G, E \vdash e, M \Rightarrow$ true $),(G, E \vdash c s, M \stackrel{T}{\Rightarrow} \infty)$ and 
$\left(G, E \vdash \operatorname{if}(e)\{c s\} \mathrm{else}\left\{c s^{\prime}\right\}, M \stackrel{T}{\Rightarrow} \infty\right)$.

(1) $\quad \alpha \vdash M \sim s_{i} \quad$ given condition

(2) $\alpha \vdash e \sim_{e} b \quad$ Theorem 1

(3) $\quad(G, E \vdash e, M \Rightarrow$ true $) \wedge(G, E \vdash c s, M \stackrel{T}{\Rightarrow} \infty) \wedge P^{\prime}(c s, M)$

$\begin{aligned}(4) \Longrightarrow \quad & (c s, M) \cong\left(c s_{1} ; c s_{2} ; \ldots, M\right) \wedge \\ & \left(m s, \sigma_{i-1}, s_{i}, i\right) \stackrel{*}{\longmapsto}\left(m s_{1} ; m s_{2} ; \ldots, \sigma_{i-1}, s_{i}, i\right)\end{aligned}$

$\wedge \bigwedge_{j=1}^{\infty}\left(\alpha \vdash c s_{j} \sim_{t} m s_{j}\right) \quad$ DV, (3)

(5) $\Longrightarrow \quad\left(\right.$ if $\left.(e)\{c s\} \operatorname{else}\left\{c s^{\prime}\right\}, M\right) \cong(c s, M) \cong\left(c s_{1} ; c s_{2} ; \ldots, M\right) \quad E 3,(3,4)$

$(6) \Longrightarrow\left(b, \sigma_{i-1}, s_{i}, i\right) \Downarrow$ true $\quad$ Definition 3 4 $(1,2,3)$

$(7) \Longrightarrow \quad\left(\right.$ if $(b)$ then $\left.\{m s\} \operatorname{else}\left\{m s^{\prime}\right\}, \sigma_{i-1}, s_{i}, i\right)$

$\stackrel{*}{\longmapsto}\left(m s, \sigma_{i-1}, s_{i}, i\right) \quad$ IF, (6)

$\stackrel{*}{\longmapsto}\left(m s_{1} ; m s_{2} ; \ldots, \sigma_{i-1}, s_{i}, i\right) \wedge \bigwedge_{j=1}^{\infty}\left(\alpha \vdash c s_{j} \sim_{t} m s_{j}\right)$

$(8) \Longleftrightarrow P^{\prime}\left(\operatorname{if}(e)\{c s\} \operatorname{else}\left\{c s^{\prime}\right\}, M\right)$

DV,$(5,7)$

For rule D5 w.r.t. "if $(e)\{c s\}$ else $\left\{c s^{\prime}\right\}$ " and D6 w.r.t. "while(e) $\{\operatorname{cs}\}$ ", we can similarly prove that

(1) $\quad(G, E \vdash e, M \Rightarrow$ false $) \wedge\left(G, E \vdash c s^{\prime}, M \stackrel{T}{\Rightarrow} \infty\right) \wedge P^{\prime}\left(c s^{\prime}, M\right)$

$\Longrightarrow P^{\prime}\left(\operatorname{if}(e)\{c s\} \operatorname{else}\left\{c s^{\prime}\right\}, M\right)$

(2) $\quad(G, E \vdash e, M \Rightarrow$ true $) \wedge(G, E \vdash c s, M \stackrel{T}{\Rightarrow} \infty) \wedge P^{\prime}(c s, M)$

$\Longrightarrow P^{\prime}($ while $(e)\{c s\}, M)$

5. For rule $D 7$ w.r.t. "while (e) $\{\mathrm{cs}\} ", \operatorname{Stmt} \operatorname{Tr}($ while $(e)\{c s\})=$ "while $(b)\{m s\}$ ", where $b=\operatorname{ExTr}(e)$ and $m s=\operatorname{Stmt} \operatorname{Tr}(c s)$, if there is no break, return or continue in $c s$.

(1) $\quad \alpha \vdash M \sim s_{i}$

(2) $\quad \alpha \vdash e \sim \sim_{e} b$

given condition

(3) $\quad(G, E \vdash e, M \Rightarrow$ true $) \wedge\left(G, E \vdash c s, M \stackrel{t}{\Rightarrow}\right.$ Normal, $\left.M_{1}\right) \wedge$

Theorem 1

$\left(G, E \vdash\right.$ while $\left.(e)\{c s\}, M_{1} \stackrel{T}{\Rightarrow} \infty\right) \wedge P^{\prime}\left(\right.$ while $\left.(e)\{c s\}, M_{1}\right)$ hypothesis

(4) $\Longrightarrow P\left(c s, M, M_{1}\right.$, Normal $) \quad$ Terminating Statement Equivalence, $(1,3)$

(5) $\Longrightarrow\left(m s, \sigma_{i-1}, s_{i}, i\right) \stackrel{*}{\rightarrow}\left(\right.$ true $\left., \sigma_{t}, \emptyset, t+1\right) \wedge \alpha \vdash M_{1} \sim s_{t} \quad$ TER, $(1,4)$

(6) $\Longrightarrow \quad\left(\right.$ while $\left.(e)\{c s\}, M_{1}\right) \cong\left(c s_{1} ; c s_{2} ; \ldots, M_{1}\right) \wedge$

$\left(\right.$ while $\left.(b)\{m s\}, \sigma_{t-1}, s_{t}, t\right) \stackrel{*}{\longmapsto}\left(m s_{1} ; m s_{2} ; \ldots, \sigma_{t-1}, s_{t}, t\right) \wedge$

$\bigwedge_{j=1}^{\infty}\left(\alpha \vdash c s_{j} \sim_{t} m s_{j}\right)$

$\mathrm{DV},(3)$

(7) $\Longrightarrow \quad($ while $(e)\{c s\}, M) \cong\left(c s ; c s_{1} ; c s_{2} ; \ldots, M\right)$

$(8) \Longrightarrow\left(b, \sigma_{i-1}, s_{i}, i\right) \Downarrow$ true

$E 2, E 5,(3,6)$

(9) $\Longrightarrow \quad\left(\right.$ while $\left.(b)\{m s\}, \sigma_{i-1}, s_{i}, i\right)$

$\stackrel{*}{\longmapsto}\left(m s \wedge m o r e ;\right.$ while $\left.(b)\{m s\}, \sigma_{i-1}, s_{i}, i\right)$

Definition $3,4,(1,2,3)$

$\stackrel{*}{\longmapsto}\left(m s ; m s_{1} ; m s_{2} ; \ldots, \sigma_{i-1}, s_{i}, i\right) \wedge \bigwedge_{j=1}^{\infty}\left(\alpha \vdash c s_{j} \sim_{t} m s_{j}\right) \wedge$

$\alpha \vdash c s \sim_{t} m s$

WHL, IF, (8)

(10) $\Longleftrightarrow P^{\prime}($ while $(e)\{c s\}, M)$

Case 1, (6)

DV , $(7,9)$

If there are break, return and continue in $c s$, the conclusions are also 
true. In a similar way, we can prove that

$$
\begin{aligned}
& (G, E \vdash e, M \Rightarrow \text { true }) \wedge\left(G, E \vdash c s, M \stackrel{t}{\Rightarrow} \text { Continue }, M_{1}\right) \wedge \\
& \left(G, E \vdash \text { while }(e)\{c s\}, M_{1} \stackrel{T}{\Rightarrow} \infty\right) \wedge P^{\prime}\left(\text { while }(e)\{c s\}, M_{1}\right) \\
\Longrightarrow & P^{\prime}(\text { while }(e)\{c s\}, M)
\end{aligned}
$$

6. For rules $D 8$ and $D 9$ w.r.t. " $x\left(e_{1}, \ldots, e_{m}\right) ; ", \operatorname{Stmt} \operatorname{Tr}\left(x\left(e_{1}, \ldots, e_{m}\right)\right.$; ) = " $f\left(r a_{1}, \ldots, r a_{m}, R V a l\right)$ ", where $r a_{k}=\operatorname{ExTr}\left(e_{k}\right)$ for all $1 \leq k \leq m$, if $x$ points to a user-defined function with a return value $\tau i d(p a r)\{d c l ; c s\}$, where $\operatorname{par}=\left(\tau_{1} y_{1}, \ldots, \tau_{m} y_{m}\right)$. Thus, $f$ points to function $i d\left(\tau_{1} y_{1}, \ldots, \tau_{m}\right.$ $\left.y_{m}, \tau R V a l\right)\{m d c l ; m s\}$ translated from $\tau i d(p a r)\{d c l ; c s\}$, where $m d c l=$ $\operatorname{DecTr}(d c l)$ and $m s=\operatorname{StmtTr}(\mathrm{cs})$.

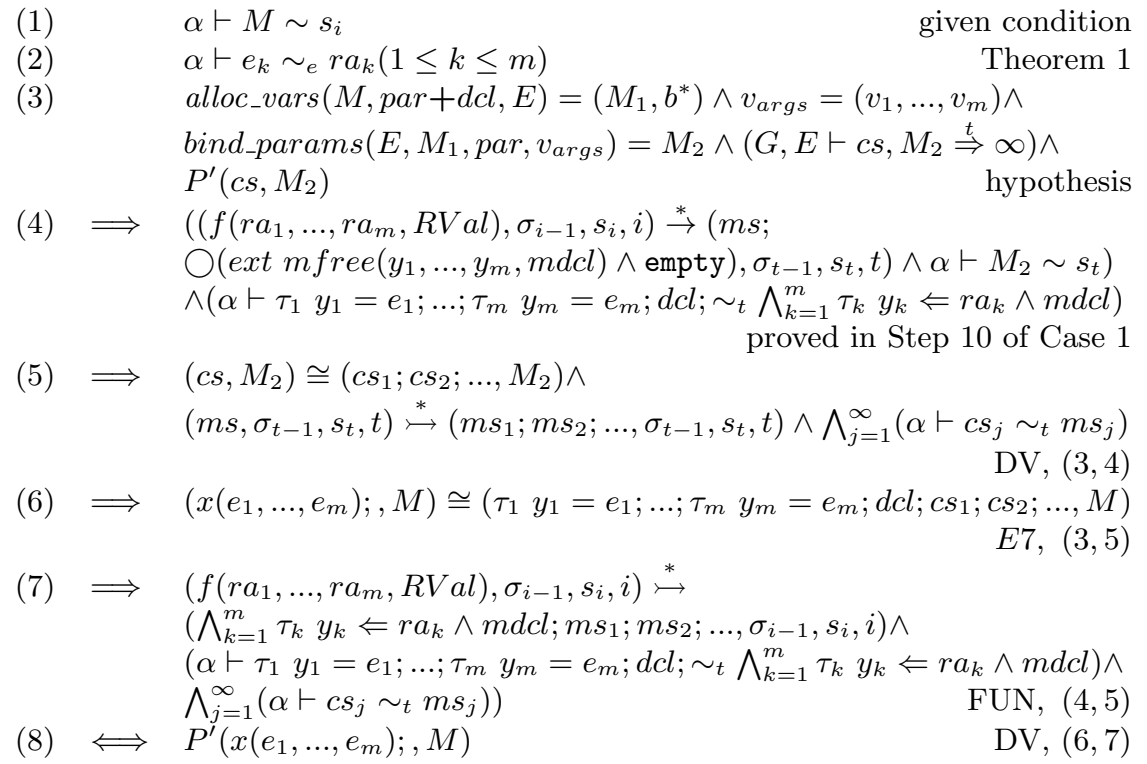

If $x$ points to a user-defined function without a return value, the conclusion can be proved in a similar way.

Based on the equivalences between expressions and statements in $\mathrm{Xd}-\mathrm{C}$ and MSVL, we have the following theorem:

Theorem 3. If an $X d$-C program $P$ is transformed to an $M S V L$ program $Q$ by Algorithm 4.1, then $P$ is semantically equivalent to $Q$, denoted by $P \sim_{p} Q$.

Proof: Suppose an Xd-C program $P$ is composed of $k_{1}$ expressions and $k_{2}$ statements, where $k_{1}$ and $k_{2}$ are constants. When $\mathrm{Xd}-\mathrm{C}$ program $P$ is transformed to MSVL program $Q$ by Algorithm 4.1, we have $Q=\operatorname{PrgmTr}(P)$. Actually, translating $P$ to $Q$ is merely translating expression $e_{i}\left(0 \leq i \leq k_{1}\right)$ and statement $c s_{j}\left(0 \leq j \leq k_{2}\right)$ in $P$ to $a_{i}$ and $m s_{j}$ in $Q$ in one-to-one manner by Algorithm 4.4 and 4.6 respectively. That is, $a_{i}=\operatorname{ExTr}\left(e_{i}\right)\left(0 \leq i \leq k_{1}\right)$ and 
$m s_{j}=\operatorname{Stmt} \operatorname{Tr}\left(c s_{j}\right)\left(0 \leq j \leq k_{2}\right)$. Let $M$ and $s_{0}$ be the initial states of $P$ and $Q$, respectively. According to Theorem 1 and 2 , for a given $\alpha$, if $\alpha \vdash M \sim s_{0}$, then $\alpha \vdash e_{i} \sim_{e} a_{i}$ and $\alpha \vdash c s_{j} \sim_{s} m s_{j}$ for all $0 \leq i \leq k_{1}$ and $0 \leq j \leq k_{2}$. As a result, $P$ is equivalent to $Q$, that is, $P \sim_{p} Q$.

\subsection{Time Complexity}

Let $t$ be the number of declarations, $n$ the number of statements in an Xd-C program and $m$ the average number of expressions in a statement. Correspondingly, it is not difficult to prove that the time complexity of the translation algorithm is $O(m \cdot n+t)$. Normally, the number of expressions in a statement is no more than a constant $k_{1}$ and the number of declarations in an Xd-C program is also no more than a constant $k_{2}$. As a result, the time complexity is $O(n)$.

\section{Implementation}

We have implemented the proposed approach in a tool named $C 2 M$. The architecture of the tool is shown in Fig. 3 An Xd-C program is first preprocessed. In this phase, \#include statements are removed by merging all Xd-C files in a project into a file according to their invoking relationships. Macro definitions such as \#ifdef, \#define and \#undef are processed using MinGW 33. to generate an Xd-C program without them. Then, lexer and parser of Xd-C programs based on Parser Generator (PG) are employed to do the lexical analysis and syntax analysis, respectively. Further, a syntax tree of an $\mathrm{Xd}-\mathrm{C}$ program is generated and translated to an MSVL program using the algorithms presented before. Finally, post processing adjusts the format of the generated MSVL program and outputs it to a file with a suffix of ".m". Since a generated MSVL program may invoke MSVL and Xd-C library functions, we have built our libraries of Xd-C and MSVL functions.

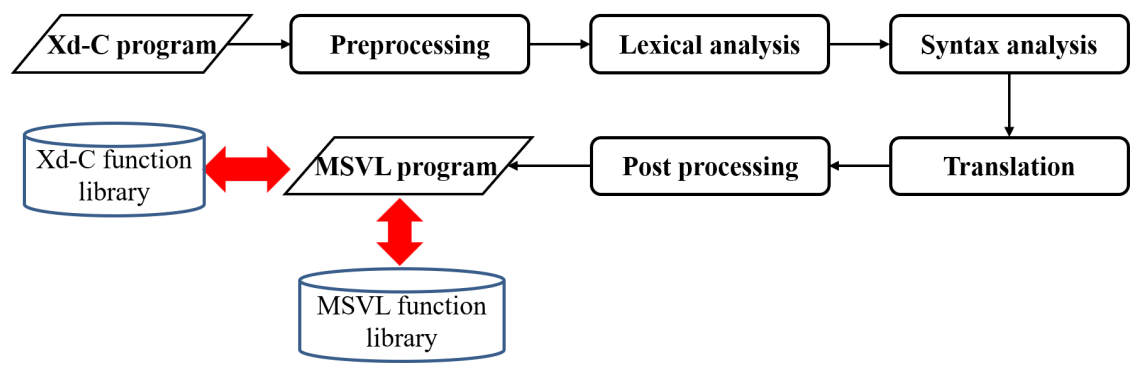

Figure 3: Architecture of $C 2 M$

In order to show the usability and scalability of our tool in translating realworld Xd-C programs to MSVL programs, we have applied $C 2 M$ on 13 programs from industry whose sizes range from $\approx 0.5 \mathrm{k}$ to $\approx 17 \mathrm{k}$ lines as shown in Table 7. In this benchmark, Xd-C programs from RERS P14 to RERS P19 are taken 
from RERS Grey-Box Challenge 2012 (RERS) [34]. LTLNFBA [35] is a software for translating an LTL formula to a Büchi automaton. Program carc [36] is a license plate recognition system. The other 5 programs bzip2, mcf, art, gzip and twolf are from SPEC2000 29. The experiments have been carried out on a 64-bit Windows $7 \mathrm{PC}$ with a $4.00 \mathrm{GHz}$ Intel(R) Core(TM) i7 processor and 64GB memory.

Table 7 shows the experimental results on the benchmark. Column "Program" represents names of programs. Column "LOC" shows sizes of Xd-C programs and column "LOM" lists sizes of MSVL programs translated from Xd-C programs. Column "Time" shows the time consumed for accomplishing the translation tasks. Experimental results in Table 7 show that for all the programs, our tool can effectively output the translation results and the size of the generated MSVL programs is about 2.6 times of Xd-C Programs.

Table 7: Results of C2M on real-world programs
\begin{tabular}{|c|c||c|c|}
\hline Program & LOC & LOM & Time $(\mathrm{s})$ \\
\hline \hline RERS P14 & 514 & 2261 & 0.46 \\
\hline RERS P15 & 1353 & 5016 & 2.04 \\
\hline RERS P16 & 1304 & 5271 & 2.18 \\
\hline RERS P17 & 2100 & 7753 & 4.38 \\
\hline RERS P18 & 3306 & 12677 & 11.81 \\
\hline RERS P19 & 8079 & 28332 & 63.83 \\
\hline LTLNFBA & 3296 & 9113 & 0.76 \\
\hline carc & 2170 & 4027 & 0.59 \\
\hline bzip2 & 2320 & 4976 & 0.55 \\
\hline mcf & 1322 & 2124 & 0.36 \\
\hline art & 886 & 1514 & 0.28 \\
\hline gzip & 3773 & 8189 & 0.80 \\
\hline twolf & 17452 & 33114 & 7.11 \\
\hline \hline Total & 47875 & 124376 & 95.15 \\
\hline
\end{tabular}

\section{Conclusion}

In order to verify safety, reliability and security properties of $\mathrm{C}$ programs by means of a runtime verification tool UMC4M [18, 19] based on MSVL and its compiler MC [23, we need to translate C programs to MSVL programs automatically. In this paper, we first present an approach to translating programs written in Xd-C to MSVL programs. Then we prove the equivalence between an original Xd-C program and the translated MSVL program. For doing so, we inductively prove the equivalences between expressions and statements, respectively involved in the Xd-C and MSVL programs. Further, we have developed a translator $C 2 M$ based on the proposed algorithms. Moreover, to evaluate usability and scalability of $C 2 M$, a benchmark of experiments including 13 programs from industry has been conducted. The results show that $C 2 M$ works 
effectively. However, since Xd-C is only a fragment of ANSI-C, to translate a general $\mathrm{C}$ program to an MSVL program, we have to manually translate the $\mathrm{C}$ program to an Xd-C program first.

In the near future, we plan to further optimize the translating approach and tool so as to improve efficiency of transformation. Further, we will build a library of functions for MSVL so that a large scale MSVL program can effectively be built. In addition, to verify properties of safety, reliability and security of $\mathrm{C}$ programs more effectively, we will further investigate techniques of runtime verification at code level by using translator $C 2 M$, MSVL compiler MC and verifier UMC4M of the toolkit MSV [37] such that C or MSVL programs in large scale can effectively be verified.

\section{References}

\section{References}

[1] E. M. Clarke, O. Grumberg, D. E. Long, Model checking and abstraction, ACM transactions on Programming Languages and Systems (TOPLAS) 16 (5) (1994) 1512-1542.

[2] E. M. Clarke, O. Grumberg, D. Peled, Model checking, MIT press, 1999.

[3] A. Cimatti, E. Clarke, E. Giunchiglia, F. Giunchiglia, M. Pistore, M. Roveri, R. Sebastiani, A. Tacchella, NuSMV 2: An opensource tool for symbolic model checking, in: Proceedings of the 14th International Conference on Computer Aided Verification (CAV), Vol. 2404 of Lecture Notes in Computer Science, Springer, 2002, pp. 359-364.

[4] G. J. Holzmann, The model checker SPIN, IEEE Transactions on Software Engineering 23 (5) (1997) 279-295.

[5] A. Pnueli, The temporal logic of programs, in: Proceedings of the 18th Annual Symposium on Foundations of Computer Science (FOCS), IEEE Computer Society, 1977, pp. 46-57.

[6] E. M. Clarke, E. A. Emerson, A. P. Sistla, Automatic verification of finitestate concurrent systems using temporal logic specifications, ACM Transactions on Programming Languages and Systems (TOPLAS) 8 (2) (1986) 244-263.

[7] M. Gheorghe, R. Ceterchi, F. Ipate, S. Konur, R. Lefticaru, Kernel p systems: From modelling to verification and testing, Theoretical Computer Science 724 (2018) $45-60$.

[8] A. Natarajan, H. Chauhan, N. Mittal, V. K. Garg, Efficient abstraction algorithms for predicate detection, Theoretical Computer Science 688 (2017) 24-48. 
[9] F. Ivancic, I. Shlyakhter, A. Gupta, M. K. Ganai, V. Kahlon, C. Wang, Z. Yang, Model checking C programs using F-SOFT, in: Proceedings of 23rd International Conference on Computer Design (ICCD), IEEE Computer Society, 2005, pp. 297-308.

[10] T. A. Henzinger, R. Jhala, R. Majumdar, G. Sutre, Lazy abstraction, in: Proceedings of the 29th ACM SIGPLAN-SIGACT Symposium on Principles of Programming Languages (POPL), ACM, 2002, pp. 58-70.

[11] D. Beyer, A. Cimatti, A. Griggio, M. E. Keremoglu, R. Sebastiani, Software model checking via large-block encoding, in: Proceedings of 9th International Conference on Formal Methods in Computer-Aided Design (FMCAD), IEEE, 2009, pp. 25-32.

[12] D. Dietsch, M. Heizmann, V. Langenfeld, A. Podelski, Fairness modulo theory: A new approach to LTL software model checking, in: Proceedings of the 27th International Conference on Computer Aided Verification (CAV), Vol. 9206 of Lecture Notes in Computer Science, Springer, 2015, pp. 49-66.

[13] T. Ball, S. K. Rajamani, Automatically validating temporal safety properties of interfaces, in: Proceedings of the 8th international SPIN workshop on Model checking of software, Vol. 2057 of Lecture Notes in Computer Science, Springer, 2001, pp. 103-122.

[14] D. Beyer, T. A. Henzinger, R. Jhala, R. Majumdar, The software model checker BLAST: Applications to software engineering, International Journal on Software Tools for Technology Transfer 9 (5) (2007) 505-525.

[15] D. Beyer, M. E. Keremoglu, CPAchecker: A tool for configurable software verification, in: Proceedings of 23rd International Conference on Computer Aided Verification (CAV), Vol. 6806 of Lecture Notes in Computer Science, Springer, 2011, pp. 184-190.

[16] D. Kroening, M. Tautschnig, CBMC - C bounded model checker, in: Proceedings of 20th International Conference on Tools and Algorithms for the Construction and Analysis of Systems (TACAS), Vol. 8413 of Lecture Notes in Computer Science, Springer, 2014, pp. 389-391.

[17] M. Brockschmidt, B. Cook, S. Ishtiaq, H. Khlaaf, N. Piterman, T2: temporal property verification, in: Proceedings of the 22nd International Conference on Tools and Algorithms for the Construction and Analysis of Systems (TACAS), Vol. 9636 of Lecture Notes in Computer Science, Springer, 2016, pp. $387-393$.

[18] M. Wang, C. Tian, Z. Duan, Full regular temporal property verification as dynamic program execution, in: Proceedings of the 39th International Conference on Software Engineering (ICSE) - Companion Volume, IEEE Computer Society, 2017, pp. 226-228. 
[19] M. Wang, C. Tian, N. Zhang, Z. Duan, Verifying full regular temporal properties of programs via dynamic program execution, IEEE Transactions on Reliability (2018) 1-16doi:10.1109/TR.2018.2876333.

[20] Z. Duan, An extended interval temporal logic and a framing technique for temporal logic programming, Ph.D. thesis, Department of Computer Science, Newcastle University, Newcastle upon Tyne, UK (1996).

[21] Z. Duan, Temporal logic and temporal logic programming, Alexandria, NSW, Australia: Science Press, 2005.

[22] N. Zhang, Z. Duan, C. Tian, A mechanism of function calls in MSVL, Theoretical Computer Science 654 (2016) 11-25.

[23] K. Yang, Z. Duan, C. Tian, N. Zhang, A compiler for MSVL and its applications, Theoretical Computer Science 749 (2018) 2-16.

[24] Z. Duan, C. Tian, A practical decision procedure for propositional projection temporal logic with infinite models, Theoretical Computer Science 554 (2014) 169-190.

[25] Z. Duan, C. Tian, L. Zhang, A decision procedure for propositional projection temporal logic with infinite models, Acta Informatica 45 (1) (2008) 43-78.

[26] S. Blazy, X. Leroy, Mechanized semantics for the Clight subset of the C language, Journal of Automated Reasoning 43 (3) (2009) 263-288.

[27] X. Yang, Z. Duan, Operational semantics of framed tempura, The Journal of Logic and Algebraic Programming 78 (1) (2008) 22-51.

[28] X. Wang, C. Tian, Z. Duan, L. Zhao, MSVL: a typed language for temporal logic programming, Frontiers of Computer Science 11 (5) (2017) 762-785.

[29] Standard Performance Evaluation Corporation. SPEC CINT2000, October 2008., http://www.spec.org/cpu/CINT2000/.

[30] Z. Duan, X. Yang, M. Koutny, Framed temporal logic programming, Science of Computer Programming 70 (1) (2008) 31-61.

[31] Z. Duan, M. Koutny, A framed temporal logic programming language, Journal of Computer Science and Technology 19 (3) (2004) 341-351.

[32] S. Blazy, Z. Dargaye, X. Leroy, Formal verification of a C compiler frontend, in: Proceedings of the 14th International Symposium on Formal Methods (FM), Vol. 4085 of Lecture Notes in Computer Science, Springer, 2006, pp. $460-475$.

[33] http://www.mingw.org/ (2018). 
[34] F. Howar, M. Isberner, M. Merten, B. Steffen, D. Beyer, The RERS greybox challenge 2012: Analysis of event-condition-action systems, in: Proceedings of the 5th International Symposium on Leveraging Applications of Formal Methods, Verification and Validation (ISoLA), Vol. 7609 of Lecture Notes in Computer Science, Springer, 2012, pp. 608-614.

[35] http://web.xidian.edu.cn/ctian/en/ltlnfba.html (2018).

[36] http://www. codeforge.cn/read/234151/car.c__html (2014).

[37] N. Zhang, Z. Duan, C. Tian, Model checking concurrent systems with MSVL, SCIENCE CHINA Information Sciences 59 (11) (2016) 118101.

\section{Appendix}

Appendix A: Operational Semantics of Xd-C

The following is operational semantics of expressions in Xd-C.

Expressions in left-value position:

$$
\begin{aligned}
& \frac{E(i d)=b \text { or }(i d \notin D o m(E) \text { and symbol }(G, i d)=\lfloor b\rfloor)}{G, E \vdash i d, M \stackrel{l}{\Rightarrow}(b, 0)}(C 1) \quad \frac{G, E \vdash e, M \Rightarrow p \operatorname{tr}(\ell)}{G, E \vdash * e, M \stackrel{l}{\Rightarrow} \ell}(C 2) \\
& \frac{G, E \vdash l e, M \stackrel{l}{\Rightarrow}(b, \delta) \quad \text { type }(l e)=\text { struct } i d^{\prime}\{\varphi\} \quad \text { field_offset }(x, \varphi)=\left\lfloor\delta^{\prime}\right\rfloor}{G, E \vdash \text { le.x, } M \stackrel{l}{\Rightarrow}\left(b, \delta+\delta^{\prime}\right)}(C 3)
\end{aligned}
$$

where $\operatorname{Dom}(E)$ is the domain of $E ; p \operatorname{tr}(\ell)$ is a pointer value pointing to $\ell ;$ type $(e)$ returns the type of $e$; struct $i d^{\prime}\{\varphi\}$ is a struct type where $i d^{\prime}$ is the name and a list $\varphi$ is its fields; field_offset $(x, \varphi)$ returns the byte offset of the field named $x$ in a struct whose field list is $\varphi$.

In order to access and store values in memory locations, $\operatorname{loadval}(\tau, M,(b, \delta))$ 
and $\operatorname{storeval}(\tau, M,(b, \delta), v)$ are respectively defined as follows:

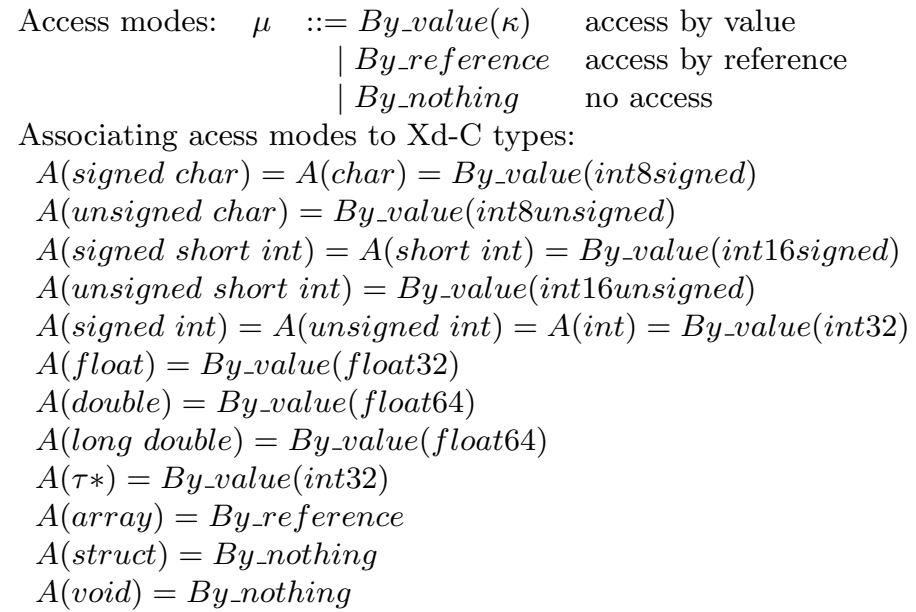

Accessing or updating a value of type $\tau$ at location $(b, \delta)$ in memory state $M$ :

$$
\begin{aligned}
\operatorname{loadval}(\tau, M,(b, \delta)) & =\operatorname{load}(\kappa, M, b, \delta) & & \text { if } A(\tau)=\text { By_value }(\kappa) \\
\operatorname{loadval}(\tau, M,(b, \delta)) & =\lfloor\operatorname{tg}(b, \delta)\rfloor & & \text { if } A(\tau)=\text { By_reference } \\
\text { loadval }(\tau, M,(b, \delta)) & =\emptyset & & \text { if } A(\tau)=\text { By_nothing } \\
\operatorname{storeval}(\tau, M,(b, \delta), v) & =\operatorname{store}(\kappa, M, b, \delta, v) & & \text { if } A(\tau)=\text { By_value }(\kappa) \\
\operatorname{storeval}(\tau, M,(b, \delta), v) & =\emptyset & & \text { otherwise }
\end{aligned}
$$

loadval $(\tau, M,(b, \delta))$ reads consecutive bytes at $(b, \delta)$ in memory state $M$ and returns the contents of these bytes as value $v$ if successful while $\operatorname{storeval}(\tau, M,(b, \delta)$, $v)$ stores value $v$ at $(b, \delta)$ in memory state $M$ and returns an updated memory state.

Expressions in right-value position:

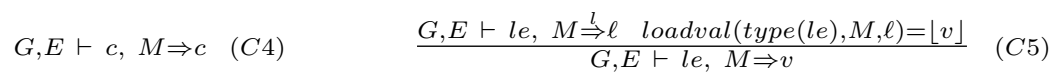

$$
\begin{aligned}
& \frac{G, E \vdash l e, M \stackrel{l}{\Rightarrow} \ell}{G, E \vdash \& l e, M \Rightarrow p \operatorname{tr}(\ell)}(C 6) \quad \frac{G, E \vdash e_{1}, M \Rightarrow v_{1} \text { eval_unop }\left(o p_{1}, v_{1}, \text { type }\left(e_{1}\right)\right)=\lfloor v\rfloor}{G, E \vdash o p_{1} e_{1}, M \Rightarrow v}(C 7) \\
& \frac{G, E \vdash e_{1}, M \Rightarrow v_{1} \quad G, E \vdash e_{2}, M \Rightarrow v_{2} \quad \text { eval_binop }\left(o p_{2}, v_{1}, \text { type }\left(e_{1}\right), v_{2}, \text { type }\left(e_{2}\right)\right)=\lfloor v\rfloor}{G, E \vdash e_{1} \quad o p_{2} \quad e_{2}, M \Rightarrow v}(C 8) \\
& \frac{G, E \vdash e_{1}, M \Rightarrow v_{1} \quad i s_{t} \operatorname{true}\left(v_{1}, \operatorname{type}\left(e_{1}\right)\right) \quad G, E \vdash e_{2}, M \Rightarrow v_{2}}{G, E \vdash e_{1} ? e_{2}: e_{3}, M \Rightarrow v_{2}}(C 9) \\
& \frac{G, E \vdash e_{1}, M \Rightarrow v_{1} \quad i s_{f} \text { false }\left(v_{1}, \text { type }\left(e_{1}\right)\right) \quad G, E \vdash e_{3}, M \Rightarrow v_{3}}{G, E \vdash e_{1} ? e_{2}: e_{3}, M \Rightarrow v_{3}}(C 10) \\
& \frac{G, E \vdash e, M \Rightarrow v_{1} \quad \operatorname{cast}\left(v_{1}, \operatorname{type}(e), \tau\right)=\lfloor v\rfloor}{G, E \vdash(\tau) e, M \Rightarrow v}(C 11) \\
& \frac{G, E \vdash e_{\text {fun }}\left(e_{\text {args }}\right), M \stackrel{t}{\Rightarrow} v, M}{G, E \vdash e_{\text {fun }}\left(e_{\text {args }}\right), M \Rightarrow v}(C 12)
\end{aligned}
$$

where eval_unop $\left(o p_{1}, v_{1}, \operatorname{type}\left(e_{1}\right)\right)$ describes a unary operation and returns the value of $o p_{1} v_{1}$; eval_binop $\left(o p_{2}, v_{1}\right.$, type $\left(e_{1}\right), v_{2}$, type $\left.\left(e_{2}\right)\right)$ returns the value of $v_{1} o_{2} v_{2} ;$ is_true $\left(v_{1}, \operatorname{type}\left(e_{1}\right)\right)$ and $i s_{-} f a l s e\left(v_{1}\right.$, type $\left.\left(e_{1}\right)\right)$ determine the truth value of $v_{1}$, depending on its type, and the truth value of $v_{1}$ is false if $v_{1}$ equals 0 and true otherwise; $\operatorname{cast}\left(v_{1}\right.$, type $\left.(e), \tau\right)$ converts $v_{1}$ from its natural type type $(e)$ to the expected type $\tau$. 
Table 8: Binary addition

\begin{tabular}{lllll}
\hline$\tau_{1}$ & $\tau_{2}$ & $v_{1}$ & $v_{2}$ & eval_binop $\left(+, v_{1}, \tau_{1}, v_{2}, \tau_{2}\right)$ \\
\hline int & int & $n_{1}$ & $n_{2}$ & $n_{1}+n_{2}$ \\
float & float & $f_{1}$ & $f_{2}$ & $f_{1}+f_{2}$ \\
double & double & $d_{1}$ & $d_{2}$ & $d_{1}+d_{2}$ \\
$\tau *$ & int & $p \operatorname{tr}(b, \delta)$ & $n$ & $\operatorname{ptr}(b, \delta+n * \operatorname{sizeof}(\tau))$ \\
int & $\tau *$ & $n$ & $p \operatorname{tr}(b, \delta)$ & $\operatorname{ptr}(b, \delta+n * \operatorname{sizeof}(\tau))$ \\
& \multicolumn{3}{c}{ otherwise } & $\emptyset$ \\
\hline
\end{tabular}

Taking binary addition as an example, the two argument expressions of types $\tau_{1}$ and $\tau_{2}$ are evaluated and their values $v_{1}$ and $v_{2}$ are combined using the the eval_binop function. The cases corresponding to binary addition are shown in Table 8 ,

Some forms of $\mathrm{C}$ expressions are omitted but can be expressed as syntactic sugar:

array access: $\quad i d[e] \equiv *(i d+e) \quad C 13$

$i d\left[e_{1}\right]\left[e_{2}\right] \equiv *\left(i d+e_{1} * n+e_{2}\right)$, where $n$ is the number of elements in each row of $i d\left[e_{1}\right]\left[e_{2}\right] . \quad C 14$

indirect field access: $\quad e \rightarrow x \equiv *(e . x) \quad C 15$

Operational semantics for Xd-C statements (other than loops and switch statements):

$$
\begin{aligned}
& G, E \vdash ;, M \stackrel{\epsilon}{\Rightarrow} \text { Normal, } M(T 1) \quad G, E \vdash \text { break;, } M \stackrel{\epsilon}{\Rightarrow} \text { Break, } M \quad(T 2) \\
& G, E \vdash \text { continue; } M \stackrel{\epsilon}{\Rightarrow} \text { Continue, } M(T 3) \quad G, E \vdash \text { return;, } M \stackrel{\epsilon}{\Rightarrow} \text { Return, } M \\
& G, E \vdash e, M \Rightarrow v, M^{\prime} \\
& \overline{G, E \vdash \text { return } e ;, M \stackrel{\epsilon}{\Rightarrow} \operatorname{Return}(v), M^{\prime}} \\
& \frac{G, E \vdash l e, M \stackrel{l}{\Rightarrow} \ell \quad G, E \vdash e, M \Rightarrow v \quad \text { storeval }(\text { type }(l e), M, \ell, v)=\left\lfloor M^{\prime}\right\rfloor}{G, E \vdash l e=e, M \stackrel{\epsilon}{\Rightarrow} \text { Normal, } M^{\prime}}(T 6)
\end{aligned}
$$

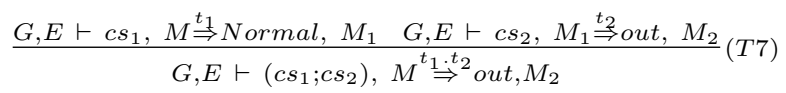

$$
\begin{aligned}
& \frac{G, E \vdash c s_{1}, M \stackrel{t}{\Rightarrow} \text { out }, M^{\prime} \text { out } \neq \text { Normal }}{G, E \vdash\left(c s_{1} ; c s_{2}\right), M \stackrel{t}{\Rightarrow} \text { out }, M^{\prime}}(T 8) \\
& \frac{G, E \vdash e, M \Rightarrow v \quad \text { is_true }(v, \text { type }(e)) \quad G, E \vdash c s_{1}, M \stackrel{t}{\Rightarrow} \text { out }, M^{\prime}}{G, E \vdash \text { if }(e)\left\{c s_{1}\right\} \operatorname{else}\left\{c s_{2}\right\}, M \stackrel{t}{\Rightarrow} \text { out }, M^{\prime}} \\
& \frac{G, E \vdash e, M \Rightarrow v \quad \text { is_false }(v, \text { type }(e)) \quad G, E \vdash c s_{2}, M \stackrel{t}{\Rightarrow} \text { out }, M^{\prime}}{G, E \vdash \operatorname{if}(e)\left\{c s_{1}\right\} \operatorname{else}\left\{c s_{2}\right\}, M \stackrel{t}{\Rightarrow} \text { out }, M^{\prime}}
\end{aligned}
$$

$l e++$ and $l e--$ are omitted but can be expressed as follows:

$$
\begin{aligned}
& l e++\equiv(l e=l e+1) \\
& l e--\equiv(l e=l e-1)
\end{aligned}
$$

The following rules define the execution of while and for loops. The rules describing the execution of do loops resemble the rules for while loops and are 
omitted in this paper.

Outcome updates (at the end of a loop execution):

$$
\text { Break } \stackrel{\text { loop }}{\rightsquigarrow} \text { Normal Return } \stackrel{\text { loop }}{\rightsquigarrow} \operatorname{Return} \quad \operatorname{Return}(v) \stackrel{\text { loop }}{\rightsquigarrow} \operatorname{Return}(v)
$$

while loops:

$$
\begin{aligned}
& \underline{G, E \vdash e, M \Rightarrow v \quad \text { is_false }(v, \text { type }(e))} \\
& G, E \vdash \text { while }(e)\{c s\}, M \stackrel{\epsilon}{\Rightarrow} \text { Normal, } M \quad(T 11) \\
& \frac{G, E \vdash e, M \Rightarrow v \quad \text { is_true }(v, \text { type }(e)) \quad G, E \vdash c s, M \stackrel{t}{\Rightarrow} \text { out }, M^{\prime} \quad \text { out } \stackrel{\text { loop }}{\rightsquigarrow} \text { out }}{} \\
& G, E \vdash e, M \Rightarrow v \quad \text { is_true(v,type(e)) } \\
& G, E \vdash c s, M \stackrel{t_{1}}{\Rightarrow}(\text { Normal } \mid \text { Continue }), M_{1} \quad G, E \vdash \text { while }(e)\{c s\}, M_{1} \stackrel{t_{2}}{\Rightarrow} \text { out }{ }^{\prime}, M_{2} \\
& G, E \vdash \text { while }(e)\{c s\}, M^{t_{1} \cdot t_{2}} \stackrel{o^{\prime}}{\Rightarrow} t^{\prime}, M_{2}
\end{aligned}
$$

for loops:

$$
\begin{aligned}
& \underline{c s_{1} \neq ; \quad G, E \vdash c s_{1}, M \stackrel{t_{1}}{\Rightarrow} \text { Normal, } M_{1} \quad G, E \vdash \text { for }\left(; e ; c s_{2}\right)\{c s\}, M_{1} \stackrel{t_{2}}{\Rightarrow} \text { out }, M_{2}} \\
& G, E \vdash \text { for }\left(c s_{1} ; e ; c s_{2}\right)\{c s\}, M^{t_{1} . t_{2}} \stackrel{\text { out }, M_{2}}{\Rightarrow} \\
& G, E \vdash e, M \Rightarrow v \quad \text { is_false }(v, \text { type }(e)) \\
& \overline{G, E \vdash \text { for }\left(; e ; c s_{2}\right)\{c s\}, M \stackrel{\epsilon}{\Rightarrow} \text { Normal, } M}(T 15) \\
& \frac{G, E \vdash e, M \Rightarrow v \quad \text { is_true }(v, \text { type }(e)) \quad G, E \vdash c s, M \stackrel{t}{\Rightarrow} \text { out }_{1}, M^{\prime} \quad \text { out }_{1} \stackrel{\text { loop }}{\rightsquigarrow} \text { out }}{} \\
& G, E \vdash e, M \Rightarrow v \quad i s_{-} \operatorname{true}(v, \operatorname{type}(e)) \quad G, E \vdash c s, M \stackrel{t_{1}}{\Rightarrow}(\text { Normal } \mid \text { Continue }), M_{1} \\
& G, E \vdash c s_{2}, M_{1} \stackrel{t_{2}}{\Rightarrow} N \text { ormal }, M_{2} \quad G, E \vdash \text { for }\left(; e ; c s_{2}\right)\{c s\}, M_{2} \stackrel{t_{3}}{\Rightarrow} \text { out }, M_{3} \\
& G, E \vdash \text { for }\left(; e ; c s_{2}\right)\{c s\}, M^{t_{1}} \stackrel{t_{2} \cdot t_{3}}{\Longrightarrow} \text { out }, M_{3}
\end{aligned}
$$

Outcome updates (at the end of a switch execution):

$$
\begin{aligned}
& \text { Normal } \underset{\sim}{\text { switch }} \text { Normal Continue } \stackrel{\text { switch }}{\rightsquigarrow} \text { Continue Break } \stackrel{\text { switch }}{\rightsquigarrow} \text { Normal } \\
& \text { Return } \stackrel{\text { switch }}{\rightsquigarrow} \operatorname{Return} \quad \operatorname{Return}(v) \stackrel{\text { switch }}{\rightsquigarrow} \operatorname{Return}(v) \\
& \text { switch }(e)\left\{\text { case } n_{0}: c s_{0} ; \ldots ; \text { case } n_{m}: c s_{m} ; \operatorname{def} \text { ault: } c s ;\right\} \text { : } \\
& \frac{G, E \vdash c s, M \stackrel{t}{\Rightarrow} \text { out }, M^{\prime}}{G, E \vdash \operatorname{default}: c s ;, M \stackrel{t}{\Rightarrow} \text { out }, M^{\prime}} \quad(T 18) \quad \frac{G, E \vdash c s, M \stackrel{t}{\Rightarrow} \text { out }, M^{\prime}}{G, E \vdash \text { case }: c s ;, M \stackrel{t}{\Rightarrow} \text { out }, M^{\prime}} \\
& G, E \vdash \text { case } n_{i}: c s_{i} ;, M \stackrel{t_{1}}{\Rightarrow} \text { Normal, } M_{1} \\
& \frac{G, E \vdash \text { case } n_{i+1}: c s_{i+1} ; \ldots ; \text { case } n_{m}: c s_{m} ; \text { defalut: } c s ;, M \stackrel{t_{2}}{\Rightarrow} \text { out, } M_{2}}{G, E \vdash \text { case } n_{i}: c s_{i} ; \ldots ; \text { case } n_{m}: c s_{m} ; \text { defalut: } c s ;, M^{t_{1} \cdot t_{2}} \Rightarrow \text { out, } M_{2}}(T 20) \\
& G, E \vdash \text { case } n_{i}: c s_{i} ;, M \stackrel{t}{\Rightarrow} \text { out, } M^{\prime} \text { out } \neq \text { Normal } \\
& \overline{G, E \vdash \text { case } n_{i}: c s_{i} ; \ldots ; \text { case } n_{m}: c s_{m} ; \text { defalut: } c s ;, M \stackrel{t}{\Rightarrow} \text { out }, M^{\prime}}(T 21) \\
& G, E \vdash e, M \Rightarrow v \quad v==n_{i} \\
& \frac{G, E \vdash \text { case } n_{i}: c s_{i} ; \ldots ; \text { case } n_{m}: c s_{m} ; \text { defalut: } c s ;, M \stackrel{t}{\Rightarrow} \text { out }_{1}, M^{\prime} \quad \text { out }_{1} \stackrel{\text { switch }}{\sim} \text { out }}{G, E \vdash \operatorname{switch}(e)\left\{\text { case } n_{0}: c s_{0} ; \ldots ; \text { case } n_{m}: c s_{m} ; \text { defalut: } c s ;\right\}, M \stackrel{t}{\Rightarrow} \text { out }, M^{\prime}}(T 22) \\
& G, E \vdash e, M \Rightarrow v \quad v !=n_{0} \quad v !=n_{1} \quad \ldots \quad v !=n_{m} \\
& G, E \vdash \text { defalut: } c s ;, M \stackrel{t}{\Rightarrow} \text { out }_{1}, M^{\prime} \quad \text { out }_{1} \stackrel{\text { switch }}{\leftrightarrow} \text { out } \\
& \overline{G, E \vdash \operatorname{switch}(e)\left\{\text { case } n_{0}: c s_{0} ; \ldots ; \text { case } n_{m}: c s_{m} ; \text { defalut: } c s ;\right\}, M \stackrel{t}{\Rightarrow} \text { out }, M^{\prime}}(T 23)
\end{aligned}
$$


Function calls:

$$
\begin{gathered}
G, E \vdash e_{\text {fun }}, M \Rightarrow p \operatorname{tr}(b, 0) \quad G, E \vdash e_{\text {args }}, M \Rightarrow v_{\text {args }} \\
\text { functdef }(G, b)=\lfloor\text { funct }\rfloor \quad \text { type_of } f \text { fundef }(\text { funct })=\text { type }\left(e_{\text {fun }}\right) \\
G \vdash \text { funct }\left(v_{\text {args }}\right), M \stackrel{t}{\Rightarrow} v_{\text {res }}, M^{\prime} \\
G, E \vdash e_{\text {fun }}\left(e_{\text {args }}\right), M \stackrel{t}{\Rightarrow} v_{\text {res }}, M^{\prime}
\end{gathered}
$$

where $e_{f u n}$ is a function pointer or a function name, $e_{\text {args }}$ a list of arguments of the function and $v_{\text {args }}$ a list of values of arguments; type_of_fundef (funct) returns the type of function funct including the return type and types of parameters; $v_{\text {res }}$ is the return value of funct $\left(v_{\text {args }}\right)$.

Compatibility between values, outcomes and return types:

$$
\text { Normal,void\#undef } \quad \text { Return,void\#undef } \quad \operatorname{Return}(v), \tau \# v \text { when } \tau \neq \text { void }
$$

Function invocations:

$$
\begin{aligned}
& \text { funct }=[\tau \mid \text { void }] \text { id }(\text { par })\{d c l ; c s\} \quad \text { alloc_vars }(M, p a r+d c l, E)=\left(M_{1}, b^{*}\right) \\
& \frac{\text { bind_params }\left(E, M_{1}, \text { par, } v_{\text {args }}\right)=M_{2} \quad G, E \vdash c s, M_{2} \stackrel{t}{\Rightarrow} \text { out }, M_{3} \quad \text { out, } \tau \# v_{\text {res }}}{G \vdash \operatorname{funct}\left(v_{\text {args }}\right), M \stackrel{t}{\Rightarrow} v_{\text {res }}, \text { free }\left(M_{3}, b^{*}\right)} \text { (T25) }
\end{aligned}
$$

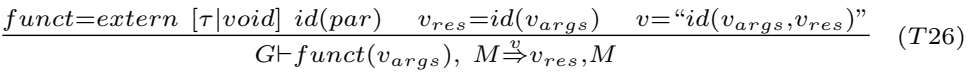

where $d c l$ is a list of declarations $\left(d c l=(P d ;)^{*}\right)$; alloc_vars $(M, \operatorname{par}+d c l, E)$ allocates the memory required for storing the formal parameters par and the local variables $d c l$; bind_params $\left(E, M_{1}\right.$, par, $\left.v_{\text {args }}\right)$ iterates the storeval function in order to initialize formal parameters par to the values of the corresponding arguments $v_{\text {args }} ; v_{\text {res }}=i d\left(v_{\text {args }}\right)$ obtains the return value of the function call $i d\left(v_{\text {args }}\right)$ and if the return type is void, the value of $v$ is $\emptyset$. An input/output event $v$ recorded in the trace is generated by a call to an external function. 
Operational semantics for divergence:

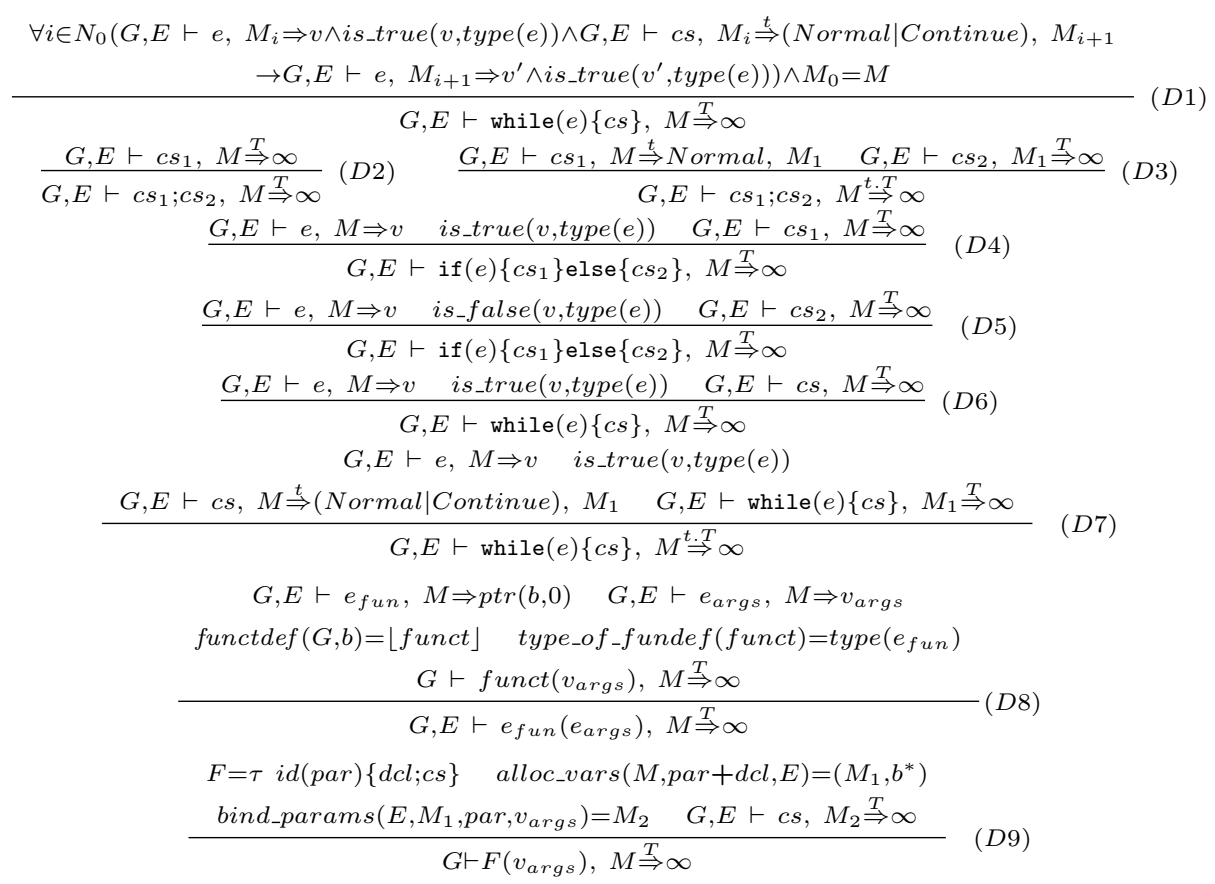

Observable behaviors of programs:

$$
\begin{aligned}
& G=\text { globalenv }(P) \quad M=\text { initme }(P) \\
& \operatorname{symbol}(G, \operatorname{main}(P))=\lfloor b\rfloor \quad \text { functdef }(G, b)=\lfloor f\rfloor \quad(f(\text { nil }), G, E, M) \stackrel{t}{\Rightarrow} n, M^{\prime} \\
& \vdash P \Rightarrow \operatorname{terminates}(t, n) \\
& G=\text { globalenv }(P) \quad M=\text { initmem }(P) \\
& \frac{\operatorname{symbol}(G, \operatorname{main}(P))=\lfloor b\rfloor \quad \text { functdef }(G, b)=\lfloor f\rfloor \quad G, E \vdash f(n i l), M \stackrel{T}{\Rightarrow} \infty}{\vdash P \Rightarrow \operatorname{diverges}(T)}
\end{aligned}
$$

A global environment $G$ and a memory state $M$ are computed for $P$. If the main function invocation terminates with trace $t$ and result value $n$, the observed behavior of $\mathrm{P}$ is terminates $(t, n)$. If the function invocation diverges with a possibly infinite trace $T$, the observed behavior is diverges $(T)$.

Based on the operational semantics, some semantic equivalence rules can be 
proved similarly to Lemma 2 and are given as follows:

$$
\begin{aligned}
& E 1 \quad(c s, M) \cong\left(c s_{1} ; c s_{2}, M\right) \\
& \Longrightarrow\left(c s ; c s^{\prime}, M\right) \cong\left(c s_{1} ; c s_{2} ; c s^{\prime}, M\right) \\
& E 2 \quad\left(c s^{\prime}, M^{\prime}\right) \cong\left(c s_{1} ; c s_{2}, M^{\prime}\right) \wedge\left(G, E \vdash c s, M \stackrel{t}{\Rightarrow} \text { out }, M^{\prime}\right) \\
& \Longrightarrow\left(c s ; c s^{\prime}, M\right) \cong\left(c s ; c s_{1} ; c s_{2}, M\right) \\
& \text { E3 } G, E \vdash e, M \Rightarrow \text { true } \\
& \Longrightarrow\left(\mathrm{if}(e)\{c s\} \mathrm{else}\left\{c s^{\prime}\right\}, M\right) \cong(c s, M) \\
& \text { E4 } G, E \vdash e, M \Rightarrow \text { false } \\
& \Longrightarrow\left(\operatorname{if}(e)\{c s\} \text { else }\left\{c s^{\prime}\right\}, M\right) \cong\left(c s^{\prime}, M\right) \\
& \text { E5 }(G, E \vdash e, M \Rightarrow \text { true }) \wedge\left(G, E \vdash c s, M \stackrel{t}{\Rightarrow}(\text { Normal } \mid \text { Continue }), M_{1}\right) \\
& \Longrightarrow(\operatorname{while}(e)\{c s\}, M) \cong(c s ; \operatorname{while}(e)\{c s\}, M) \\
& \text { E6 } \quad(G, E \vdash e, M \Rightarrow \text { true }) \wedge(G, E \vdash c s, M \stackrel{T}{\Rightarrow} \infty) \\
& \Longrightarrow(\text { while }(e)\{c s\}, M) \cong(c s, M) \\
& \text { E7 }\left(G, E \vdash e_{\text {fun }}, M \Rightarrow \operatorname{ptr}(b, 0)\right) \wedge \operatorname{functdef}(G, b)=\lfloor\text { funct }\rfloor \wedge \\
& \text { type_of_funde } f(\text { funct })=\text { type }\left(e_{\text {fun }}\right) \wedge \\
& \text { funct }=[\tau \mid \text { void }] i d\left(\tau_{1} y_{1}, \ldots, \tau_{m} y_{m}\right)\{d c l ; c s\} \wedge(G, E \vdash c s, M \stackrel{T}{\Rightarrow} \infty) \\
& \Longrightarrow\left(e_{\text {fun }}\left(e_{1}, \ldots, e_{m}\right), M\right) \cong\left(\tau_{1} y_{1}=e_{1} ; \ldots ; \tau_{m} y_{m}=e_{m} ; d c l ; c s, M\right)
\end{aligned}
$$

Appendix B: Semantics of MSVL statements

Table 9 shows that all MSVL statements are defined by PTL formulas.

Table 9: Syntax and Semantics of MSVL statements

\begin{tabular}{ll}
\hline SyntaX & SEMANTICS \\
\hline empty & $\stackrel{\text { def }}{=} \varepsilon$ \\
skip & $\stackrel{\text { def }}{=} \bigcirc \varepsilon$ \\
$l a \Leftarrow r a$ & $\stackrel{\text { def }}{=} l a=r a \wedge p_{l a}$ \\
$l a:=r a$ & $\stackrel{\text { def }}{=} \bigcirc\left(l a=r a \wedge p_{l a}\right) \wedge \bigcirc \varepsilon$ \\
$m s_{1}$ and $m s_{2}$ & $\stackrel{\text { def }}{=} m s_{1} \wedge m s_{1}$ \\
next $m s$ & $\stackrel{\text { def }}{=} \bigcirc m s$ \\
$m s_{1} ; m s_{2}$ & $\stackrel{\text { def }}{=} m s_{1} ; m s_{2}$ \\
if $(b) \operatorname{then}\left\{m s_{1}\right\} \operatorname{else}\left\{m s_{2}\right\}$ & $\stackrel{\text { def }}{=}\left(b \rightarrow m s_{1}\right) \wedge\left(\neg b \rightarrow m s_{2}\right)$ \\
while $(b)\{m s\}$ & $\stackrel{\text { def }}{=}(b \wedge m s)^{*} \wedge \square(\varepsilon \rightarrow \neg b) \vee(b \wedge m s)^{\omega}$ \\
\hline
\end{tabular}

